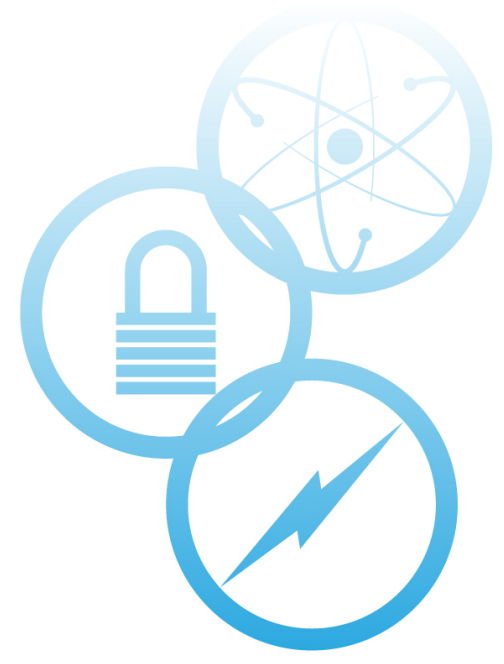

\title{
Multiscale-Informed Modeling of High Temperature Component Response with Uncertainty Quantification
}

\section{August 2020}

Idaho National Laboratory

L. B. Munday

S. L. Dhulipala

A. Casagranda

S. A. Pitts

B. W. Spencer

Los Alamos National Laboratory

L. Capolungo

A. E. Tallman

M. Arul Kumar

C. Matthews

Argonne National Laboratory

M.C. Messner

A. E. Chakraborty

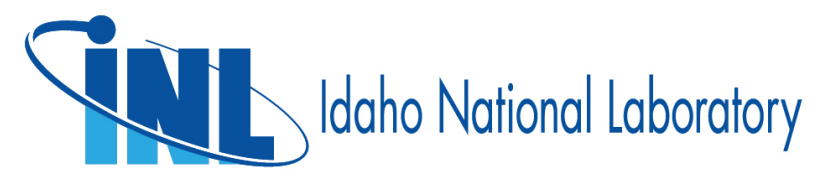




\section{DISCLAIMER}

This information was prepared as an account of work sponsored by an agency of the U.S. Government. Neither the U.S. Government nor any agency thereof, nor any of their employees, makes any warranty, expressed or implied, or assumes any legal liability or responsibility for the accuracy, completeness, or usefulness, of any information, apparatus, product, or process disclosed, or represents that its use would not infringe privately owned rights. References herein to any specific commercial product, process, or service by trade name, trade mark, manufacturer, or otherwise, does not necessarily constitute or imply its endorsement, recommendation, or favoring by the U.S. Government or any agency thereof. The views and opinions of authors expressed herein do not necessarily state or reflect those of the U.S. Government or any agency thereof. 


\section{Multiscale-Informed Modeling of High Temperature Component Response with Uncertainty Quantification}

L. B. Munday ${ }^{1}$

S. L. Dhulipala ${ }^{1}$

S. A. Pitts ${ }^{1}$

A. Casagranda ${ }^{1}$

B. W. Spencer ${ }^{1}$

L. Capolungo ${ }^{2}$

A. E. Tallman ${ }^{2}$

M. Arul Kumar ${ }^{2}$

C. Matthews ${ }^{2}$

M. C. Messner ${ }^{3}$

A. Chakraborty

August 2020

${ }^{1}$ Computational Mechanics and Materials, Idaho National Laboratory

${ }^{2}$ Materials Science and Technology Division, Los Alamos National Laboratory

${ }^{3}$ Argonne National Laboratory

Prepared for the

U.S. Department of Energy

Office of Nuclear Energy

Under DOE Idaho Operations Office

Contract DE-AC07-05ID14517 


\section{Contents}

$\begin{array}{lr}\text { Executive Summary } & 6\end{array}$

$\begin{array}{llr}1 & \text { INTRODUCTION } & 7\end{array}$

2 DEVELOPMENT OF UNCERTAIN PARAMETERS FOR PHENOMENOLOGICAL MODELS OF HIGH TEMPERATURE CREEP RESPONSE $\quad \mathbf{9}$

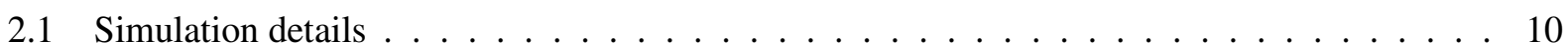

2.1 .1 Inelastic Material Model . . . . . . . . . . . . . . . . . . . . 10

2.1 .2 Bayesian Analysis . . . . . . . . . . . . . . . . . . . . 11

2.2 Results . . . . . . . . . . . . . . . . . . . . . . . 12

2.2.1 Monotonic Model . . . . . . . . . . . . . . . . . . . . . . . 12

2.2.2 Bayesian MCMC with Real Data . . . . . . . . . . . . . . . . . . . . . . . . . . . . . 13

2.2 .3 Cyclic Model . . . . . . . . . . . . . . . . . . . . 13

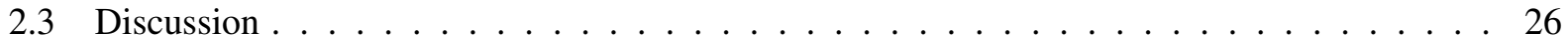

2.3 .1 Uniaxial Model . . . . . . . . . . . . . . . . . . . 26

2.3 .2 Cyclic Model . . . . . . . . . . . . . . . . . . . . 26

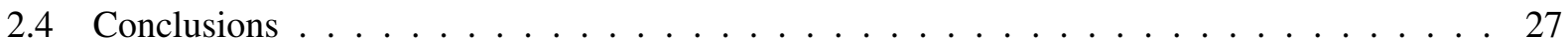

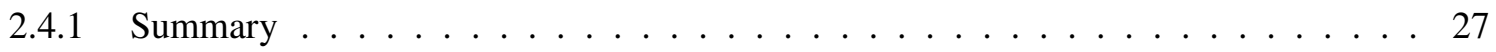

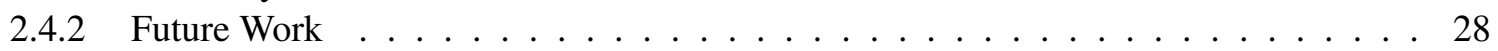

3 MICROSTRUCTURE-BASED REDUCED ORDER MODEL FOR ENGINEERING-SCALE CONSTITUTIVE RESPONSE $\quad 32$

3.1 Crystal Plasticity Constitutive Model . . . . . . . . . . . . . . . . . . . . . 32

3.1.1 Waiting Time for Thermally Activated Glide . . . . . . . . . . . . . . . . 34

3.1 .2 Waiting Time For Climb Assisted Glide . . . . . . . . . . . . . . . . . . . 35

3.1 .3 Coble Creep . . . . . . . . . . . . . . . . . . . 35

3.1 .4 Dislocation Density Evolution . . . . . . . . . . . . . . . . 36

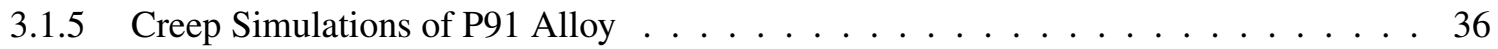

3.2 Database Generation . . . . . . . . . . . . . . . . . . . . . . . 40

3.3 Surrogate Model Formulation . . . . . . . . . . . . . . . . . . . 41

3.4 Surrogate Model Validation . . . . . . . . . . . . . . . . . . . . . . . . . . . . . . . . . 45

3.4 .1 Creep Test Validation . . . . . . . . . . . . . . . . . . . . . 45

3.4 .2 Complex Loading Conditions . . . . . . . . . . . . . . . 45

4 Uncertainty Quantification of Engineering-Scale Component Life Predictions 50

4.1 NEML Material Model Results . . . . . . . . . . . . . . . . . . . . . . 51

$4.1 .1 \quad$ NEML Simplified Pipe Model Results . . . . . . . . . . . . . . . . . . . 53

4.1.2 NEML Sphere Nozzle Model Results . . . . . . . . . . . . . . . . . . . . 57

4.2 LAROMANCE Material Model Results . . . . . . . . . . . . . . . . . . . . . 60

4.2.1 LAROMANCE Simplified Pipe Model Results . . . . . . . . . . . . . . . . . . 61

4.2.2 LAROMANCE Sphere Nozzle Model Results . . . . . . . . . . . . . . . . . . . . 66

4.3 Discussion . . . . . . . . . . . . . . . . . . . . 72 
5 Summary and Future Work

Acknowledgments 


\section{Executive Summary}

This report summarizes a joint effort between Argonne National Laboratory, Idaho National Laboratory, and Los Alamos National Laboratory to develop and deploy constitutive models targeted at predicting the life of Grade 91 alloy components subjected to high temperature environments typical of those that structural components in advanced nuclear reactors would experience.

Two distinct, but complementary constitutive modeling approaches have been taken here. The first employs a phenomenological viscoplastic model for which parameters have been calibrated based on experimental data for a wide range of Grade 91 alloy that has undergone a variety of processing. A Bayesian approach was used to derive distributions of uncertain parameters for this model based on this data set.

The second approach is a reduced order model suitable for engineering-scale analysis that is based on the results of a large set of mesoscale simulations. Mesoscale models allow for the microstructure and composition of a particular alloy to be directly taken into account in the computation of the viscoplastic response, but are computationally expensive, which makes it impractical to directly call those models for the material constitutive response in an engineering-scale simulation. The reduced-order representation of the response of the underlying model used here allows for an engineering-scale model to take into account the characteristics of the underlying microstructure while only incurring a reasonable computational expense.

Both of these approaches have different strengths and are applicable for different parts of the design/analysis process. The phenomenological models can be readily parameterized based on a set of experimental data for a given class of materials and used for scoping calculations. Once a specific material is chosen and adequately characterized, the reduced order models can accurately predict the response of that specific alloy, and because the models are based on predictive models of the underlying microstructure, they can be used to more confidently predict the response under conditions in regions where there is limited experimental data.

Both of these models have been integrated in the Grizzly code, which is used here to perform proof-ofconcept uncertainty quantification analyses of a simple component under prototypical conditions. The builtin stochastic analysis capabilities in the MOOSE framework that Grizzly is built on are used here to run large sets of simulations for this uncertainty quantification analysis. As would be expected, because the reduced order models are developed for a much more tightly defined alloy, they predict tighter distributions of the time to failure than the phenomenological models, which are calibrated to a broader set of data. Also important is that these simulations demonstrate that a reduced order modeling approach can be successfully deployed to propagate uncertainties from the material scale to practical engineering-scale component simulations. 


\section{INTRODUCTION}

Multiple concepts are in development for advanced reactors that offer improved economics and safety relative to the light water reactors (LWRs) that currently provide a major portion of the clean energy supplied to the electrical grid in the United States. Most of these designs operate at higher temperatures than LWRs, which places unique demands on their structural components.

There is an ongoing effort by the US Department of Energy Office of Nuclear Energy's Modeling and Simulation Program to develop simulation tools to predict the behavior of these advanced reactors, including fuel performance, reactor physics, and thermal hydraulics. A viable reactor design must not only have adequate safety and performance in these areas, but the structural components that contain the reactor must also be able to adequately withstand the harsh environmental conditions of the reactor.

Efforts have been underway for several years to develop capabilities to simulate the aging of structural components in the Grizzly code, which is developed at Idaho National Laboratory (INL). This development originally focused on LWRs, but more recently has shifted to also address material issues in advanced reactors. Grizzly is based on INL's MOOSE multiphysics simulation framework and uses the finite element method in multiple dimensions to simulate both the progression of aging mechanisms and the effects of that aging on nuclear power plant structures.

One of the major issues of concern for advanced reactor structural components is creep, which is a much larger concern at the elevated temperatures for those applications than it is for LWR applications. To address high-temperature creep in advanced reactor metals, two fundamentally different types of material constitutive models have been integrated into Grizzly.

The first approach uses traditional phenomenological models, the parameters of which are calibrated to match experimental observations of the response of a material of interest in a given regime. These models are provided through the Nuclear Engineering material Model Library (NEML), which is an open-source library of common classical material models that can be combined in a variety of ways. This library, developed at Argonne National Laboratory (ANL), can optionally be linked with Grizzly. These models have the advantage that their behavior is generally well-understood because they have been in use by the engineering community for a long time, and they can perform well if they are used in the regime for which they have been calibrated. They are also relatively simple, so they offer good robustness and computational performance. However, because the parameters that define their behavior are based on the observed response of the material, rather than the underlying microstructure, they cannot readily be used to account for changes in the microstructure, such as those that occur due to irradiation, and their use outside the regime for which they were calibrated is also limited.

The second class of material models explored here are reduced order models (ROMs) that are derived from mining a synthetic database of predicted mechanical response derived from the use of a crystal plasticity based mechanistic constitutive model. The steps for ROM development include:

- Meso-scale polycrystal model is calibrated using the available limited experimental data

- Synthetic database of material response (e.g., creep behavior and the evolution of microstructure) is developed for a wide range of design interest.

- $\mathrm{ROM}$ is developed from the database using orthogonal polynomial regressions. 
The visco-plastic self-consistent (VPSC) polycrystal plasticity model can be used to compute the response of a representative volume of material under mechanical loading and account for the effects of microstructural features. Directly evaluating a mesoscale model at every material point in an engineering-scale model is possible, but cost-prohibitive. To provide the benefits of a mesoscale-based model that is aware of microstructure, a mesoscale model is evaluated under a wide range of conditions of interest, and data analytics are used to develop an ROM that represents the material response using functions that are readily evaluable within an engineering constitutive model. A set of such models, known as LAROMANCE, for a growing number of alloy types have been developed by Los Alamos National Laboratory (LANL).

The present report describes these two constitutive modeling approaches, and then demonstrates their application in an engineering-scale analysis to Grade 91 alloy, one of the six alloys currently permitted for high temperature nuclear applications by the ASME Boiler and Pressure Vessel Code. Ultimately, an engineering analysis of a component in a high temperature environment will be concerned with predicting the time until excessive creep or creep rupture occurs. Quantifying the uncertainty in this component lifetime requires a significant number of engineering-scale calculations, which require robust, usable constitutive models.

For the phenomenological NEML model, extensive work was performed to characterize the uncertainty of the parameters for that model through the use of Bayesian statistics. For the microstructure-based LAROMANCE model, the uncertain parameters are based on the properties of the microstructure, rather than on experimental observation of the material response. The components chosen for this study are relatively simple, as are the failure criteria. This study, which is a joint effort of three national laboratories (INL, ANL, and LANL), is intended to be a proof of concept to demonstrate how uncertain parameters can be computed and used for a phenomenological model, and how uncertainty in the underlying microstructure parameters can be propagated to the engineering response in the case of the ROM.

This report begins by providing details of the phenomenological model for Grade 91 and the development of the uncertain parameters for that model in Chapter 2. Next, it provides an overview of the ROM in Chapter 3. Application of these models to engineering-scale uncertainty quantification analysis is described in Chapter 4, followed by a summary and recommendations for future work in Chapter 5. 


\section{DEVELOPMENT OF UNCERTAIN PARAMETERS FOR PHENOMENOLOGICAL MODELS OF HIGH TEMPERATURE CREEP RESPONSE}

A better understanding of uncertainties in structural degradation of components operating at high temperatures could lead to better engineering design. Except for a few relatively recent studies (c.f. [1-3]) most existing analysis involving a probabilistic approach for high temperature materials focus on crack growth under the creep boundary conditions $[4,5]$. Moreover, probabilistic methods are rarely used in the design of high temperature components. Several factors limit the application of probabilistic methods to high temperature components:

1. Limited experimental test data

2. Poorly-quantified loading conditions

3. Analysis methods that isolate single components from plant systems

However, applying statistical analysis to inelastic material behavior is likely the most significant obstacle.

In this chapter we describe an initial method for quantifying the uncertainties in high temperature inelastic material response by formulating an empirical statistical model for both uniaxial and cyclic loading conditions.

Parameter sets for deterministic inelastic models typically minimize the difference between model prediction and experimental data, known as the inverse analysis [6]. There is extensive previous work focusing on estimating inelastic constitutive model parameters deterministically, with studies ranging from simple isotropic one-dimensional models to complex anisotropic microstructure-informed crystal plasticity models with different optimization algorithms [7-18]. An important drawback of such deterministic approaches is that they neglect the observed scatter in experimental data arising due to measurement noise or material heat-to-heat property variations, which can only be addressed through statistical modeling [19].

This work considers a Bayesian inverse analysis of conventional, empirical material models. Bayesian analysis results in the probability distributions of the material parameters of interest, called the posterior distributions, starting from an initial guess, termed the prior distributions. This methodology incorporates the existing knowledge of the system through these prior distributions and updates the priors using the likelihood function to finally generate the posterior distributions following the Bayes' rule. Most often, the Bayesian analysis uses the Markov Chain Monte Carlo (MCMC) algorithm for sampling points in the parameter space. A few previous studies exist in literature incorporating Bayesian MCMC methodology to identify parameters for inelastic models, including [20-27]. However, none of these studies considers a complete high temperature constitutive model suitable for representing combined creep and plastic deformation.

This report applies the Bayesian MCMC algorithm to develop statistical models for the steady state and cyclic behavior of Grade 91 steel at elevated temperatures. Previous works have been devoted to developing and calibrating deterministic constitutive models to capture this behavior [15-17]. However, there is a wide scatter in the experimental data arising both from material variability as well as differences in the experimental conditions. A statistical model could capture the observed scatter in the experimental data through a statistical distribution of the underlying model parameters. 
In this chapter we first apply the Bayesian MCMC on a simplified uniaxial inelastic model using tensile and creep reference data. Subsequently, we incorporate strain controlled cyclic tests to generate a unified statistical model including kinematic hardening parameters, but excluding cyclic damage.

\subsection{Simulation details}

This section describes the deterministic inelastic material models used to perform the statistical modeling, as well as gives a brief overview of the Bayesian MCMC methodology.

\subsubsection{Inelastic Material Model}

The models considered here are viscoplastic:

$$
\dot{\epsilon}=\dot{\epsilon}_{e}+\dot{\epsilon}_{v p}
$$

with the elastic strain rate following Hooke's law

$$
\dot{\epsilon}_{e}=E \dot{\sigma}
$$

where $E$ is the Young's modulus. This section considers two variants of a basic viscoplastic model: one suitable only for monotonic loading but including the effect of creep damage on the evolution of the creep rate and one suitable for high temperature cyclic plasticity, but excluding the development of creep-fatigue damage.

\section{Monotonic Model with Damage}

We first test the Bayesian methodology on a simplified uniaxial elasto-viscoplastic damage model. For this model the inelastic strain rate follows the Perzyna flow rule [28] with damage,

$$
\dot{\epsilon}_{v p}=\left\langle\frac{\frac{\sigma}{1-\omega}-\sigma_{0}-R}{\eta}\right\rangle^{n},
$$

where $\omega$ is the damage variable, and $n, \eta$ and $\sigma_{0}$ are the viscoplastic parameters. The hardening internal variable $R$ follows a standard isotropic Voce hardening law, [29],

$$
R=Q\left[1-\exp \left(-b\left|\epsilon_{v p}(t)\right|\right)\right]
$$

where $b$ and $Q$ are the hardening parameters. The damage rate is the classical model by Leckie and Hayhurst [30],

$$
\dot{\omega}=\left(\frac{\sigma}{A}\right)^{\zeta}(1-\omega)^{-\phi}
$$

where $A, \phi$ and $\zeta$ represents the adjustable damage parameters.

\section{Inelastic Model for Cyclic Loading}

We also test the applicability of the Bayesian approach when including more complex cyclic tests (along with tensile and creep experiments) involving kinematic hardening parameters. In this preliminary study, we 
exclude damage in this variant to simplify the equations and reduce the number of model parameters. The inelastic strain rate (without damage) thus follows the Chaboche flow rule with backstress, [29],

$$
\epsilon_{v p}^{\cdot}=\left\langle\frac{|\sigma-\kappa|-R}{\eta}\right\rangle^{n} \operatorname{sign}(\sigma-\kappa),
$$

where the backstress, $\kappa$, is the sum of two Chaboche backstresses:

$$
\begin{aligned}
\kappa & =\sum_{i}^{2} \kappa_{i}, \\
\dot{\kappa}_{i} & =C_{i} \epsilon_{v p}^{\cdot}-\gamma_{i} \kappa_{i}\left|\epsilon_{v p}^{\cdot}\right|
\end{aligned}
$$

We neglect the recovery terms in the evolution equation of the backstresses while the hardening internal variable, $R$, follows the same Voce isotropic hardening rule as discussed earlier in Section 2.1.1. The cyclic model increases the dimensionality of the problem with four additional backstress terms $\left(C_{1}, C_{2}, \gamma_{1}, \gamma_{2}\right)$, as well as the computational cost when solving the cyclic boundary condition.

\subsubsection{Bayesian Analysis}

In this work we capture the uncertainties in the material response by generating statistical parameter models using Bayesian MCMC analysis. The algorithm [31] updates the probability of the model parameters, $\boldsymbol{\alpha}$, given by the posterior distribution $\boldsymbol{\pi}(\boldsymbol{\alpha} \mid D)$ using Bayes' theorem applied to the prior distribution, $\boldsymbol{\pi}_{\mathbf{0}}(\boldsymbol{\alpha})$, describing whatever prior knowledge is available for the system and the data $D$ :

$$
\pi(\alpha \mid D)=\frac{\pi(D \mid \alpha) \pi_{0}(\alpha)}{\pi(D)}
$$

The likelihood, $\pi(D \mid \boldsymbol{\alpha})$ is the probability of observing the data, $D$, given the parameter realizations, $\boldsymbol{\alpha}$. Most method treat $\pi(D)$, which represents the probability of the data, as a normalization factor, giving the simplified update

$$
\pi(\alpha \mid D) \propto \pi(D \mid \alpha) \pi_{0}(\alpha)
$$

The analysis assumes $\pi(D \mid \boldsymbol{\alpha})$ similar to [26], and its logarithm, $\mathcal{L}$, represents the likelihood function determining the acceptance or rejection of the sampled parameters,

$$
\begin{aligned}
\pi(D \mid \boldsymbol{\alpha}) & =\frac{1}{\left(2 \pi \xi^{2}\right)^{\frac{n}{2}}} \exp \sum_{i=1}^{n}\left(\frac{-\left(y_{i}-g_{i}(\boldsymbol{\alpha})\right)^{2}}{2 \xi^{2}}\right), \text { or }, \\
\mathcal{L} & =\log (\pi(D \mid \boldsymbol{\alpha})) \equiv \sum_{i=1}^{n}\left(\frac{-\left(y_{i}-g_{i}(\boldsymbol{\alpha})\right)^{2}}{2 \xi^{2}}\right)
\end{aligned}
$$

where $\xi$ is the data scatter, treated as a hyperparameter. $i$ iterates over the different sets of experimental data. $y_{i}$ represents (synthetic or real) experimental results at the different test conditions (for tension, creep, and cyclic tests), and $g_{i}(\boldsymbol{\alpha})$ represents the corresponding simulated results with the sampled parameter values $\boldsymbol{\alpha}$. This estimation procedure here uses the open-source Python package PyMC3 [32] with the MetropolisHastings algorithm [33] and the Monte-Carlo sampling [34]. The analysis runs several Markov chains to test convergence. 


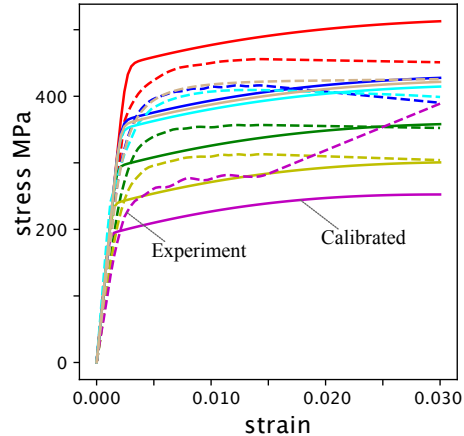

(a) $550 \mathrm{C}$

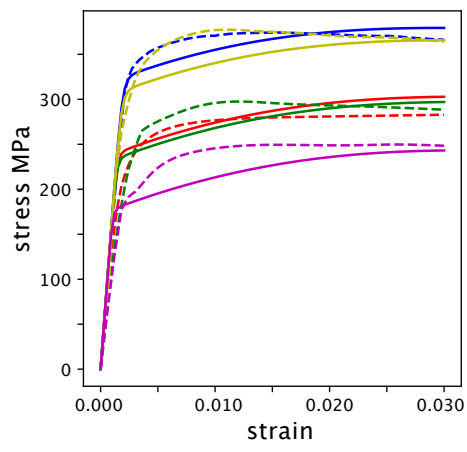

(c) $600 \mathrm{C}$

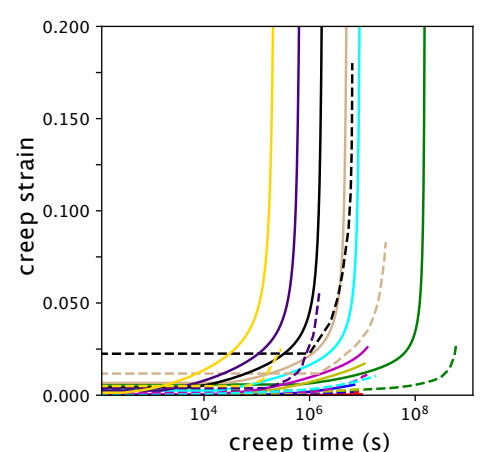

(b) $550 \mathrm{C}$

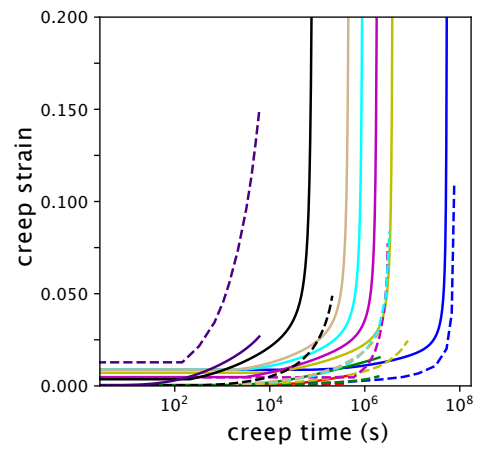

(d) $600 \mathrm{C}$

Figure 2.1: Calibrated model for Grade 91 at $550{ }^{\circ} \mathrm{C}(\mathrm{a}, \mathrm{b})$ and at $600{ }^{\circ} \mathrm{C}(\mathrm{c}, \mathrm{d})$. In the figures solid lines are model results and dashed lines are experiments. Different colors represent different experimental conditions, i.e., strain rates for tensile tests and stresses for creep tests.

\subsection{Results}

This section tests the performance of the Bayesian analysis against real experimental data of Grade 91 at 550 and $600{ }^{\circ} \mathrm{C}$.

\subsubsection{Monotonic Model}

The calibration database for the model includes results from uniaxial tension and creep experiments at multiple strain rates and creep stresses drawn from the literature [35-41]. For those cases with more than one set of experimental data at the same test conditions, we consider a mean (numerical average) response for both model calibration and the Bayesian MCMC analysis. Solid lines in Figure 2.1 are model predictions; dashed lines are experimental data. The initial guess is a deterministic genetic algorithm (GA) fit to the experimental data, minimizing the sum of the squared error between model predictions and experimental results. Each experiment receives a weight factor so that the tension and creep data weigh equally in the initial objective function value. The GA calibrates only the five inelastic model parameters while (as previously mentioned) the linear Larson-Miller correlations provide the damage parameter values. We calibrate the model separately at 550 and $600{ }^{\circ} \mathrm{C}$ because the model parameters can depend on temperature. Figure 2.1 shows the calibrated mean-property model results, and Table 2.1 shows the calibrated model parameters $(\mu$ in columns 2 and 4) at the two temperatures. The Bayesian MCMC analysis assumes that the parameters follow a truncated normal distribution (an informed prior) with prior means corresponding to the calibrated values and assumed standard deviations listed in Table 2.1. We select the parameter bounds (also listed in Table 2.1 such that they center the calibrated mean values. Moreover, for the sake of completeness we also 
Table 2.1: Prior (normal) distributions of the model parameters with their respective means, $\mu$, standard deviations, and bounds for the Bayesian MCMC analysis.

\begin{tabular}{cccccc}
\hline \multirow{2}{*}{ parameter } & \multicolumn{2}{c}{$550^{\circ} \mathrm{C}$} & & \multicolumn{2}{c}{$600^{\circ} \mathrm{C}$} \\
\cline { 2 - 3 } \cline { 5 - 6 } & $(\mu$, std. dev $)$ & Bounds & & $(\mu$, std. dev $)$ & Bounds \\
\hline$n$ & $(10.81,0.5)$ & {$[9,13]$} & & $(8.274,0.6)$ & {$[7,10]$} \\
$\eta$ & $(844.6,27.0)$ & {$[700,950]$} & & $(747.4,57.0)$ & {$[600,900]$} \\
$\sigma_{0}$ & $(3.667,0.8)$ & {$[2,10]$} & & $(3.550,0.7)$ & {$[2,5]$} \\
$Q$ & $(106.0,9.0)$ & {$[80,130]$} & & $(112.0,9.0)$ & {$[80,130]$} \\
$b$ & $(45.43,9.0)$ & {$[40,70]$} & & $(44.33,9.0)$ & {$[20,70]$} \\
$A$ & $(925.0,20.0)$ & {$[800,1000]$} & & $(650.6,20.0)$ & {$[600,700]$} \\
$\zeta$ & $(11.36,1.0)$ & {$[10,15]$} & & $(10.71,1.0)$ & {$[9,14]$} \\
$\phi$ & $(2.04,0.5)$ & {$[1,3]$} & & $(2.04,0.5)$ & {$[1,3]$} \\
\hline
\end{tabular}

vary the damage parameters during the Bayesian analysis, increasing the dimensionality of the problem.

\subsubsection{Bayesian MCMC with Real Data}

Using the assumed prior distributions (mean, standard deviations, and parameter bounds) as listed in Table 2.1, we run the Bayesian analysis for all of the eight (five inelastic and three damage) model parameters. We select $\xi=200.0$ and $\xi=0.01$ for the real experimental uniaxial and creep conditions for both the temperatures, based on experiments with synthetic data. Figure 2.2 shows the posterior distributions obtained from the Bayesian MCMC analysis for Grade 91 at the two temperatures. The final posterior distributions are all approximately normal. Table 2.2 summarizes the results obtained from the Bayesian MCMC study with the

Table 2.2: Prior and posterior means of the model parameters.

\begin{tabular}{cccccc}
\hline parameter & \multicolumn{2}{c}{$550^{\circ} \mathrm{C}$} & & \multicolumn{2}{c}{$600^{\circ} \mathrm{C}$} \\
\cline { 2 - 3 } \cline { 5 - 6 } \cline { 5 - 6 } & prior mean & posterior mean & & prior mean & posterior mean \\
\hline$n$ & 10.81 & 11.0 & & 8.274 & 8.5 \\
$\eta$ & 844.6 & 832.0 & & 747.4 & 751.0 \\
$\sigma_{0}$ & 3.66 & 3.88 & & 3.550 & 3.5 \\
$Q$ & 106.0 & 107.0 & & 112.0 & 113.0 \\
$b$ & 45.43 & 48.0 & & 44.33 & 44.0 \\
$A$ & 924.9 & 927.0 & & 650.6 & 656.0 \\
$\zeta$ & 11.36 & 12.0 & & 10.71 & 12.0 \\
$\phi$ & 2.04 & 2.0 & & 2.04 & 2.0 \\
\hline
\end{tabular}

uniaxial material model. Clearly, the prior and the posterior means are close to each other, indicating the benefit of initial model calibration with GA optimization followed by Bayesian analysis. Figure 2.3 shows simulations of 30 random samples from the posterior distributions for each creep and tensile curve, overlaid on the experimental data. Figure 2.4 compares the distributions of the simulated $0.2 \%$ offset stresses (yield strength) and the simulated failure times, again compared to the corresponding experimental values.

\subsubsection{Cyclic Model}

This section includes strain controlled cyclic tests to obtain the statistical model for a unified viscoplastic model for cyclic plasticity. First we test the efficacy of the Bayesian analysis in recovering accurate material behavior using synthetically generated tensile, creep, and strain controlled cyclic data at multiple conditions. Subsequently, we implement the Bayesian MCMC for real experimental data of Grade 91 at $550{ }^{\circ} \mathrm{C}$. Table 2.3 

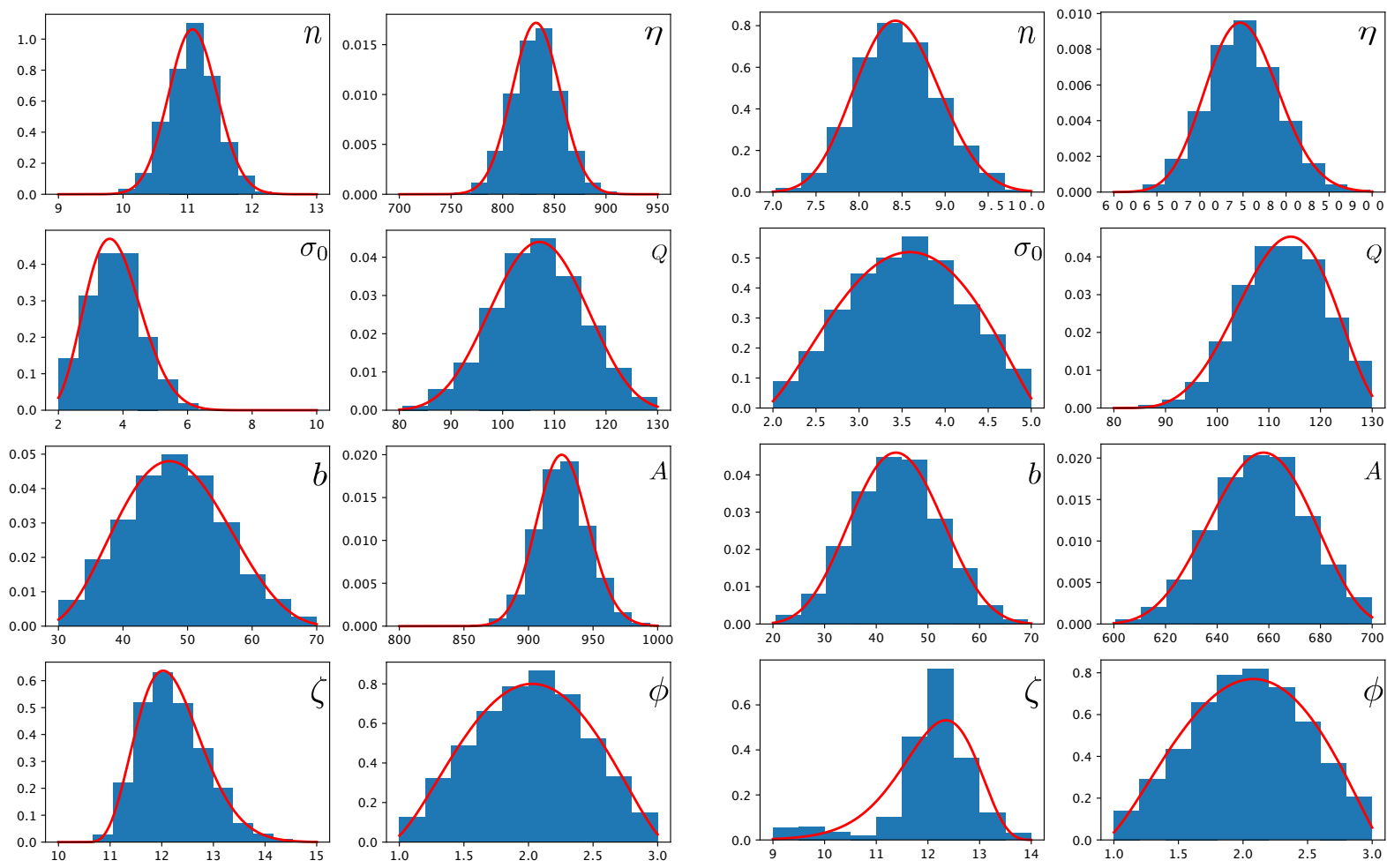

(a) $550 \mathrm{C}$

(b) $600 \mathrm{C}$

Figure 2.2: Posterior distributions of each of the 8 model parameters, $n, \eta, \sigma_{0}, Q, b, A, \zeta, \phi$, at $550^{\circ} \mathrm{C}$, (a), and $600^{\circ} \mathrm{C},(\mathrm{b})$. The histograms are shown in blue while an analytical fit with a beta distribution for the given data is represented by the red curves. The abscissae span the bounds of each parameter while the ordinates are normalized such that the integral of their probability density is 1 , to include the histograms and the probability density function (red curve) on the same plot. All parameters appear to have a near normal distribution. 

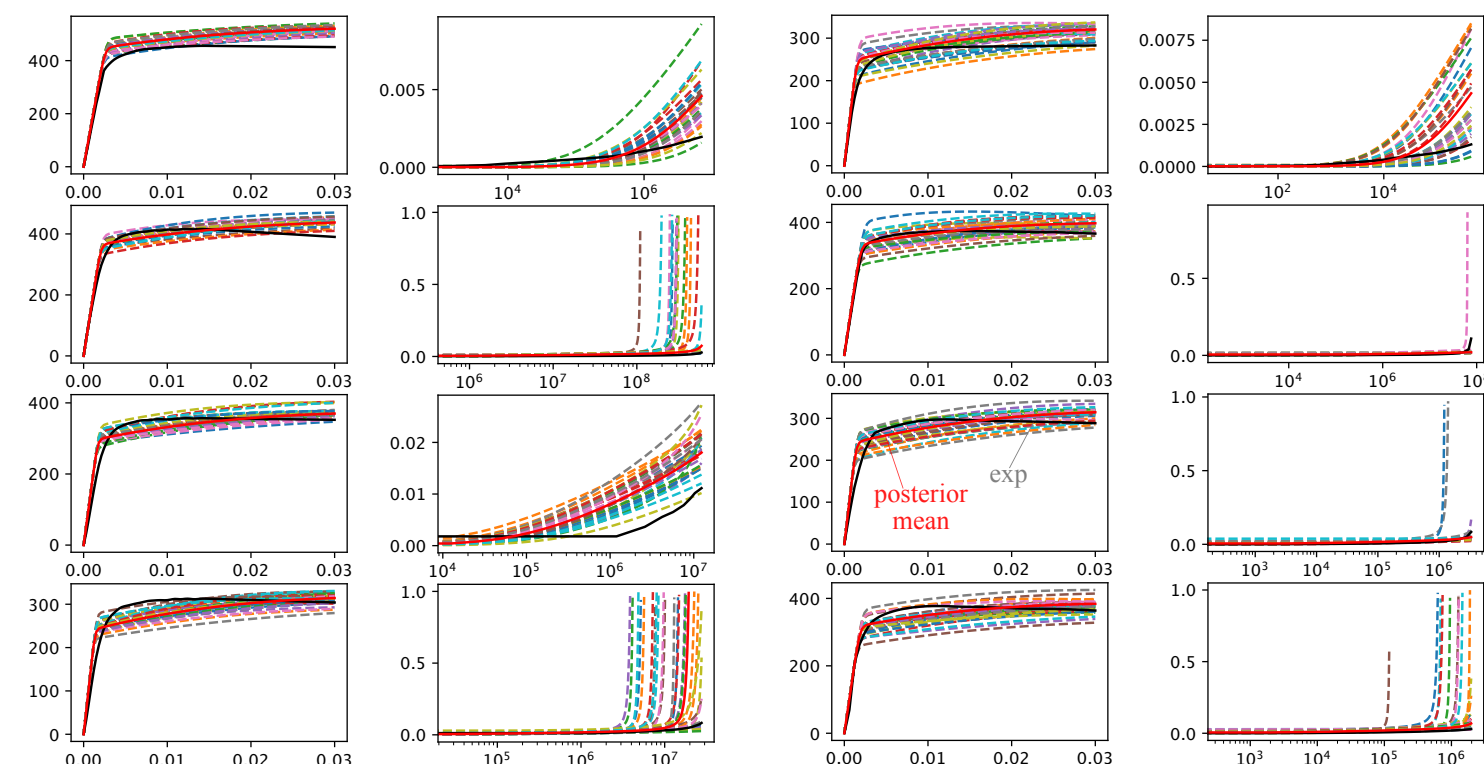

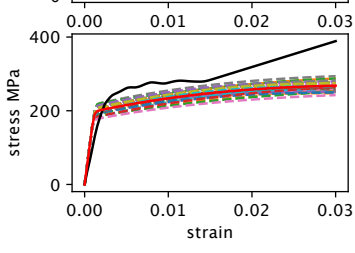

(a)

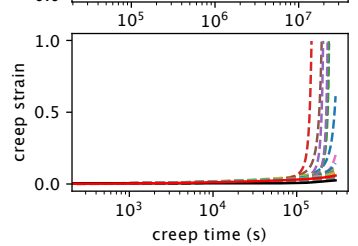

(b)
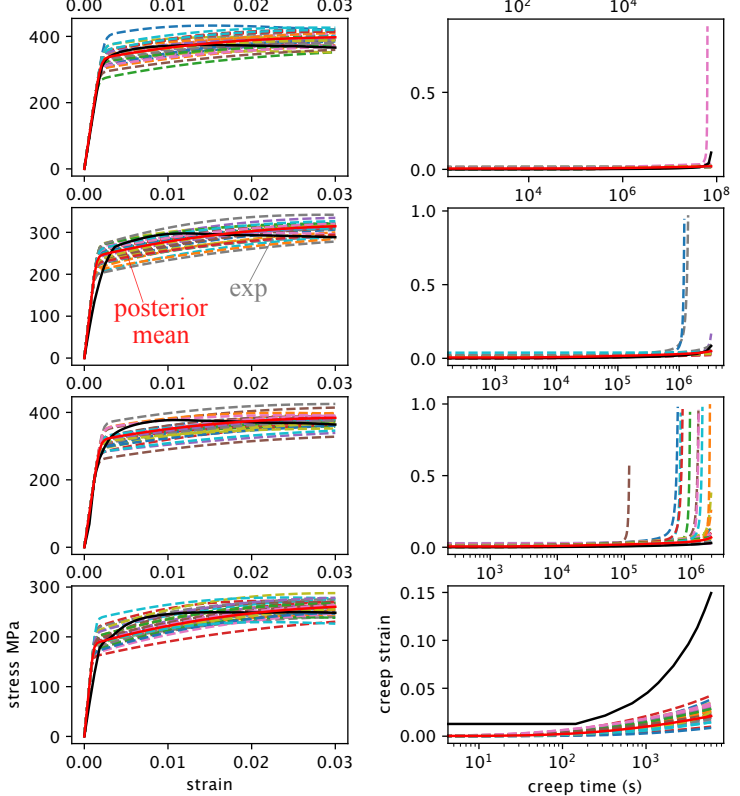

(c)

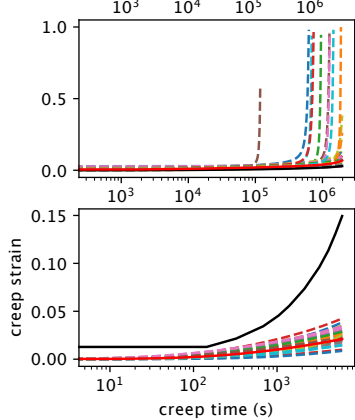

(d)

Figure 2.3: Spread in the output of 30 randomly sampled models at $550{ }^{\circ} \mathrm{C}$, (a) tensile, (b) creep, and at $600^{\circ} \mathrm{C}$, (c) tensile, (d) creep. Solid black curves represent the experimental data while the solid red curves represent the simulated mean result. This figure shows a subset of the total dataset for brevity; however, a similar trend is observed for all the cases.

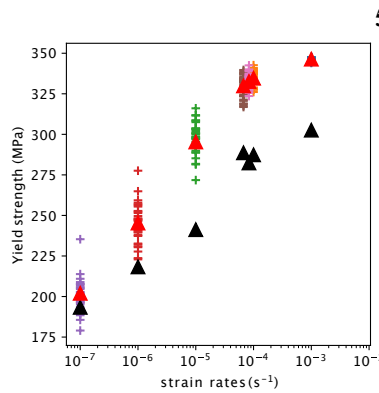

(a)

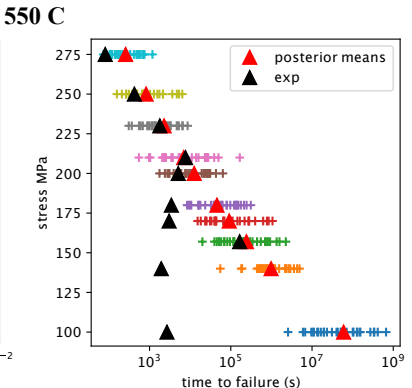

(b)

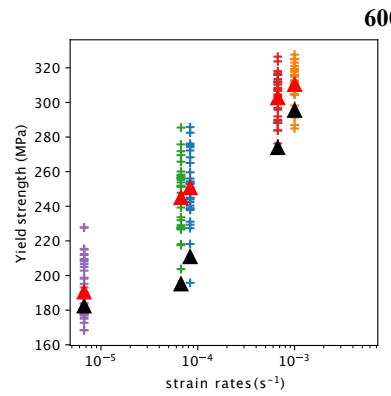

(c)

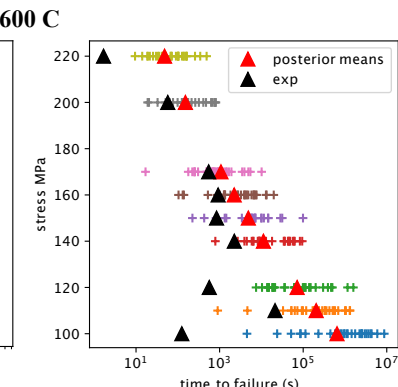

(d)

Figure 2.4: Scatter in yield strengths ((a), (c)) and failure times ((b), (d)) from the simulated tensile and creep tests by sampling parameter sets from the posterior distribution (dashed curves in Figure 2.3 at $550{ }^{\circ} \mathrm{C}((\mathrm{a}),(\mathrm{b}))$ and at $600{ }^{\circ} \mathrm{C}((\mathrm{c}),(\mathrm{d}))$. The yield strength and failure time results using the posterior mean parameter values are shown by red triangular markers while the black triangles corresponding to their equivalent experimental values. 
Table 2.3: Bounds for the inelastic parameters of the cyclic model.

\begin{tabular}{cc}
\hline Parameter & Bounds \\
\hline$n$ & {$[9,13]$} \\
$\eta$ & {$[700,950]$} \\
$Q$ & {$[90,110]$} \\
$b$ & {$[3,6]$} \\
$C_{1}$ & {$\left[120.0 \times 10^{3}, 160.0 \times 10^{3}\right]$} \\
$C_{2}$ & {$\left[100.0 \times 10^{3}, 110.0 \times 10^{3}\right]$} \\
$\gamma_{1}$ & {$\left[90.0 \times 10^{3}, 110.0 \times 10^{3}\right]$} \\
$\gamma_{2}$ & {$\left[5.0 \times 10^{2}, 11.0 \times 10^{2}\right]$} \\
\hline
\end{tabular}

lists the inelastic parameter bounds of the (cyclic) inelastic material model to generate the synthetic data. Figure 2.5 shows synthetic experimental data for the tensile and creep tests with seven different test conditions generated with inelastic parameter values sampled from an uncorrelated normal distribution, with mean 0.5 and standard deviation of 0.1 within bounds listed in Table 2.3 when scaled to [0,1].

\section{Effect of Prior Means}

Figure 2.6 compares the posterior distributions obtained from two different prior distributions with mean and standard deviation of 0.3 and 0.2 , (a), and 0.7 and 0.2 , (b), respectively. The bounds for the Bayesian MCMC are the same as those used to generate the synthetic reference data (listed in Table 2.3). Clearly, the posterior means for the parameters $n$ and $\eta$ converge to their target values while, for other parameters, the posteriors do not change significantly from their priors. Figure 2.7 shows the predicted responses with the posterior mean parameter values as well as the corresponding synthetic reference values. Even though the posterior distributions of the hardening parameters (isotropic and kinematic) are away from their true values, the material response is accurate and close to the reference. The lack of change for the priors of these hardening parameters can then be attributed to relatively narrow bounds for the hardening parameters, especially the kinematic hardening parameters.

\section{Effect of Prior Bounds}

Subsequently, we increase the bounds of the kinematic hardening parameters, $C_{1}, C_{2}, \gamma_{1}$, and $\gamma_{2}$ from those listed in Table 2.3 to $\left[1 \times 10^{2}, 2 \times 10^{5}\right]$ for all. Figure 2.8 demonstrates the effectiveness of the Bayesian MCMC methodology to clearly recover the true solutions in the parameter space because there is a significant difference between the prior means $(0.3)$ and posterior means of the kinematic hardening parameters.

\section{Real Experimental Data}

Similar to the uniaxial material model, we perform the Bayesian MCMC analysis with real tensile, creep, and strain controlled cyclic data of Grade 91 at $550^{\circ} \mathrm{C}$.

Model calibration The calibration database for the cyclic model includes experiments at multiple tensile strain rates and creep stresses, and cyclic strain rates and strain amplitudes, [35-41]. We use the GA optimization module in SciPy to perform an initial calibration of the model with real experimental data. Table 2.4 lists the parameter bounds for the GA optimization. Figure 2.9 shows the calibrated model with the real ex- 


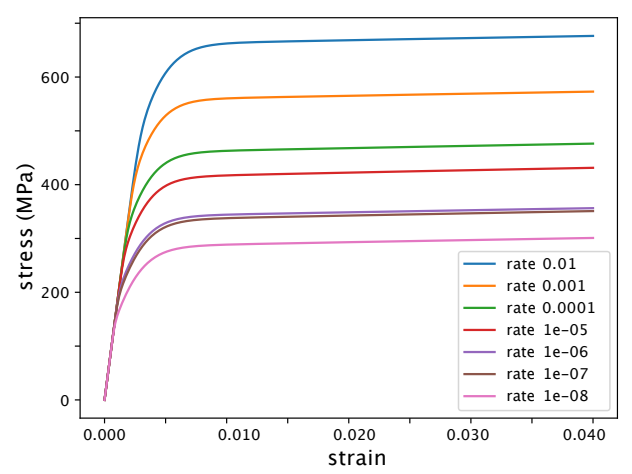

(a)

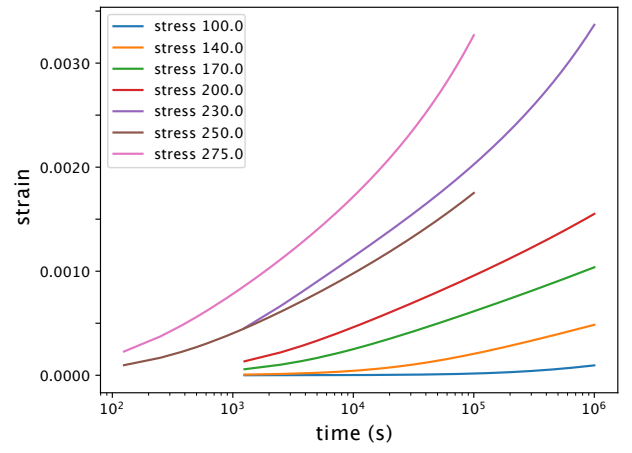

(b)

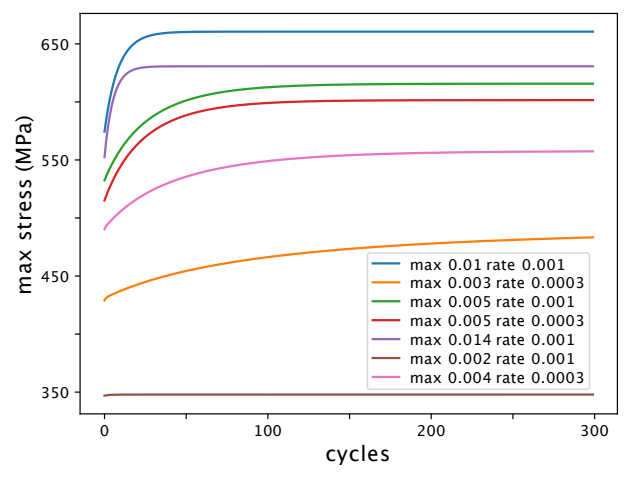

(c)

Figure 2.5: Synthetic experimental data for 7 different tensile, (a), creep, (b), and (c) strain controlled cyclic tests. Different colors correspond to different test conditions, i.e., strain rates (labeled as rate in the figures) for tensile tests, stresses for creep tests, and a combination of strain rates (rates) and strain amplitudes (labeled max) for the cyclic tests. 

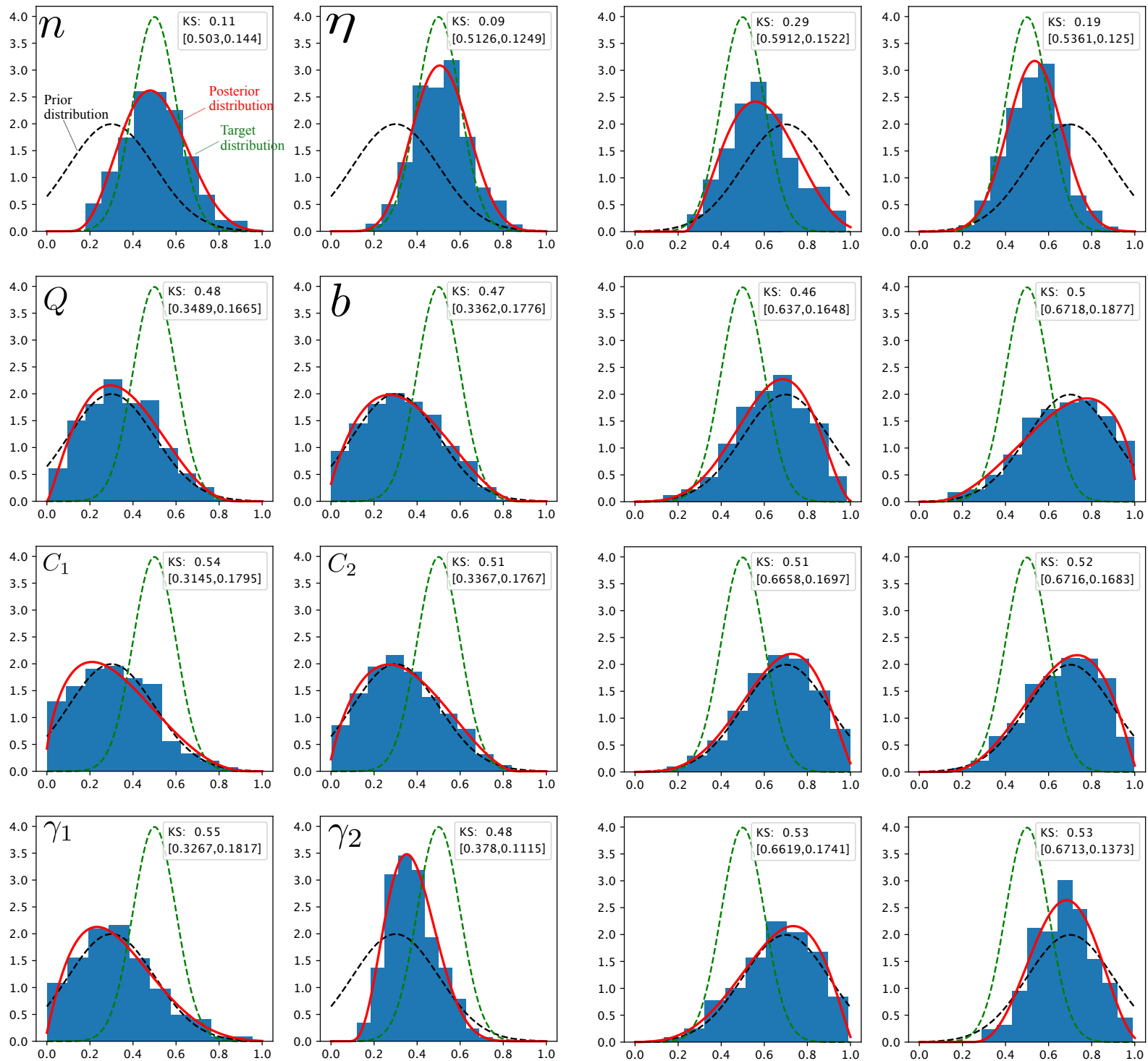

(a)
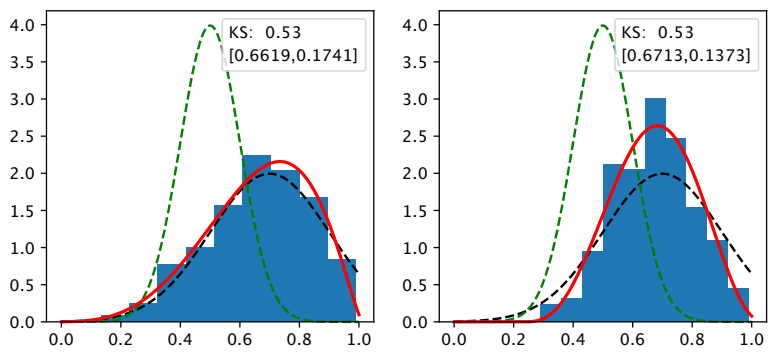

(b)

Figure 2.6: Posterior distributions from truncated normal priors with mean 0.3, (a), and 0.7, (b), and standard deviations of 0.2 (for both), for the inelastic parameters of the cyclic model. The respective prior and target distributions are shown in black and red curves respectively, as well as the KolmogorovSmirnov (KS) values and the posterior means and standard deviations in the top corners. 

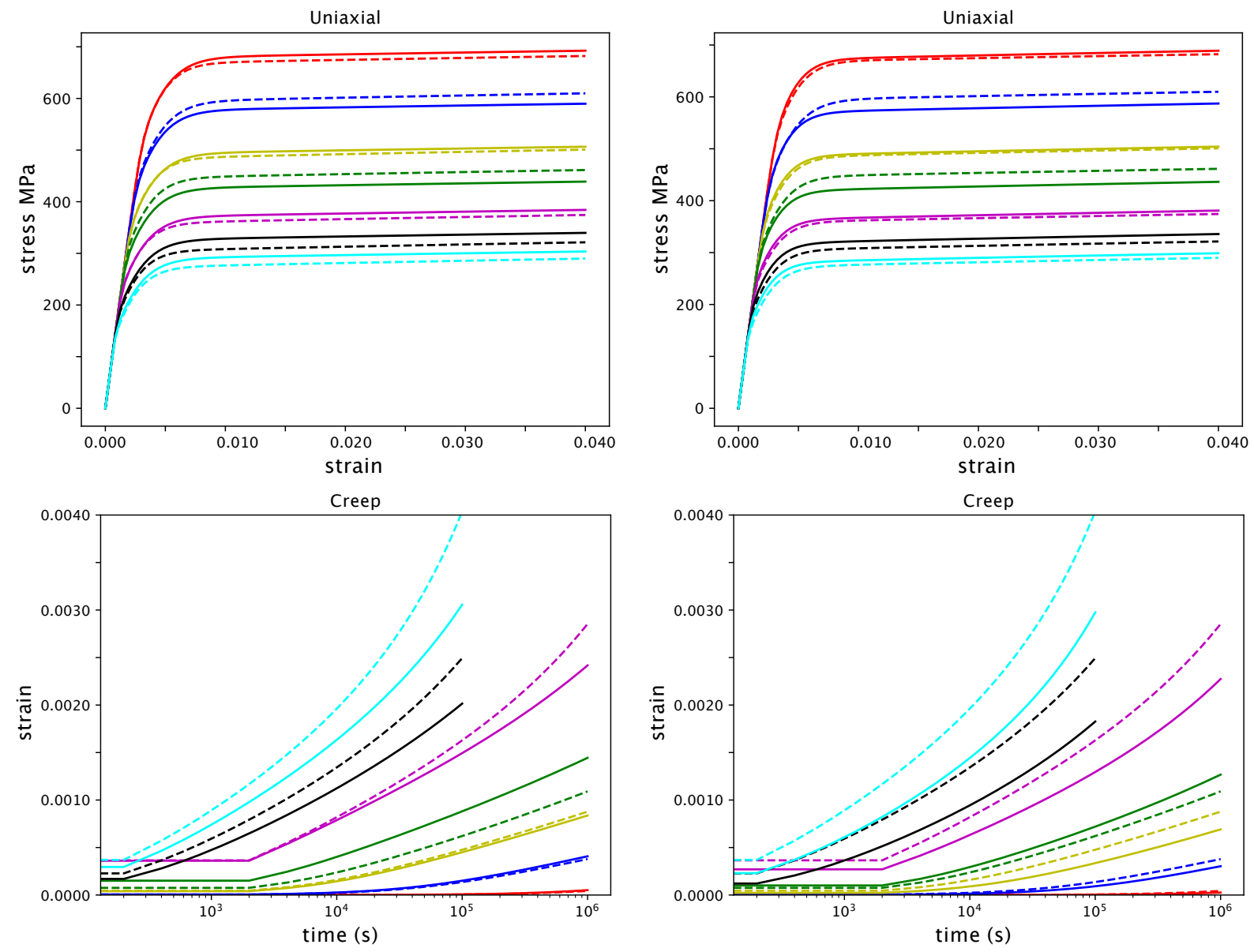

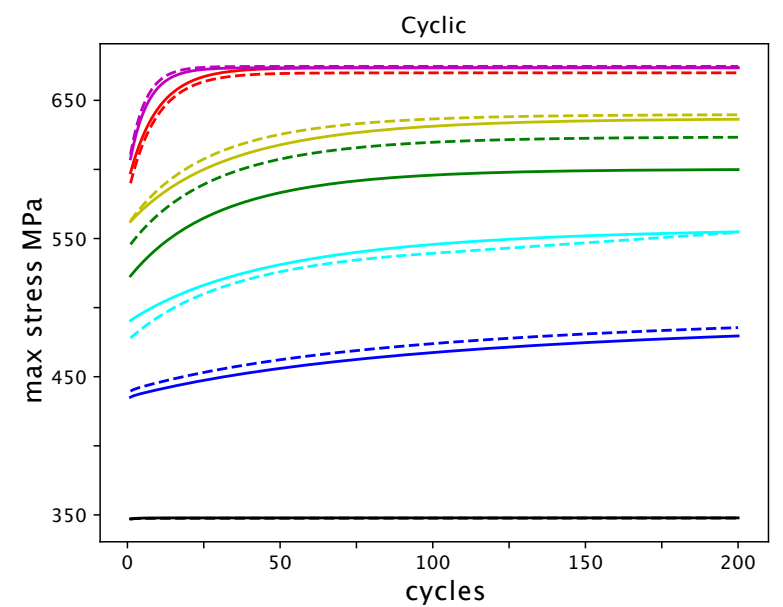

(a)

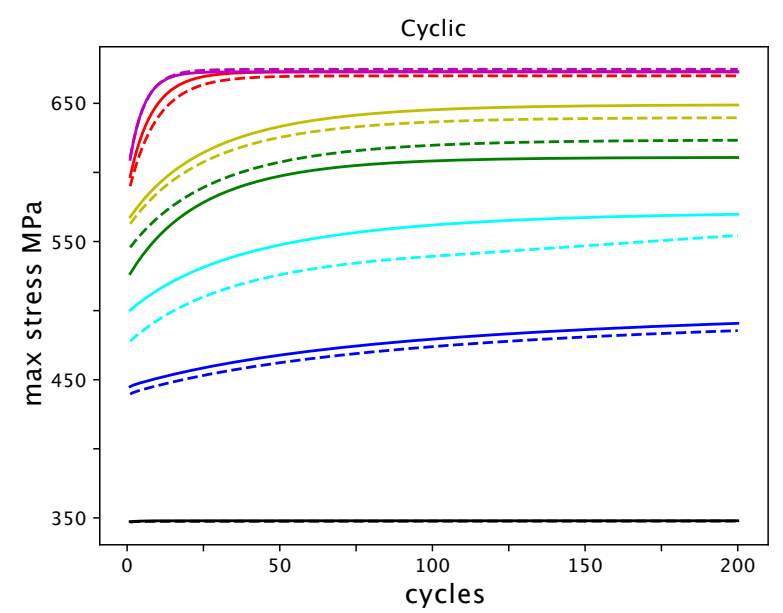

(b)

Figure 2.7: Comparison between the actual material responses (dashed lines) and their predicted responses (solid lines), with the posterior means obtained with prior mean of 0.3 (a) and 0.7 (b), respectively. The material response is recovered accurately. 

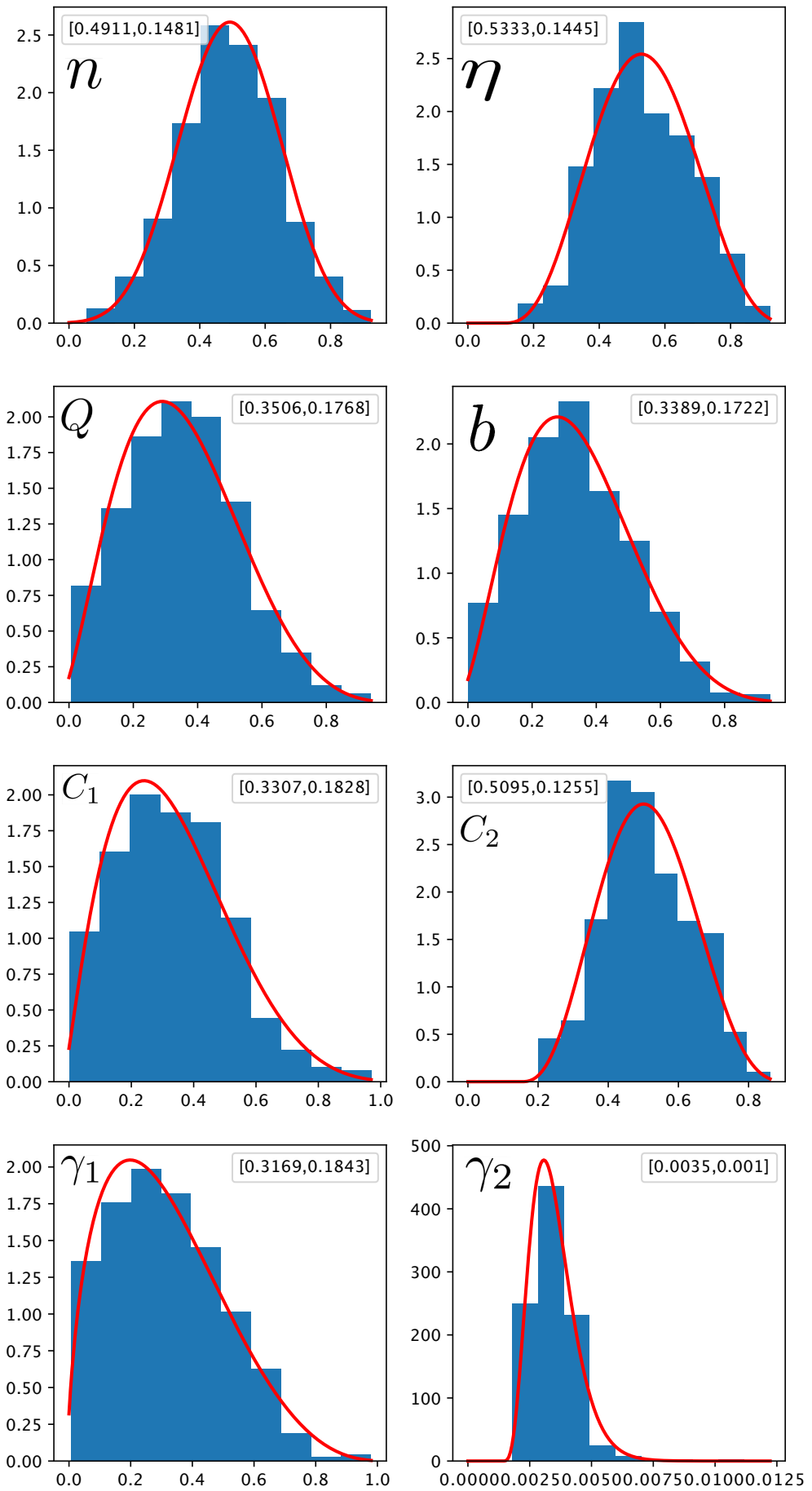

Figure 2.8: Posterior distributions as a fitted beta distribution (red) from the normalized histogram (blue) with truncated normal prior distributions having mean 0.3 and standard deviation of 0.2 for all the inelastic parameters of the cyclic model, with widened bounds for the kinematic hardening parameters. 


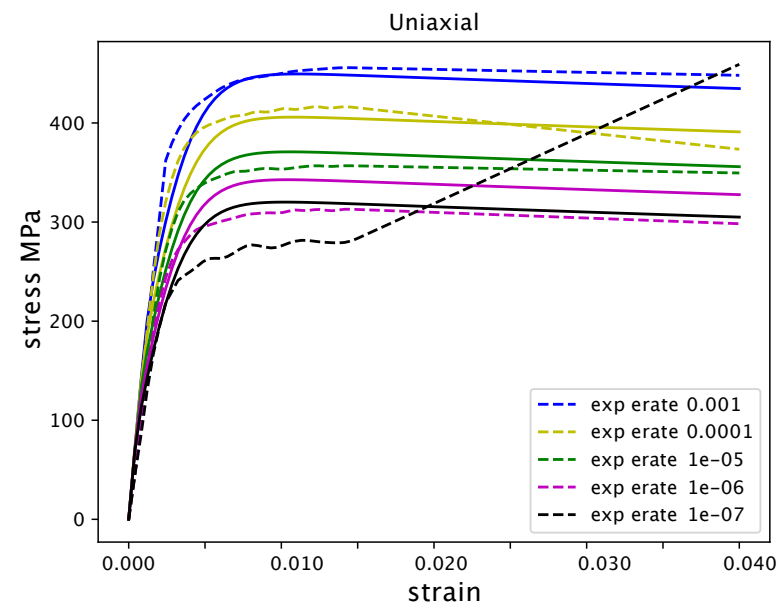

(a)

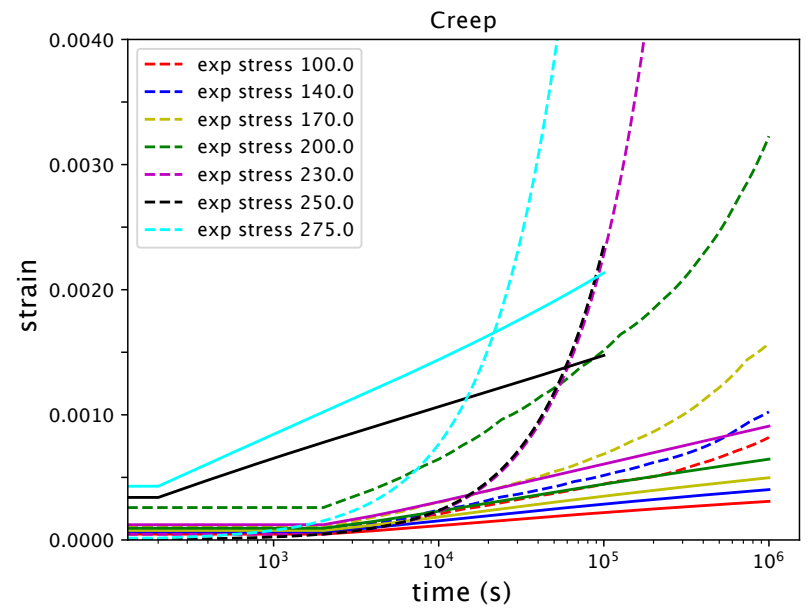

(b)

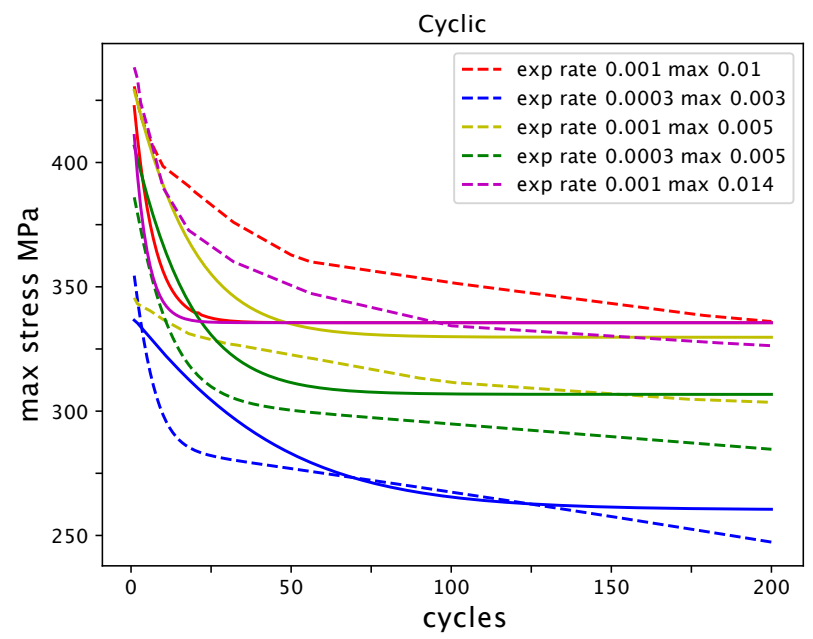

(c)

Figure 2.9: Calibrated model by fitting real experimental data for Grade 91 at $550{ }^{\circ} \mathrm{C}$ for uniaxial (a), creel (b), and (c) cyclic data. The dashed lines show the real experimental response while the solid lines are the predicted response. 
Table 2.4: Bounds for the initial calibration for the cyclic material model.

\begin{tabular}{cc}
\hline parameter & Bounds \\
\hline$n$ & {$[5,15]$} \\
$\eta$ & {$[100,950]$} \\
$Q$ & {$[-300,50]$} \\
$b$ & {$[3,10]$} \\
$C_{1}$ & {$\left[1.0 \times 10^{2}, 2.0 \times 10^{5}\right]$} \\
$C_{2}$ & {$\left[1.0 \times 10^{2}, 2.0 \times 10^{5}\right]$} \\
$\gamma_{1}$ & {$\left[1.0 \times 10^{2}, 2.0 \times 10^{5}\right]$} \\
$\gamma_{2}$ & {$\left[1.0 \times 10^{2}, 2.0 \times 10^{5}\right]$} \\
\hline
\end{tabular}

perimental data using the GA optimization algorithm. Tertiary creep is not observed because we have not considered damage in our model.

Bayesian MCMC We ran preliminary Bayesian MCMC analysis for real experimental data with two Markov chains having a population of 500 and 300 burn-in points. The standard deviation, $\xi$, in the likelihood is set to 200.0, 0.01, and 100.0 for tensile, creep, and cyclic experimental conditions, respectively. Figure 2.10 plots the posterior distributions for all eight inelastic parameters for the cyclic model, as obtained from Bayesian MCMC with real experimental Grade 91 data. These are preliminary results because the Markov chains did not converge, as clearly seen from the results for the kinematic hardening parameters $C_{1}$ and $\gamma_{1}$. The high correlation between the Chaboche backstress parameters could be one of the reasons for the premature convergence. Figure 2.11 compares the predicted responses with the parameter posterior means from the Bayesian analysis against the reference experimental data. The mean responses reasonably capture the true experimental behavior, indicating a feasible choice of the prior distributions. Additionally, in order to see the spread in the responses, we plotted by sampling eight points from the posterior distribution. Because the distributions are discrete, we selected these eight samples from the posterior such that they have different values for the parameter $\gamma_{1}$; i.e., we select eight equally spread points from a set of 53 unique values of $\gamma_{1}$, which also serves as the reference index for the other parameters. Figure 2.12 shows the spread in the predicted responses for the eight selected sample parameters from the posterior at three different test conditions for each of the tensile, creep, and cyclic boundary conditions. The spread in the response is lowest for the uniaxial and highest for the cyclic responses, and this captures the experimental response reasonably. This further indicates the insensitivity and the correlation among the hardening parameters. 

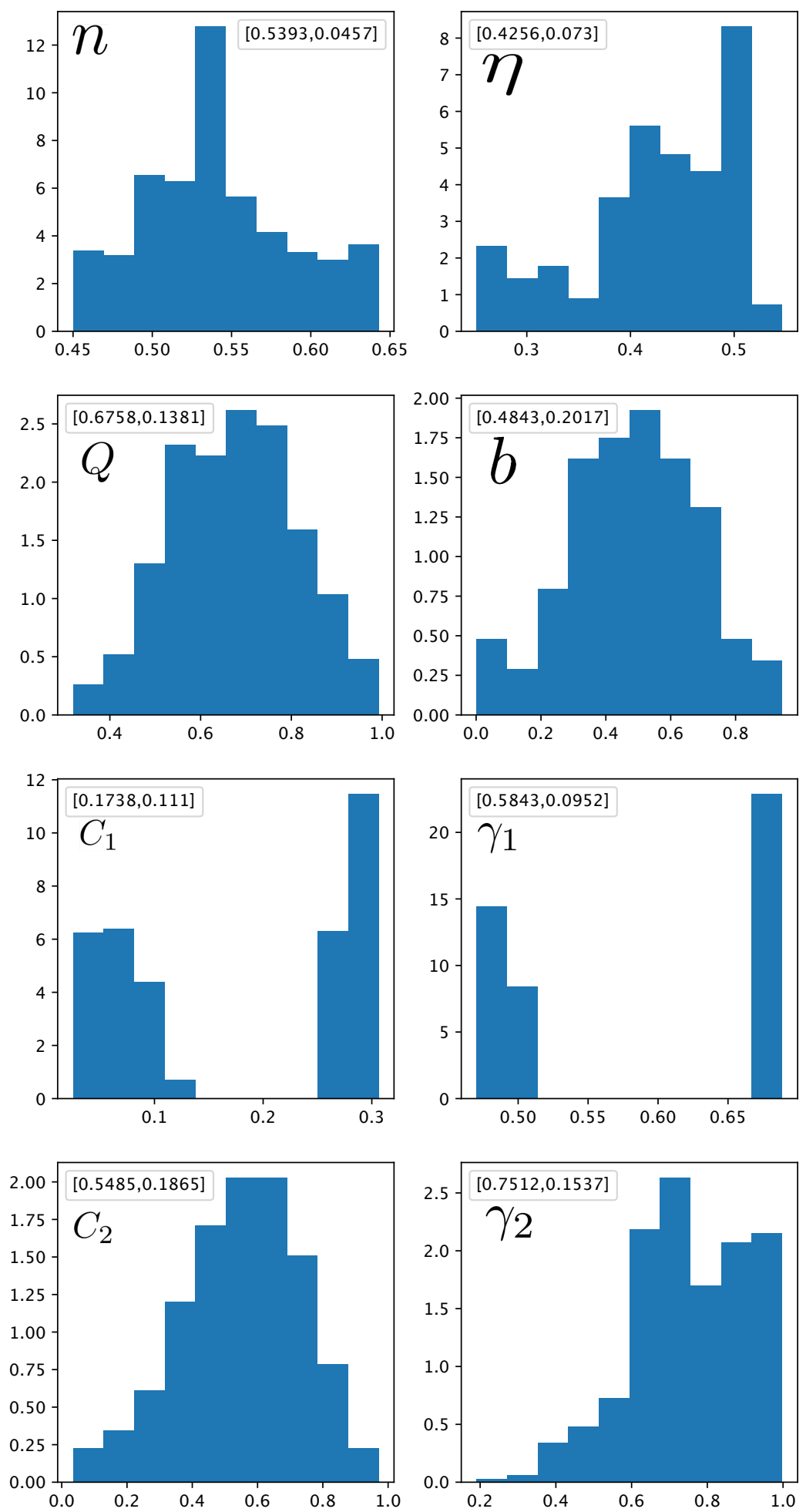

Figure 2.10: Posterior distributions as normalized histograms from the preliminary Bayesian MCMC for cyclic model, with real experimental Grade 91 data as reference, and with prior distributions being the initially calibrated model using the GA optimization. The numbers in the top corners represent the posterior means and standard deviations of the parameters. Clearly, the chains have not converged, likely because there is correlation between the kinematic hardening parameters. 


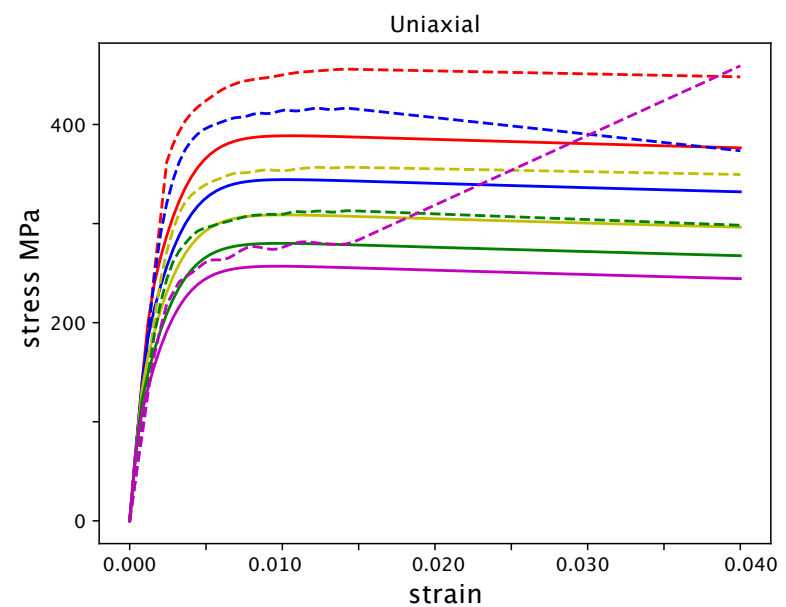

(a)

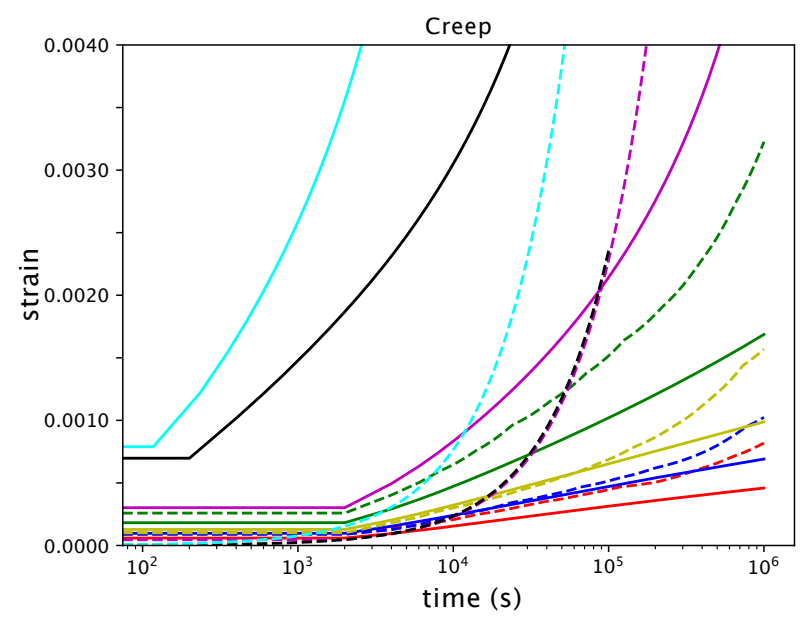

(b)

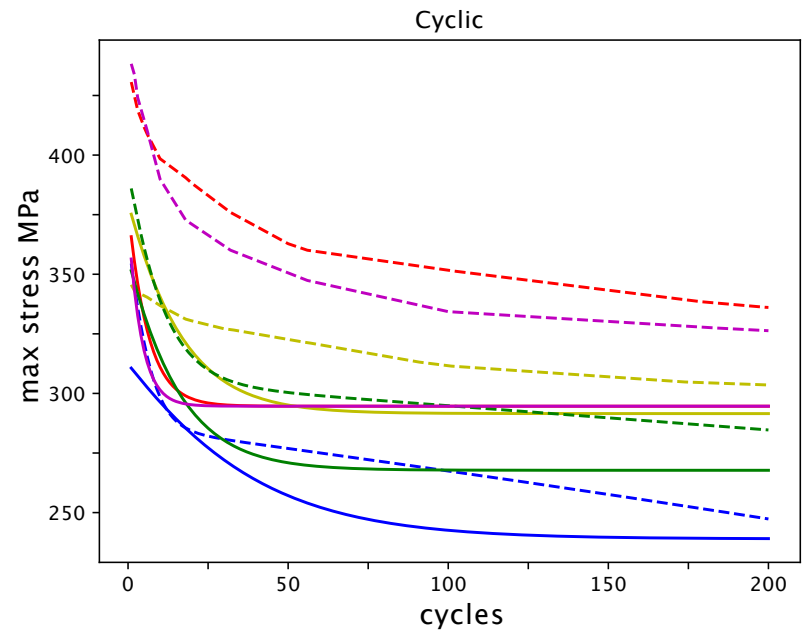

(c)

Figure 2.11: Predicted responses with the posterior means for (a) tensile, (b) creep, and (c) strain controlled cyclic boundary conditions. 

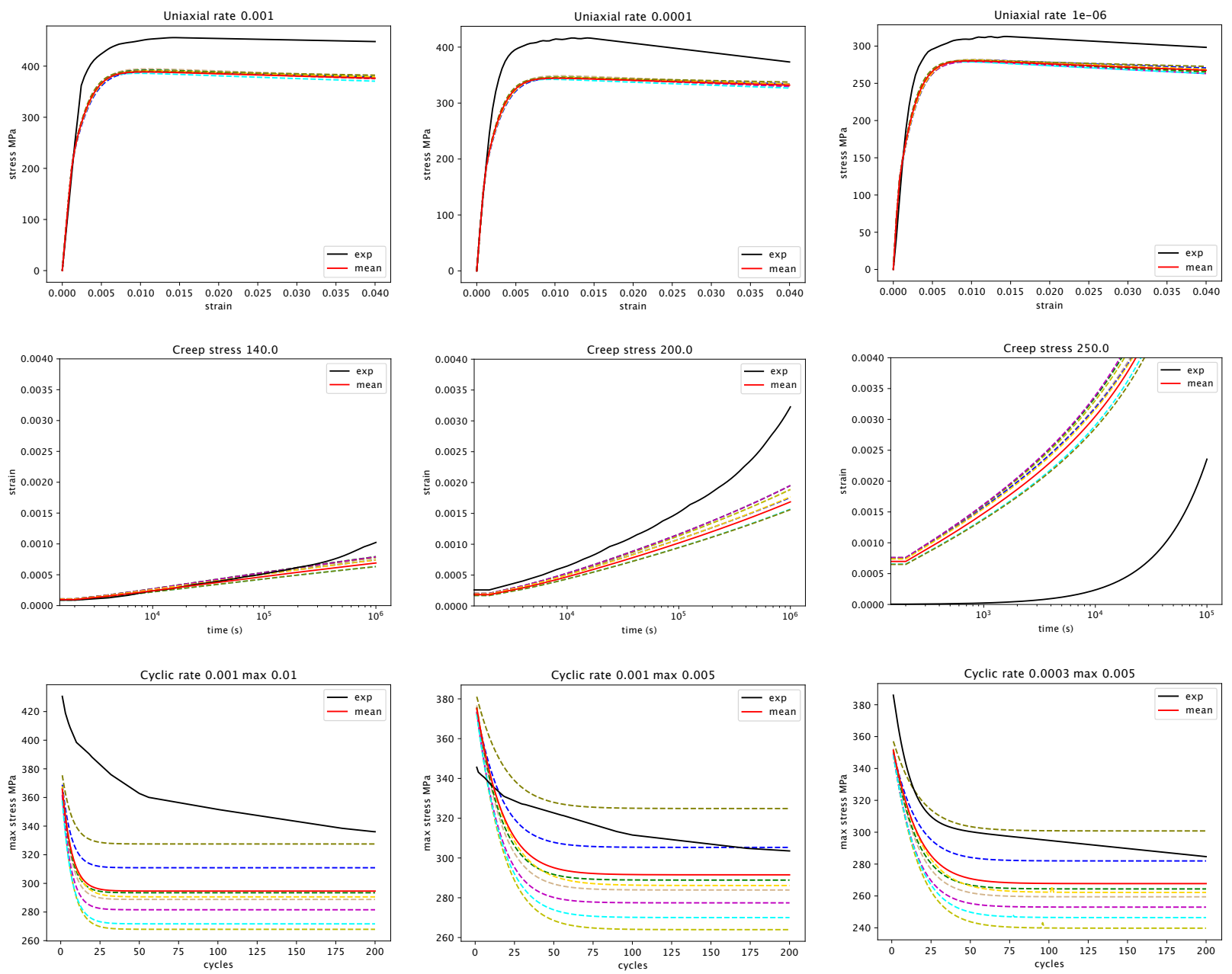

Figure 2.12: Predicted responses with eight sampled parameters from the posterior distributions at three different test conditions for each of the tensile (top), creep (middle), and strain controlled cyclic (bottom) boundary conditions. The respective test conditions are mentioned in the title of these plots. 


\subsection{Discussion}

\subsubsection{Uniaxial Model}

We tested different aspects of the Bayesian MCMC methodology with synthetic data and applied the insights obtained from those numerical studies to perform statistical modeling with real experimental Grade 91 data at 550 and $600{ }^{\circ} \mathrm{C}$.

Table 2.5: Prior and posterior means of the model parameters.

\begin{tabular}{cccccc}
\hline \multirow{2}{*}{ parameter } & \multicolumn{2}{c}{$550^{\circ} \mathrm{C}$} & & \multicolumn{2}{c}{$600^{\circ} \mathrm{C}$} \\
\cline { 2 - 3 } \cline { 5 - 6 } \cline { 5 - 6 } & prior mean & posterior mean & & prior mean & posterior mean \\
\hline$n$ & 10.81 & 11.0 & & 8.274 & 8.5 \\
$\eta$ & 844.61 & 832.0 & & 747.4 & 751.0 \\
$\sigma_{0}$ & 3.66 & 3.88 & & 3.550 & 3.5 \\
$Q$ & 106.03 & 107.0 & & 112.0 & 113.0 \\
$b$ & 45.43 & 48.0 & & 44.33 & 44.0 \\
$A$ & 924.911 & 927.0 & & 650.6 & 656.0 \\
$\zeta$ & 11.357 & 12.0 & & 10.71 & 12.0 \\
$\phi$ & 2.04 & 2.0 & & 2.04 & 2.0 \\
\hline
\end{tabular}

Table 2.5 compares the input prior means to their respective posterior means for all parameters of the uniaxial elasto-viscoplastic damage model. The closeness of prior and posterior means, along with the fact that the Markov chains are also converged (potential scale reduction factor close to unity), indicates the effectiveness of our starting location in the parameter space for the Bayesian MCMC analysis. This is an important consideration while performing Bayesian MCMC with such high dimensionality because there is always a chance that a Markov chain sticks at some region in the parameter space, thus giving non-converged posterior distributions. The hyperparameter values ( $\xi$ in the likelihood function) represent the spread that we want to capture through the posterior values. With the specified value of $\xi=200.0$ for the tension condition, the simulated tensile curves encompass the experimental data for most cases. Figure 2.4 shows a spread in the yield strength of around 50-100 MPa and a spread in the failure times of around two orders of magnitude. The spread in yield strength at $550^{\circ} \mathrm{C}$ reduces significantly with increasing strain rate, which is not the case for $600^{\circ} \mathrm{C}$ results, (a) and (c) in Figure 2.4.

\subsubsection{Cyclic Model}

We also performed preliminary statistical parameter modeling with a cyclic elasto-viscoplastic material model with kinematic hardening parameters using both synthetically generated reference data as well as real experimental data of Grade 91 at $550{ }^{\circ} \mathrm{C}$.

\section{Synthetic Experimental Data}

Figure 2.6 shows that the Bayesian MCMC accurately recovers true solutions for the parameters $n$ and $\eta$ from the synthetically generated reference data, as also observed with the uniaxial model, while the hardening parameters hardly change from their respective priors. Comparing plots in Figure 2.7, it appears that even with these estimations for the hardening parameters, the predicted responses are very close to the true solution. Increasing the bounds for these parameters clearly shows the efficacy of the Bayesian MCMC to accurately recover the true solution for the hardening parameters as well. 


\section{Real experimental data}

Table 2.6: Prior and posterior means of the model parameters for Grade $91 \mathrm{cyclic}$ model at $550{ }^{\circ} \mathrm{C}$.

\begin{tabular}{ccc}
\hline parameter & prior mean & posterior mean \\
& & \\
\hline$n$ & 0.5 & 0.5393 \\
$\eta$ & 0.387 & 0.4256 \\
$Q$ & 0.535 & 0.6758 \\
$b$ & 0.517 & 0.4843 \\
$C_{1}$ & 0.656 & 0.1738 \\
$\gamma_{1}$ & 0.587 & 0.5843 \\
$C_{2}$ & 0.569 & 0.5485 \\
$\gamma_{2}$ & 0.764 & 0.756 \\
\hline
\end{tabular}

Subsequently, we perform the Bayesian MCMC with the real experimental Grade 91 data at $550{ }^{\circ} \mathrm{C}$. The preliminary analysis does not result in a converged solution especially for the kinematic hardening parameters (discrete values for parameters $C_{1}$ and $\gamma_{1}$ in Figure 2.10) as also summarized in Table 2.6. This can be attributed to the choice of a short Markov chain (to lower computation time), as well as the strong correlation among the kinematic hardening parameters. Probable avenues to explore might be:

- Run with a larger Markov chain.

- Perform a sensitivity analysis to determine the relative sensitivity (and correlation) of the parameters to the likelihood function (especially the kinematic hardening parameters).

- Perform the Bayesian MCMC with multivariate distributions instead of independent univariate distribution for the kinematic hardening parameters.

- Fix two hardening parameters (preferably $C_{1}$ and $\gamma_{2}$ ) to those obtained from the calibration and vary the remaining two Chaboche parameters for the Bayesian MCMC. The underlying notion being, the desired spread in the response could be captured by a subset of the kinematic hardening parameters.

- Depending on the sensitivity analysis modify the likelihood function to better capture the effect of the hardening parameters.

The predicted responses from the posterior means, Figure 2.11 and their respective spread obtained by sampling parameters, Figure 2.12, reasonably captures the experimental response, thus highlighting the relative insensitivity of the kinematic hardening parameters.

\subsection{Conclusions}

\subsubsection{Summary}

This chapter describes a method for calibrating statistical models for empirical inelastic material models and applies the method to Grade 91 steel at different temperatures and different experimental conditions. The Bayesian MCMC methodology appears to be a promising technique in developing these statistical models. We considered monotonic and cyclic deformation. For the monotonic model, the Bayesian analysis converges and reasonable captures the observed spread in the experimental data. The posterior distributions with the real experimental data for the cyclic model should be further improved as a part of future work. Some of the key findings of this work can be summarized as: 
- An informed prior distribution significantly improves the chances for the Bayesian MCMC to find the accurate solution as compared to an uninformed prior.

- Not all the parameters are equally sensitive to the likelihood function, and hence a sensitivity analysis becomes crucial in determining the reliability of the predicted statistical model.

- The underlying data and the parameter bounds also play a critical role in determining the reliability of the methodology.

- A prior optimization to get a feasible starting point for the Bayesian is also highly efficient for the methodology to accurately obtain the true solution.

- Because the method is computationally expensive, we have developed the code using multiprocessing and performed the simulations using our high performance computing cluster.

\subsubsection{Future Work}

The Bayesian MCMC method did not accurately capture cyclic plasticity data. One factor here is the nondeterminism of the model parameter - a model with more than one backstress is mathematically not unique regardless of how much experimental data are available. There are infinite combinations of the backstress parameters that will all give the same kinematic hardening response. One avenue for future work is, then, developing a description of kinematic hardening that can provide a unique description of the experimental data. The simplest possibility is to fix the dynamic recovery parameters in the Chaboche model so as to force the different backstress terms to correspond to different regions of recovery. A better possibility would be to develop an improved representation of kinematic hardening that does not suffer from non-uniqueness.

In addition, the calibration method could be improved to produce better comparisons to the experimental scatter. Particularly for cyclic tests, acceleration strategies may be required to expedite the forward simulations through large numbers of load cycles. 


\section{Bibliography}

[1] Dianyin Hu, Rongqiao Wang, and Zhi Tao. Probabilistic design for turbine disk at high temperature. Aircraft Engineering and Aerospace Technology, 2011.

[2] Dianyin $\mathrm{Hu}$, Qihang Ma, Lihong Shang, Ye Gao, and Rongqiao Wang. Creep-fatigue behavior of turbine disc of superalloy gh720li at $650 \mathrm{c}$ and probabilistic creep-fatigue modeling. Materials Science and Engineering: A, 670:17-25, 2016.

[3] Felix Koelzow, Christian Kontermann, Klaus Helbig, and Dennis Jarmowski. Probabilistic lifetime assessment approach of $2 \%$-cr steel considering material and loading profile scatter. Journal of Engineering for Gas Turbines and Power, 142(1), 2020.

[4] Robert P Wei, David Masser, Hongwei Liu, and D Gary Harlow. Probabilistic considerations of creep crack growth. Materials Science and Engineering: A, 189(1-2):69-76, 1994.

[5] KM Nikbin, M Yatomi, K Wasmer, and GA Webster. Probabilistic analysis of creep crack initiation and growth in pipe components. International journal of pressure vessels and piping, 80(7-8):585-595, 2003.

[6] John H. Schaibly and Kurt E. Shuler. Study of the sensitivity of coupled reaction systems to uncertainties in rate coefficients. II Applications. Journal of Chemical Physics, 59(8):3879-3888, oct 1973.

[7] A F Saleeb, A S Gendy, and T E Wilt. Parameter-Estimation Algorithms for Characterizing a Class of Isotropic and Anisotropic Viscoplastic Material Models. Technical report, 2002.

[8] Rolf Mahnken, Magnus Johansson, and Kenneth Runesson. Parameter estimation for a viscoplastic damage model using a gradient-based optimization algorithm. Engineering Computations (Swansea, Wales), 15(6-7):925-955, 1998.

[9] O. T. Bruhns and D. K. Anding. On the simultaneous estimation of model parameters used in constitutive laws for inelastic material behaviour. International journal of plasticity, 15(12):1311-1340, 1999.

[10] A. Andrade-Campos, S. Thuillier, P. Pilvin, and F. Teixeira-Dias. On the determination of material parameters for internal variable thermoelastic-viscoplastic constitutive models. International Journal of Plasticity, 23(8):1349-1379, aug 2007.

[11] J. C. Gelin and O. Ghouati. An inverse method for determining viscoplastic properties of aluminium alloys. Journal of Materials Processing Tech., 45(1-4):435-440, 1994.

[12] A. F. Saleeb, T. E. Wilt, N. R. Al-Zoubi, and A. S. Gendy. An anisotropic viscoelastoplastic model for composites - Sensitivity analysis and parameter estimation. Composites Part B: Engineering, 34(1):2139, jan 2003.

[13] Aritra Chakraborty and Philip Eisenlohr. Evaluation of an inverse methodology for estimating constitutive parameters in face-centered cubic materials from single crystal indentations. European Journal of Mechanics, A/Solids, 66:114-124, nov 2017. 
[14] Jörgen Kajberg and Bengt Wikman. Viscoplastic parameter estimation by high strain-rate experiments and inverse modelling - Speckle measurements and high-speed photography. International Journal of Solids and Structures, 44(1):145-164, jan 2007.

[15] T. Massé and Y. Lejeail. Creep behaviour and failure modelling of modified 9Cr1Mo steel. In Nuclear Engineering and Design, volume 246, pages 220-232, may 2012.

[16] M. C. Messner, V.-T. Phan, and T.-L. Sham. A Unified Inelastic Constitutive Model for the Average Engineering Response of Grade 91 Steel. ASME International, jul 2018.

[17] M. C. Messner, V. T. Phan, and T. L. Sham. Evaluating and modeling rate sensitivity in advanced reactor structural materials: 316H, Gr. 91, and A617. International Journal of Pressure Vessels and Piping, 178, dec 2019.

[18] R. Sánchez-Martín, M. T. Pérez-Prado, J. Segurado, J. Bohlen, I. Gutiérrez-Urrutia, J. Llorca, and J. M. Molina-Aldareguia. Measuring the critical resolved shear stresses in $\mathrm{Mg}$ alloys by instrumented nanoindentation. Acta Materialia, 71:283-292, 2014.

[19] T. Seibert, J. Lehn, S. Schwan, and F. G. Kollmann. Identification of material parameters for inelastic constitutive models: Stochastic simulations for the analysis of deviations. Continuum Mechanics and Thermodynamics, 12(2):95-120, 2000.

[20] H. Rappel, L. A.A. Beex, L. Noels, and S. P.A. Bordas. Identifying elastoplastic parameters with Bayes' theorem considering output error, input error and model uncertainty. Probabilistic Engineering Mechanics, 55:28-41, jan 2019.

[21] Delphine D. Fitzenz, André Jalobeanu, and Stephen H. Hickman. Integrating laboratory creep compaction data with numerical fault models: A Bayesian framework. Journal of Geophysical Research, 112(B8):B08410, aug 2007.

[22] Sandeep Madireddy, Bhargava Sista, and Kumar Vemaganti. A Bayesian approach to selecting hyperelastic constitutive models of soft tissue. Computer Methods in Applied Mechanics and Engineering, 291:102-122, jul 2015.

[23] Jinhyuk Gang, Jooho Choi, Bonghee Lee, and Jinwon Joo. Material parameter identification of viscoplastic model for solder alloy in electronics package using Bayesian calibration. In Proceedings of the ASME Design Engineering Technical Conference, volume 3, pages 827-834, 2010.

[24] Jin Hyuk Gang, Dawn An, Jin Won Joo, and Joo Ho Choi. Uncertainty analysis of solder alloy material parameters estimation based on model calibration method. Microelectronics Reliability, 52(6):11281137, jun 2012.

[25] Erfan Asaadi and P. Stephan Heyns. A computational framework for Bayesian inference in plasticity models characterisation. Computer Methods in Applied Mechanics and Engineering, 321:455-481, jul 2017.

[26] Saikumar R. Yeratapally, Michael G. Glavicic, Christos Argyrakis, and Michael D. Sangid. Bayesian uncertainty quantification and propagation for validation of a microstructure sensitive model for prediction of fatigue crack initiation. Reliability Engineering and System Safety, 164(April 2016):110-123, 2017.

[27] Eliška Janouchová and Anna Kučerová. Bayesian inference of heterogeneous viscoplastic material parameters. Acta Polytechnica CTU Proceedings, 15:41-45, dec 2018. 
[28] Piotr Perzyna. Fundamental Problems in Viscoplasticity. Advances in Applied Mechanics, 9(C):243$377,1966$.

[29] J. L. Chaboche. A review of some plasticity and viscoplasticity constitutive theories. International Journal of Plasticity, 24(10):1642-1693, oct 2008.

[30] F. A. Leckie and D. R. Hayhurst. Constitutive equations for creep rupture. Acta Metallurgica, 25(9):1059-1070, 1977.

[31] P. E. Hadjidoukas, P. Angelikopoulos, D. Rossinelli, D. Alexeev, C. Papadimitriou, and P. Koumoutsakos. Bayesian uncertainty quantification and propagation for discrete element simulations of granular materials. Computer Methods in Applied Mechanics and Engineering, 282:218-238, dec 2014.

[32] John Salvatier, Thomas V. Wiecki, and Christopher Fonnesbeck. Probabilistic programming in Python using PyMC3. PeerJ Computer Science, 2(e55), 2016.

[33] Siddhartha Chib and Edward Greenberg. Understanding the metropolis-hastings algorithm. American Statistician, 49(4):327-335, 1995.

[34] Christophe Andrieu, Nando De Freitas, Arnaud Doucet, and Michael I. Jordan. An introduction to MCMC for machine learning. Machine Learning, 50(1-2):5-43, jan 2003.

[35] Masatsugu Yaguchi and Yukio Takahashi. Unified Inelastic Constitutive Model for Modified 9Cr-lMo Steel Incorporating Dynamic Strain Aging Effect. JSME International Journal Series A, 42(1):1-10, 1999.

[36] Masatsugu Yaguchi and Yukio Takahashi. Ratchetting of viscoplastic material with cyclic softening, part 1: Experiments on modified 9Cr-1Mo steel. International Journal of Plasticity, 21(1):43-65, jan 2005 .

[37] B. K. Choudhary and E. Isaac Samuel. Creep behaviour of modified 9Cr-1Mo ferritic steel. Journal of Nuclear Materials, 412(1):82-89, may 2011.

[38] S. Latha, M. Nandagopal, S. Panneer Selvi, K. Laha, and M. D. Mathew. Tensile and creep behaviour of modified 9Cr-1Mo steel cladding tube for fast reactor using metallic fuel. In Procedia Engineering, volume 86, pages 71-79. Elsevier Ltd, 2014.

[39] Kouichi Maruyama, Junya Nakamura, Kyosuke Yoshimi, and Yuji Nagae. Evaluation of long-term creep rupture life of Gr. 91 steel by analysis of on-going creep curves. In Proceedings from the Eighth International Conference on Advances in Materials Technoglogy for Fossil Power Plants, pages 467478, 2016.

[40] R W Swindeman. Cyclic stress-strain-time response of a 9Cr-1Mo-V-Nb pressure vessel steel at high temperature. In Low Cycle Fatigue. ASTM International, 1988.

[41] K. Kimura, H. Kushima, and K. Sawada. Long-term creep deformation property of modified 9Cr-1Mo steel. Materials Science and Engineering A, 510-511(C):58-63, jun 2009. 


\section{MICROSTRUCTURE-BASED REDUCED ORDER MODEL FOR ENGINEERING-SCALE CONSTITUTIVE RESPONSE}

While the previous section focused on the presentation of an approach to quantify the variability in mechanical performance of a structure resulting from distinct choices of materials systems - although nominally these are considered as similar - the current section aims at introducing an engineering scale constitutive model that relates the creep response of Grade 91 to its microstructure. This will be described in terms of dislocation densities in the cell interiors, the cell walls, and of the overall MX precipitate phase fraction, which is known to have significant impact on the materials transient and steady-state creep response [1]. This initial effort will therefore allow for a quantification of the effect of microstructure variability on the performance of an engineering scale component. Provided this model's performance is acceptable, the longer term prospects of this work are to derive a unified model that can relate the differences in mechanical response between nominally similar materials, but with different pedigrees (e.g., P91, T91) to the initial microstructure of the medium.

The approach proposed relies, first, on the use of a crystal-plasticity (CP) based mechanistic constitutive model. This model is calibrated and validated with experimental Grade 91 steel creep test data. Here, only experimental data with consistent pedigree are used. The model is then applied to quantify the effect of variations in the MX precipitate content and dislocation content on primary and secondary creep response of P91. Leveraging the CP-based model, a database of synthetic creep response is generated. This database is then mined to generate a surrogate model (SM), which is known as LAROMANCE Gr91. This is a model that can be used in practical engineering-scale calculations, and because it is based on an underlying grain-scale model, is sensitive to the material's microstructure.

In what follows, the CP-based constitutive model is described in Section 3.1. The creep test simulation database generation methods are described in Section 3.2. The formulation of the SM from a series of polynomial regression models is described in Section 3.3. The demonstration and validation of the SM under time-varying loading conditions is given in Section 3.4.

\subsection{Crystal Plasticity Constitutive Model}

The physics-based constitutive model, which quantifies the effects of stress, temperature and microstructure on the activation of distinct deformation mechanisms to predict the thermal creep behavior of Grade 91 alloy, is presented in what follows. This work is based on the constitutive models initially developed in previous studies [2,3]. This single crystal constitutive law is embedded in a mean-field visco-plastic selfconsistent (VPSC) framework thereby allowing for the prediction of the effective mechanical response of a polycrystalline aggregate from the description $[4,5]$. Using a small strain formulation, the total plastic strain rate is expressed as:

$$
\dot{\varepsilon}^{p}=\dot{\varepsilon}^{d}+\dot{\varepsilon}^{\text {coble }}
$$

where $\dot{\varepsilon}^{d}$ and $\dot{\varepsilon}^{\text {coble }}$ refer to the plastic deformation accumulated through dislocation motion and diffusion process (e.g., Coble creep), respectively. The strain rates due to dislocation motion can be written as the sum 
of the mean shear rates, $\overline{\dot{\gamma}}$, over all active systems in the grain:

$$
\dot{\varepsilon}^{d}=\sum_{s} m_{i j}^{s} \dot{\gamma}^{s}
$$

where $m^{s}$ is the symmetric part of the Schmid tensor. In effective medium models, such as the VPSC framework, the strain rate and stress within each grain are homogeneous. In practice, though, stress distribution within each grain is heterogeneous as a consequence of the presence of defects which are non-uniformly distributed within the medium. To capture this, every grain can be theoretically discretized into sub-grain points, each with distinct internal stresses, and the stress and strain rates will be different depending on local dislocation distributions. Following Wang et al. [6], the heterogeneous response within each grain is represented in a statistical fashion via internal stress distribution. Accordingly, the mean shear rate of the slip system $s$ in the grain domain is expressed as:

$$
\overline{\dot{\gamma}}^{s}=\int_{-\infty}^{\infty} \dot{\gamma}^{s}\left(\tau^{s}\right) P\left(\tau^{s}-\bar{\tau}^{s}\right) d \tau^{s}
$$

where $\dot{\gamma}^{s}$ is the shear rate of one sub-material point, $\tau^{s}$, is the local resolved shear stress. $\bar{\tau}^{s}=\sigma: m^{s}$ denotes the mean resolved shear stress in one grain where $\sigma$ is the deviatoric stress tensor. The probability distribution function $P\left(\tau^{s}-\bar{\tau}^{s}\right)$, representing the volume fraction of sub-material points with $\tau^{s}$, is represented by the Gaussian distribution as,

$$
P\left(\tau^{s}-\bar{\tau}^{s}\right)=\frac{1}{\sqrt{2 \pi V}} \exp \left(-\frac{\left(\tau^{s}-\bar{\tau}^{s}\right)^{2}}{2 V^{2}}\right)
$$

where $V$ is the variance of the resolved shear stress, and it depends on the dislocation density. For the sake of simplicity, $V$ is assumed to be constant throughout the creep simulations, even though the dislocation density will decrease during creep.

The local shear rate on system $s$ due to dislocation motion can be expressed using the Orowan equation as,

$$
\dot{\gamma}^{s}=\rho_{\text {cell }}^{s} b^{s} v^{s} . \operatorname{sign}\left(\tau^{s}\right)
$$

where $b^{s}$ is the magnitude of the Burgers vector. $v^{s}$ is the mean dislocation velocity, which can be determined from the mean spacing between obstacles (dislocation mean free path, $\lambda^{s}$ ) and the time a dislocation spends traveling between obstacles, given by the sum of the waiting time at obstacles $\left(t_{w}^{s}\right)$ and the travel time within the interspacing $\left(t_{t}^{s}\right)$ :

$$
v^{s}=\frac{\lambda^{s}}{t_{w}^{s}+t_{t}^{s}}
$$

Within each sub-grain, multiple types of obstacles like precipitates and dislocation junctions may co-exist and control dislocation motion. In P91 alloy, within subgrains, quasi spherical MX precipitates are almost uniformly distributed. At the same time, the rod-like M23C6 precipitates are located mainly at the sub-grain and grain boundaries. Thus, within subgrains, dislocation motion will be controlled by the MX precipitates and other dislocations. Specifically, the presence of multiple obstacles leads to reduction in the mean free path and increase in waiting time. In this work, the effective mean free path $\lambda^{s}$ is assumed to be the geometric mean of the interspacing for individual obstacles:

$$
\frac{1}{\lambda^{s}}=\frac{1}{\lambda_{\rho, \text { cell }}^{s}}+\frac{1}{\lambda_{M X}^{s}}
$$


where $\lambda_{\rho, \text { cell }}^{s}$ and $\lambda_{M X}^{s}$ denote the dislocation mean free path for dislocation obstacles and MX precipitates, respectively. The mean dislocation interspacing associated with dislocation-dislocation interactions can be given as [7]:

$$
\frac{1}{\lambda_{\rho, \text { cell }}^{s}}=\sqrt{\sum_{s} \bar{\alpha}^{s s^{\prime}} \rho_{\text {cell }}^{s^{\prime}}}
$$

where $\bar{\alpha}^{s s^{\prime}}$ refers to the latent hardening matrix. Following the geometric configuration of obstacles on a plane, the mean interspacing for MX precipitates is written as,

$$
\frac{1}{\lambda_{M X}^{s}}=h_{M X} \sqrt{N_{M X} d_{M X}}
$$

where $h_{M X}, N_{M X}$ and $d_{M X}$ are the trapping coefficient, number density and size of MX precipitates, respectively.

Stored dislocations can overcome the obstacles either by thermally activated glide, $t_{w, i, g}^{s}$ (e.g., junction unzipping and Orowan bypass) and/or climb-assisted glide process, $t_{w, i, c g}^{s}[8,9]$. These two mechanisms can occur simultaneously, so the total waiting time can be reduced. With this, the waiting time for each obstacle type $i$ ( $=\rho$ for dislocations, and MX for precipitates) can be written as,

$$
\frac{1}{t_{w, i}^{s}}=\frac{1}{t_{w, i, g}^{s}}+\frac{1}{f t_{w, i, c g}^{s}}
$$

where a factor $f(>1)$ is introduced to account for the additional waiting time required to detach the dislocations from obstacles after the climb-over process. The mean waiting time of slip system $\mathrm{s}$ when both the obstacles are considered is given by the average of $t_{w, \rho}^{s}$ and $t_{w, M X}^{s}$ as,

$$
t_{w}^{s}=P_{\rho} t_{w, \rho}^{s}+\left(1-P_{\rho}\right) t_{w, M X}^{s}
$$

where $P_{\rho}$ is the probability that a dislocation encounters another dislocation and $1-P_{\rho}$ that it encounters a MX precipitate.

\subsubsection{Waiting Time for Thermally Activated Glide}

The bypass of both dislocation and MX precipitate type obstacles can be considered as a thermally activated process either by junction unzipping and Orowan mechanisms. Thus, the waiting time can be described using a Kocks-type activation enthalpy law [10] as:

$$
t_{w, g, i}^{s}=\frac{1}{v_{i}^{s}} \exp \left(\frac{\Delta G_{0, i}}{k T}\left(1-\left(\frac{\left|\tau^{s}\right|}{\tau_{c}^{s}}\right)^{p}\right)^{q}\right) \quad \text { if }\left|\tau^{s}\right|<\tau_{c}^{s} 0 \text { if }\left|\tau^{s}\right| \geq \tau_{c}^{s}
$$

where $\Delta G_{0, i}$ is the thermal activation energy without any external stress, $k$ is Boltzmann constant. and $T$ is the absolute temperature. $p(0<p \leq 1)$ and $q(0<q \leq 1)$ are exponent parameters related to the shape of the obstacles resistance profile [10]. $v_{i}^{s}$ is the attack frequency. $\tau_{c}^{s}$ refers to the Critical Resolved Shear Stress (CRSS). Apart from the intrinsic frictional resistance $\tau_{0}^{s}$, the net effect of all strengthening mechanisms can be expressed via use of a non-linear superposition law. The strengthening sources accounted for in this work include precipitates, clusters, solute pinning, and the dislocations in the cell wall (through long-range stress field). Hence, we have:

$$
\tau_{c}^{s}=\tau_{0}^{s}+\left(\tau_{c w}^{n}+\tau_{P}^{n}\right)^{1 / n}
$$

with $\tau_{c w}=\mu b^{s} \sqrt{\sum_{s} \bar{\alpha}^{s s^{\prime}} \rho_{c w}^{s^{\prime}}}$ denoting cell wall-induced hardening, and $\tau_{P}$ denotes strengthening due to precipitates. 


\subsubsection{Waiting Time For Climb Assisted Glide}

The waiting time for climb can be determined from the mean climb velocity of edge dislocations and average distance to climb before bypass. Therefore, the average waiting time for edge dislocations is expressed as,

$$
\frac{1}{t_{w, i, c g}^{s}}=R_{e} \frac{\left|v_{c}^{s}\right|}{d_{i}^{c}}
$$

where $R_{e}$ denotes the proportion that edge dislocations contribute to the climb process. $d_{i}^{c}$ is the distance to climb to bypass obstacles. Following Wen et al. [3], the climb velocity $v_{c}^{s}$ is expressed as,

$$
v_{c}^{s}=\frac{2 \pi D_{v}\left[C_{v}^{0}-C_{v}^{0} \exp \left(\frac{-f_{c}^{s} \Omega}{k T b}\right)\right]}{b \ln \left(\frac{r_{\infty}}{r_{d}}\right)}
$$

where $\Omega \approx b^{3}$ is the atomic volume. $f_{c}^{s}$ is the climb component of Peach-Koehler force. $D_{v}$ is the vacancy diffusivity, and it is calculated by:

$$
D_{v}=D_{v}^{0} \exp \left(\frac{-E_{m}^{v}}{k T}\right)
$$

where $E_{m}^{v}$ is the vacancy migration energy. $C_{v}^{0}$ is the equilibrium vacancy concentration at temperature $\mathrm{T}$ in the bulk of the crystal, and it is given by,

$$
C_{v}^{0}=\exp \left(\frac{S_{f}^{v}}{k}\right) \exp \left(\frac{-E_{f}^{v}}{k T}\right)
$$

where $S_{f}^{v}$ and $E_{f}^{v}$ are the vacancy formation entropy and energy, respectively, and it is written as a function of temperature as,

$$
\begin{gathered}
E_{f}^{v}=g_{o}-g_{2} T^{2}-2 g_{3} T^{3} \\
S_{f}^{v}=-g_{1}-2 g_{2} T-.3 g_{3} T^{2}
\end{gathered}
$$

The $\mathrm{g}_{\mathrm{x}}$ coefficients are listed in Table 3.1. The terms $r_{d}$ and $r_{\infty}$ in climb velocity equation denote the radii of inner and outer boundaries for the cylindrical control volume defined around the dislocation line.

\subsubsection{Coble Creep}

Besides dislocation motion, the diffusion process may also contribute to plastic deformation in polycrystals via migration of point defects along grain boundaries and within grain interiors. This mechanism usually takes place at high-temperature and low or moderate stresses and dictates a creep regime with stress exponent $\approx 1$. In the present work, grain boundaries are not represented explicitly, and grain boundary related material properties are known for the steel alloy system considered. Thus, Coble creep is written in simple form as,

$$
\dot{\varepsilon}_{i j}^{\text {coble }}=\frac{A^{\text {coble }} S_{i j}}{T} \exp \left(-\frac{Q_{g b}}{k T}\right)
$$

where $A^{\text {coble }}$ and $Q_{g b}$ are effective parameters accounting for all point defects and can be obtained through the fitting to the experimental data. $S_{i j}$ denotes the $i j$ component of the deviatoric stress tensor. 


\subsubsection{Dislocation Density Evolution}

The dislocation density evolution law is essential to capture transient in the creep rate. The dislocation density affects the shear rate on each slip system. Besides, total dislocation density is required to the determine the variance of the Gaussian internal stress distribution. Notice that only the dislocations in the cell can move and contribute to dissipation. The evolution of the dislocation density in the cell is expressed as:

$$
\dot{\rho}_{c e l l}^{s}=\dot{\rho}_{c e l l, g}^{s,+}-\dot{\rho}_{c e l l, a}^{s,-}-\dot{\rho}_{c e l l, t r a p}^{s,-}
$$

where $\dot{\rho}_{\text {cell,g }, s}^{s,+}, \dot{\rho}_{\text {cell,a, }}^{s,-}$ and $\dot{\rho}_{\text {cell,trap }}^{s,-}$ denotes the dislocation generation, dynamic recovery and trapping at the subgrain boundaries. The dislocation generation rate is associated with the area swept by the moving dislocations. The term $\dot{\rho}_{c e l l, g}^{s,+}$ can be expressed as $[10,11]$ :

$$
\dot{\rho}_{c e l l, g}^{s,+}=\frac{k_{1}}{b \lambda^{s}}\left|\overline{\dot{\gamma}}^{s}\right|
$$

where $\frac{\lambda^{s}}{k_{1}}$ is the effective mean free path. Dynamic recovery involves several mechanisms, such as cross-slip and climb, that allow the dislocation to move to another slip plane and annihilate with dislocations with opposite Burger vector. Estrin [12] proposed a general expression of the dynamic recovery rate:

$$
\dot{\rho}_{\text {cell }, a}^{s,-}=k_{2}\left(\frac{\dot{\varepsilon}_{0}}{\dot{\dot{\varepsilon}}}\right)^{\frac{1}{n_{0}}} \rho_{\text {cell }}^{s}\left|\overline{\dot{\gamma}}^{s}\right|
$$

where $\dot{\varepsilon}_{0}$ is a reference strain rate. Estrin suggested that the parameter $n_{0}$ should be associated with the dominant mechanism [12]. Its value should be between 3 and 5 if it is a climb dominated process, or higher otherwise. Dislocation trapping rate at the subgrain boundaries is related to the subgrain size $\lambda_{s g}$ :

$$
\dot{\rho}_{\text {cell,trap }}^{s,-}=\frac{k_{3}}{\lambda_{s g}}\left|\overline{\dot{\gamma}}^{s}\right|
$$

The trapped dislocations will essentially become part of the wall structure. Meanwhile, the dislocations in the cell wall will also annihilate. Thus, the rate of $\rho_{c w}^{s}$ can be written as:

$$
\dot{\rho}_{c w}^{s}=\dot{\rho}_{c e l l, t r a p}^{s,-}-\dot{\rho}_{c w, a}^{s,-}
$$

Dislocation annihilation in the subgrain boundaries is complex and its mechanism for the Fe-Cr alloy is not fully understood. For the sake of simplicity, the annihilation rate is given here as follows:

$$
\dot{\rho}_{c w, a}^{s,-}=k_{4}\left|v_{c}^{s}\right|\left(\rho_{c w}^{s}\right)^{3 / 2}
$$

The parameters $k_{1}, k_{2}, k_{3}$ and $k_{4}$ are material constants.

\subsubsection{Creep Simulations of P91 Alloy}

Thermal creep simulations of Grade 91 alloy are performed using the affine-VPSC formulation for four different temperatures - namely, 823K, $873 \mathrm{~K}, 923 \mathrm{~K}$ and $973 \mathrm{~K}$, for different stresses. The polycrystal model parameters are calibrated against the experimental results $[13,14]$. The following six experimental cases were used for calibration: $823 \mathrm{~K}$ and $200 \mathrm{MPa}, 873 \mathrm{~K}$ and $120 \mathrm{MPa}, 873 \mathrm{~K}$ and $200 \mathrm{MPa}, 923 \mathrm{~K}$ and $125 \mathrm{MPa}$, $973 \mathrm{~K}$ and $50 \mathrm{MPa}$, and $973 \mathrm{~K}$ and $150 \mathrm{MPa}$. The remaining cases, a total of 16, are predicted and validated against the experiment. The model material is assumed to be heat-treated; thus, the texture is approximated by 50 randomly oriented grains, as shown in Figure 3.1(a). Following the work of Wen et al. [2], we choose the initial values of $\rho_{c e l l}^{s}$ and $\rho_{c w}^{s}$ for each system to be $4 \times 10^{12} \mathrm{~m}^{-2}$ and $1 \times 10^{13} \mathrm{~m}^{-2}$, respectively. Similarly, 
the $N_{M X}$ and $d_{M X}$ are set to be $3 \times 10^{20} \mathrm{~m}^{-3}$ and $37 \mathrm{~nm}$, respectively. The predicted and calibrated model parameters are listed in Table 2.1.

The creep behavior of Grade 91 alloy predicted by the VPSC model for the four temperature cases is shown in Figure 3.1 (b)-(h), along with the experimental data. It clearly shows that the polycrystal model captures the thermal creep behavior accurately for all stress and temperature cases. The experimental data that include time-resolved creep rates (d-h) also include tertiary creep, i.e. damage, in some cases, e.g. (g). The increase in creep rate associated with tertiary creep is not addressed by the model and is not matched by the model. Note that the data at lower stresses and temperatures $(873$ and $823 \mathrm{~K})[15,16]$ were for the material T91, not P91. The data at $873 \mathrm{~K}$ and $200 \mathrm{MPa}$ for T91 were used to calibrate the Coble creep parameters. Coble creep is primarily a function of diffusivity thus, it is controlled by chemistry. From this, only small differences in the Coble creep properties of P91 and T91 are expected. 
Table 3.1: Calibrated constitutive model parameter values for P91 steel.

\begin{tabular}{|c|c|c|c|}
\hline Parameters & Values & Parameters & Values \\
\hline $\begin{array}{c}b \text { (magnitude of Burgers } \\
\text { vector) }\end{array}$ & $2.48 \cdot 10^{-10} \mathrm{~m}$ & $\begin{array}{c}\alpha_{0}^{S S \prime} \text { (saturation dislocation- } \\
\text { dislocation interaction) }\end{array}$ & $\begin{array}{l}0.7\left(s=s^{\prime}\right) \\
0.05\left(s \neq s^{\prime}\right)\end{array}$ \\
\hline$\mu$ (shear modulus) & $\begin{array}{r}103572 \mathrm{MPa} \\
-T \cdot 48 \mathrm{MPa} / \mathrm{K} \\
\end{array}$ & $\begin{array}{c}n_{0} \text { (annihilation strain rate } \\
\text { sensitivity) }\end{array}$ & 3.5 \\
\hline$\tau_{0}$ (friction stress) & $0 \mathrm{MPa}$ & $\dot{\varepsilon}_{0}($ reference strain rate $)$ & $1 \mathrm{~s}^{-1}$ \\
\hline $\begin{array}{c}N_{M X}(\mathrm{MX} \text { precipitate } \\
\text { number density) }\end{array}$ & $3 \times 10^{20} \mathrm{~m}^{-3}$ & \multirow{2}{*}{$\begin{array}{c}\tau_{M X} \text { (hardening contribution of } \\
\text { MX precipitates) }\end{array}$} & $\begin{array}{l}393 \mathrm{MPa} \text { at } 823 \mathrm{~K} \\
359 \mathrm{MPa} \text { at } 873 \mathrm{~K}\end{array}$ \\
\hline$\lambda_{s g}$ (sub-grain size) & $0.5 \times 10^{-6} \mathrm{~m}$ & & $\begin{array}{l}322 \mathrm{MPa} \text { at } 923 \mathrm{~K} \\
306 \mathrm{MPa} \text { at } 973 \mathrm{~K}\end{array}$ \\
\hline $\begin{array}{c}d_{M X} \text { (diameter of MX } \\
\text { precipitates })\end{array}$ & $37 \mathrm{~nm}$ & $\begin{array}{c}h_{M X} \text { (trapping coefficient for MX } \\
\text { precipitates) }\end{array}$ & 1 \\
\hline $\begin{array}{c}\tau_{h} \text { (hardening contribution } \\
\text { from solute pinning and } \\
\text { precipitation) }\end{array}$ & $\begin{array}{c}574.6 \mathrm{MPa} \\
-T \cdot 0.2 \mathrm{MPa} / \mathrm{K}\end{array}$ & $\begin{array}{c}v_{G, M X} \text { (attack frequency for } \mathrm{MX} \\
\text { precipitate obstacle) }\end{array}$ & $1.2 \times 10^{10} \mathrm{~s}^{-1}$ \\
\hline$\eta$ (scaling parameter) & $2.6 \cdot 10^{-6} \mathrm{MPa} / \mathrm{m}$ & $m$ (exponent factor) & 2.0 \\
\hline $\begin{array}{c}\Delta G_{0, \rho} \text { (zero-stress } \\
\text { activation energy for } \\
\text { dislocations) }\end{array}$ & $2.8 \mathrm{eV}$ & $\begin{array}{l}V \text { (resolved shear stress } \\
\text { variance) }\end{array}$ & $1000 \mathrm{MPa}^{2}$ \\
\hline $\begin{array}{c}\Delta G_{0, M X} \text { (zero-stress } \\
\text { activation energy for } \mathrm{MX} \\
\text { precipitates) }\end{array}$ & $7 \mathrm{eV}$ & $\begin{array}{l}R_{e} \text { (proportion of edge } \\
\text { dislocations) }\end{array}$ & $10 \%$ \\
\hline$p$ (exponent parameter) & 0.7 & $\begin{array}{l}\chi \text { (pre-factor linking binding } \\
\text { energy and junction strength) }\end{array}$ & 12 \\
\hline$q$ (exponent parameter) & 1.4 & $\alpha$ (scaling coefficient for $\Delta \tau_{m}^{S}$ ) & 0.38 \\
\hline $\begin{array}{c}\rho_{\text {cell, }}^{S} \text { (initial dislocation } \\
\text { density in the cell) }\end{array}$ & $4 \times 10^{12} \mathrm{~m}^{-2}$ & $\begin{array}{c}\rho_{W, 0}^{S} \text { (initial dislocation density } \\
\text { in the cell wall) }\end{array}$ & $1 \times 10^{13} \mathrm{~m}^{-2}$ \\
\hline$\chi_{e}$ (entropy factor) & 0.5 & $D_{v}^{0}($ vacancy diffusion constant $)$ & $\begin{array}{l}7.87 \\
\cdot 10^{-7} \mathrm{~m}^{2} \mathrm{~s}^{-1} \\
\end{array}$ \\
\hline$k_{1}$ (material constant) & 0.12 & $E_{m}^{v}$ (vacancy migration energy) & $0.6 \mathrm{eV}$ \\
\hline$k_{2}$ (material constant) & 85 & $E_{f}^{v}$ (vacancy formation energy) & $1.7 \mathrm{eV}$ \\
\hline$k_{3}$ (material constant) & $5.0 \cdot 10^{9}$ & $S_{f}^{v}$ (vacancy formation entropy) & $3.6 \cdot 10^{-4} \mathrm{eV} / \mathrm{K}$ \\
\hline$k_{\mathrm{c}}$ (material constant) & 0.1 & $A_{\text {coble }}($ coble creep parameter) & $1.1 \cdot 10^{-8}$ \\
\hline$g_{0}($ coefficient $)$ & $1.724 \mathrm{eV}$ & $Q_{g b}($ coble creep parameter $)$ & $0.34 \mathrm{eV}$ \\
\hline$g_{1}($ coefficient $)$ & $\begin{array}{r}-1.2 \times 10^{-4} \mathrm{eV} \\
/ \mathrm{K}\end{array}$ & $\begin{array}{c}r_{d} \text { (inner radius of dislocation } \\
\text { control volume) }\end{array}$ & $2 b$ \\
\hline$g_{2}$ (coefficient) & \begin{tabular}{|l|}
$-2.79 \times 10^{-8} \mathrm{eV}$ \\
$/ \mathrm{K}^{2}$
\end{tabular} & $\begin{array}{c}r_{\infty} \text { (outer radius of dislocation } \\
\text { control volume) }\end{array}$ & $200 b$ \\
\hline$g_{3}$ (coefficient) & $\begin{array}{l}-5.93 \\
\times 10^{-11} \mathrm{eV} / \mathrm{K}^{3}\end{array}$ & $\begin{array}{c}l_{\rho} \text { (average distance to climb } \\
\text { for dislocation obstacle) }\end{array}$ & $2 b$ \\
\hline
\end{tabular}


(a)

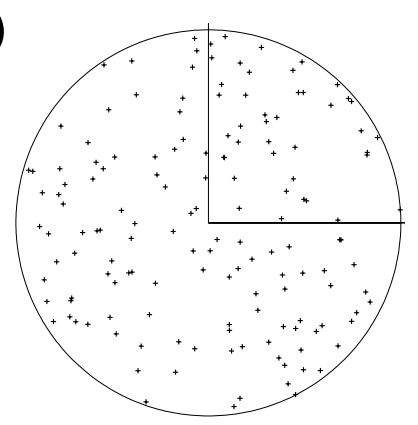

(c)

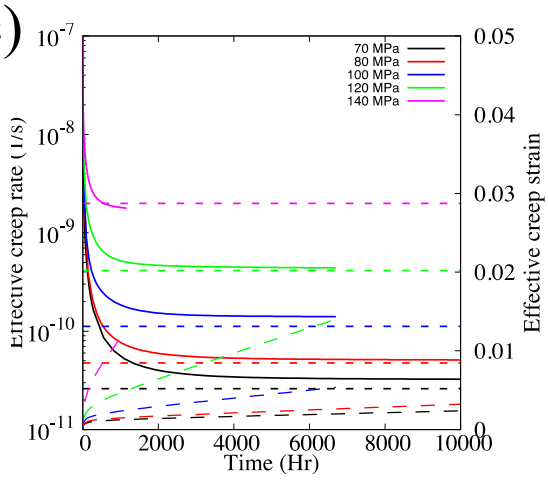

(e)
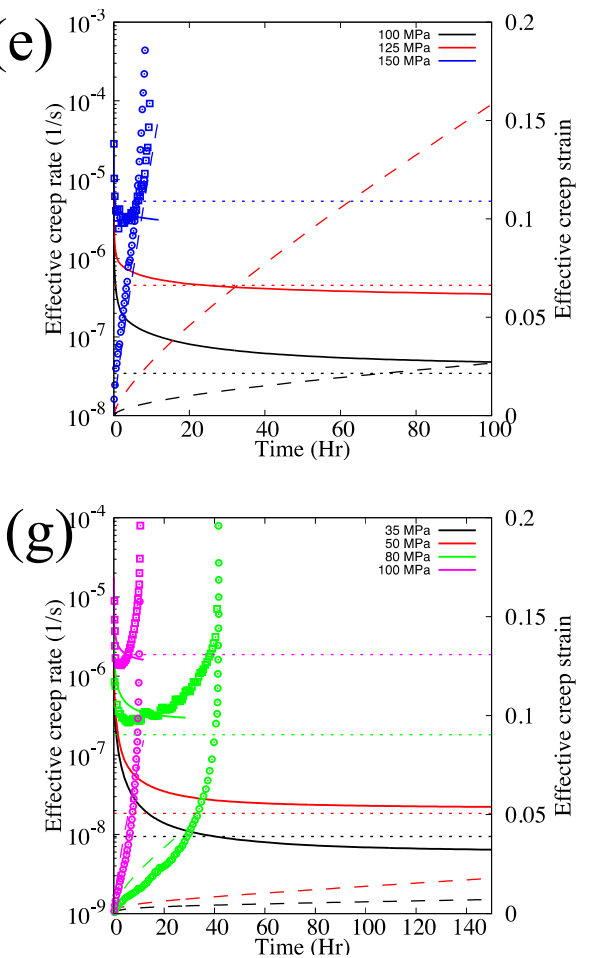

(b)

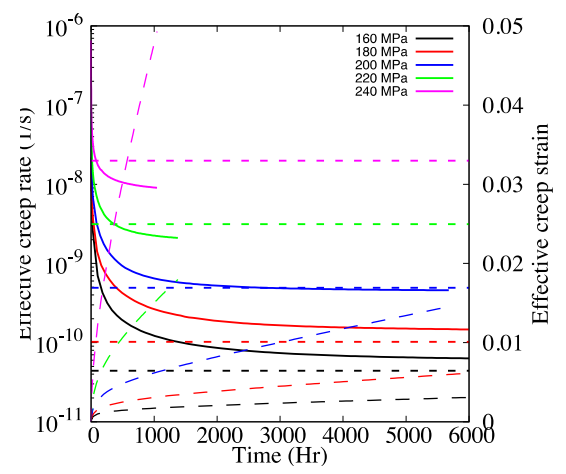

(d)

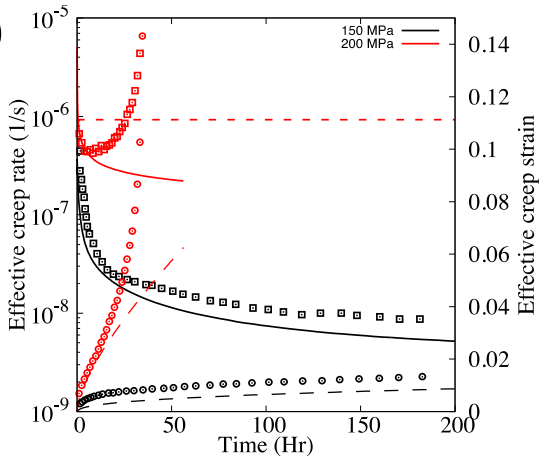

(f)

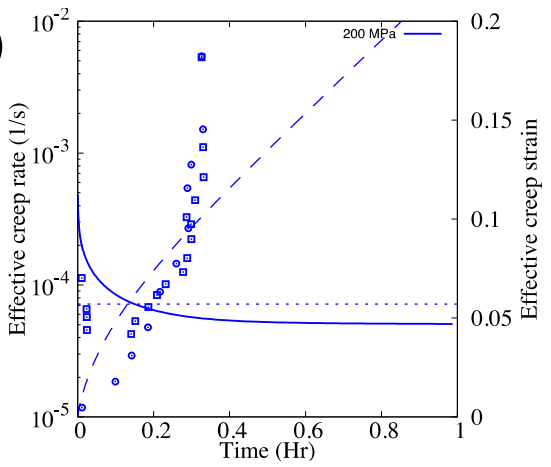

(h)

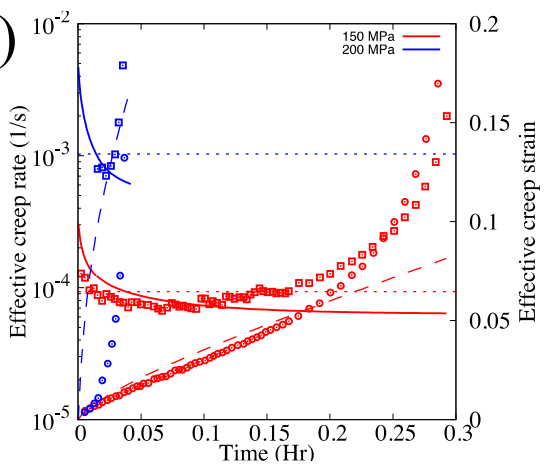

Figure 3.1: (a) Pole figure for the initial random texture of 50 grains. VPSC model predicted thermal creep behavior of P91 alloy as a function of stress at (b) $823 \mathrm{~K}$; (c) and (d) $873 \mathrm{~K}$; (e) and (f) $923 \mathrm{~K}$; (g) and (h) $973 \mathrm{~K}$. The dotted lines represent the experimentally measured steady state creep rate. Similarly, the symbols represent the experimental observations. The solid lines, dotted lines, and square symbols correspond to creep rate plotted on the primary-vertical axis. The dashed lines and circle symbols correspond to creep strain plotted on the secondary vertical axis. 


\subsection{Database Generation}

Polycrystalline responses are gathered in a database to be fit with the SM. The database is made of creep simulations (17,227 in training, 2,859 in testing) to span operating conditions of interest for application with the geometry of the engineering component to which this model is applied later in this report, 1 $150 \mathrm{MPa}$ applied effective stress, and temperatures from 780 to $900 \mathrm{~K}$. To include the effect of a material's pedigree within the SM, parameters are included to account for variations in dislocation density and in MX precipitate content. The database is gathered to capture the relationship between the inputs considered $\left[\bar{\varepsilon}_{v m}, \bar{\rho}_{c e l l}, \bar{\rho}_{W}, T, \bar{\sigma}_{v m}, \phi_{M X}\right]$, and the isotropic rates $\dot{\bar{\varepsilon}}_{v m}, \dot{\bar{\rho}}_{c e l l}$, and $\dot{\bar{\rho}}_{W}$. The SM produces these rates via polynomial regression on the inputs. Notably, the inputs and outputs are transformed to improve the accuracy of the regression. Numerous CP-based creep test simulations are performed for which initial dislocation density and precipitate content are varied. The database is assembled using polycrystal-scale quantities defined using the parameters of the CP-based model. The CP-based model considers both $\{110\}<111>$ and $\{112\}<111>$ slip modes. The effective dislocation density parameters for cells, $\bar{\rho}_{\text {cell }}$, and cell walls, $\bar{\rho}_{W}$, are determined by averaging the slip-system specific densities in each of the 50 grains simulated. The phase fraction of MX precipitates, $\phi_{M X}$, is determined from the number density of MX precipitates, $N_{M X}$, and the diameter of MX precipitates, $d_{M X}$, as

$$
\phi_{M X}=\frac{\pi N_{M X} \pi d_{M X}}{6}
$$

where

$$
d_{M X}=3 \times 10^{-8}\left(1+\frac{\left[\log \phi_{M X}+7.77\right]}{6.692}\right)
$$

To constrain the MX precipitate content to be univariate, an arbitrary relationship is defined that allows $d_{M X}$ to increase with increasing $\phi_{M X}$. Using Equation 3.27 with Equation 3.28, both $N_{M X}$ and $d_{M X}$ can be identified from $\phi_{M X}$. The initial values of $\phi_{M X}, \bar{\rho}_{\text {cell }}$, and $\bar{\rho}_{W}$ were varied to encompass variations in material pedigree. Characterization of P91 alloy has shown values of $d_{M X}$ of around $30 \mathrm{~nm}[1,17-19]$ that can increase to $60 \mathrm{~nm}$ after thermal aging [20]. Dislocation densities in the material may vary as well, and characterization informed the ranges assigned to the values as well [18]. The ranges of microstructure parameters and operating conditions included in the simulation database are defined in Table 3.2. A systematic random sampling method is employed to sample these ranges [21]. The simulations are also constrained by a maximum strain $(8 \%)$ and maximum time $\left(10^{6} \mathrm{hr}\right)$.

Table 3.2: Ranges of the polycrystal-level parameter values gathered in the simulation database

\begin{tabular}{|l|l|}
\hline Variable & Allowable Bounds \\
\hline Cell Dislocation Density $\left(10^{12} \mathrm{~m}^{-2}\right)$ & {$[1,6]$} \\
\hline Wall Dislocation Density $\left(10^{12} \mathrm{~m}^{-2}\right)$ & {$[6,18]$} \\
\hline MX Phase Fraction & {$[0.0005,0.1]$} \\
\hline von Mises Stress $(\mathrm{MPa})$ & {$[1,150]$} \\
\hline Effective Strain $(\mathrm{m} / \mathrm{m})$ & {$[0.0,0.08]$} \\
\hline Temperature $\left({ }^{\circ} \mathrm{C}\right)$ & {$[507,627]$} \\
\hline
\end{tabular}




\subsection{Surrogate Model Formulation}

The SM assumes an isotropic viscoplastic response. Accordingly, a Prandtl-Reuss flow rule is used to determine the tensorial viscoplastic strain rate from an effective viscoplastic strain rate,

$$
\dot{\varepsilon}_{i j}=\dot{\bar{\varepsilon}}_{v m} \frac{3}{2} \frac{S_{i j}}{\bar{\sigma}_{v m}}
$$

where $S_{i j}$ is the deviatoric stress tensor, and $\bar{\sigma}_{v m}=\sqrt{\frac{3}{2} S_{i j} S_{i j}}$ denotes the von Mises stress. The value of $\dot{\bar{\varepsilon}}_{v m}$ and dislocation evolution rates $\dot{\bar{\rho}}_{c e l l}$ and $\dot{\bar{\rho}}_{W}$ are determined using polynomial regression models. To increase the fidelity of the regression models, multiple regression models are used for each output, with individual regression models covering a subregion of the input domain. These models (or 'tiles') are used together to cover the full domain of interest. Within each tile, a Legendre polynomial expansion provides the regression model. $P^{i}(x)$ denotes a polynomial of degree $i$ for input $x$. The range of $x$ is normalized to a $[-1,1]$ interval in defining the polynomial. In addition, transformations of the inputs and output quantities are included in the formulation. The regression functions of the transformed values are thus formulated:

$$
\begin{aligned}
& \dot{\varepsilon}_{v m}^{* *} \sim \sum_{0 \leq i \ldots n \leq N_{\mathrm{deg}}} \alpha_{\varepsilon}^{i j k l m n} P^{i}\left(\bar{\rho}_{c e l l}^{*}\right) P^{j}\left(\bar{\rho}_{w}^{*}\right) P^{k}\left(T^{*}\right) P^{l}\left(\sigma_{v m}^{*}\right) P^{m}\left(\varepsilon_{v m}^{*}\right) P^{n}\left(\phi_{M X}^{*}\right) \\
& \dot{\bar{\rho}}_{c e l l}^{* *} \sim \sum_{0 \leq i \ldots n \leq N_{\mathrm{deg}}} \alpha_{\rho_{\text {cell }}}^{i j k l m n} P^{i}\left(\bar{\rho}_{c e l l}^{*}\right) P^{j}\left(\bar{\rho}_{w}^{*}\right) P^{k}\left(T^{*}\right) P^{l}\left(\sigma_{v m}^{*}\right) P^{m}\left(\varepsilon_{v m}^{*}\right) P^{n}\left(\phi_{M X}^{*}\right) \\
& \dot{\bar{\rho}}_{w}^{* *} \sim \sum_{0 \leq i \ldots n \leq N_{\mathrm{deg}}} \alpha_{\rho_{w}}^{i j k l m n} P^{i}\left(\bar{\rho}_{c e l l}^{*}\right) P^{j}\left(\bar{\rho}_{w}^{*}\right) P^{k}\left(T^{*}\right) P^{l}\left(\sigma_{v m}^{*}\right) P^{m}\left(\varepsilon_{v m}^{*}\right) P^{n}\left(\phi_{M X}^{*}\right)
\end{aligned}
$$

where $\alpha_{\varepsilon}^{i j k l m n}$ are the regression coefficients, specific to each output, and $N_{\text {deg }}$ is the maximum degree of polynomials used in the model. When $N_{\text {deg }}=2$, there are $\left(N_{\text {deg }}+1\right)^{N_{\text {inputs }}}=729$ values in $\alpha_{\varepsilon}^{i j k l m n}$. The symbols $*$ and $* *$ indicate the input or output has been transformed.

The transformations are used because strain rates rarely have linear dependence on $T, \bar{\sigma}_{v m}$, or microstructure. The polynomial regression function is most suitable when fitting linear (or linearized) relations. The transformations are performed prior to the normalization to $[-1,1]$. The transformations used to linearize P91 input-output relations are shown in Table 1.2. A symmetric log transformation is used for the dislocation density evolution rates. This transformation is defined as per Table 3.3, where $\mathrm{y}$ is the transformed output, $\mathrm{z}$ is the original output, and $m$ is a constant used to tune the transformation. Within Table 3.3, this transform is denoted as $p w(z, m)$.

The aforementioned regression models are used to tile across the temperature and stress ranges. Each tile is defined on a temperature subrange (one of [780,836], [804,876], and [844,900] K) and a stress subrange (out of $[1.0,70.5],[30.8,120.2]$, and $[80.5,150] \mathrm{MPa}$ ). Nine tiles in total are presented in Figure 3.2. The overlapping regions are determined using a sigmoid weighting function to blend the results of the overlapped tiles. The sigmoid function is defined as

$$
w_{1}=\frac{1}{1+\exp \left(\frac{4 v}{v^{2}-1}\right)}, w_{2}=1-w_{1}
$$

where the value of $v \in(-1,1)$ is determined in terms of the overlapping input dimension (either $T$ or $\bar{\sigma}_{v m}$ ) as,

$$
v=\frac{2\left(x-x_{\min }\right)}{x_{\max }-x_{\min }}-1
$$


Table 3.3: Transformations of the input and output values used to linearize the regression functions for P91.

\begin{tabular}{|c|c|c|c|c|c|c|c|}
\hline \multicolumn{2}{|c|}{ Inputs } & $\varepsilon_{v m}^{*}$ & $\begin{array}{c}\rho_{\text {cell }}^{*} \\
\left(m^{-2}\right)\end{array}$ & $\begin{array}{c}\rho_{W}^{*} \\
\left(m^{-2}\right)\end{array}$ & $\begin{array}{c}\sigma_{v m}^{*} \\
(\mathrm{MPa})\end{array}$ & $T^{*}(\mathrm{~K})$ & $\phi_{M X}$ \\
\hline$\dot{\varepsilon}_{v m}^{* *}$ & $\ln \left(\dot{\varepsilon}_{v m}\right)$ & $\begin{array}{c}\ln \left(\varepsilon_{v m}\right. \\
\left.+9 \times 10^{-5}\right)\end{array}$ & $\rho_{\text {cell }}$ & $\rho_{W}$ & $\begin{array}{c}\ln \left(\sigma_{v m}\right. \\
+1)\end{array}$ & $T$ & $\begin{array}{c}\ln \left(\phi_{M X}\right. \\
\left.+10^{-3}\right)\end{array}$ \\
\hline$\dot{\bar{\rho}}_{\text {cell }}^{* *}$ & $p w\left(-\frac{\dot{\rho}_{\text {cell }}}{\rho_{\text {cell }}}, 10^{-9}\right)$ & $\begin{array}{c}\ln \left(\varepsilon_{v m}+9 \times\right. \\
\left.10^{-5}\right)\end{array}$ & $\begin{array}{c}\ln \left(\rho_{\text {cell }}+\right. \\
1)\end{array}$ & $\begin{array}{c}\ln \left(\rho_{W}+\right. \\
1)\end{array}$ & $\begin{array}{c}\ln \left(\sigma_{v m}\right. \\
+1)\end{array}$ & $T$ & $\begin{array}{c}\ln \left(\phi_{M X}\right. \\
\left.+10^{-3}\right)\end{array}$ \\
\hline$\dot{\bar{\rho}}_{w}^{* *}$ & $p w\left(-\frac{\dot{\rho}_{W}}{\rho_{W}}, 10^{-9}\right)$ & $\begin{array}{c}\ln \left(\varepsilon_{v m}\right. \\
\left.+9 \times 10^{-5}\right)\end{array}$ & $\begin{array}{c}\ln \left(\rho_{\text {cell }}+\right. \\
1)\end{array}$ & $\begin{array}{c}\ln \left(\rho_{W}\right. \\
+1)\end{array}$ & $\begin{array}{c}\ln \left(\sigma_{v m}\right. \\
+1)\end{array}$ & $T$ & $\begin{array}{c}\ln \left(\phi_{M X}\right. \\
\left.+10^{-3}\right)\end{array}$ \\
\hline
\end{tabular}

where $x$ denotes the overlapped input. Where four tiles are overlapping, the sigmoid weighting is performed to merge along both $T$ and $\bar{\sigma}_{v m}$, one dimension at at time. 


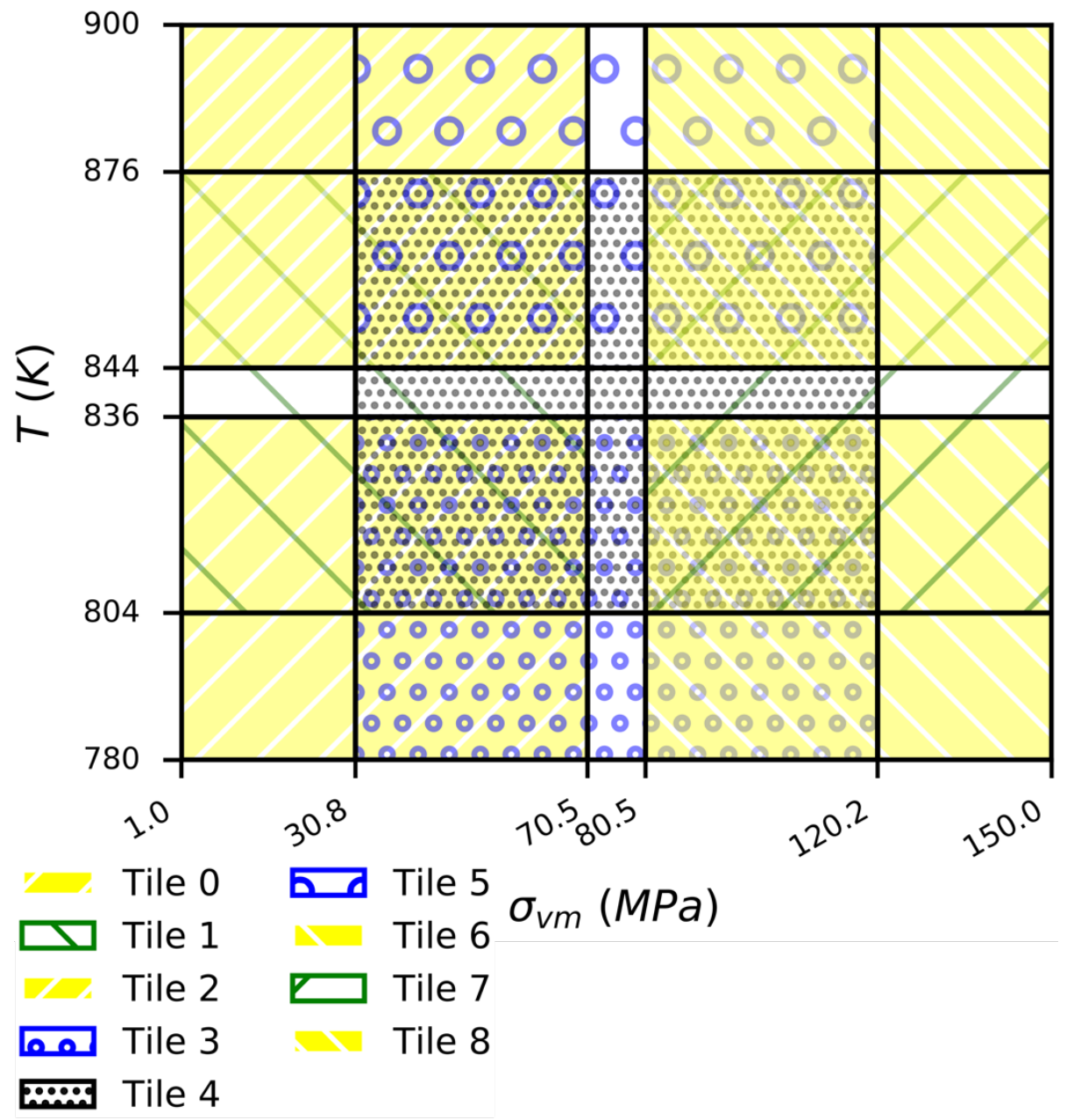

Figure 3.2: Layout of the overlapping multiple regression model tiles. 

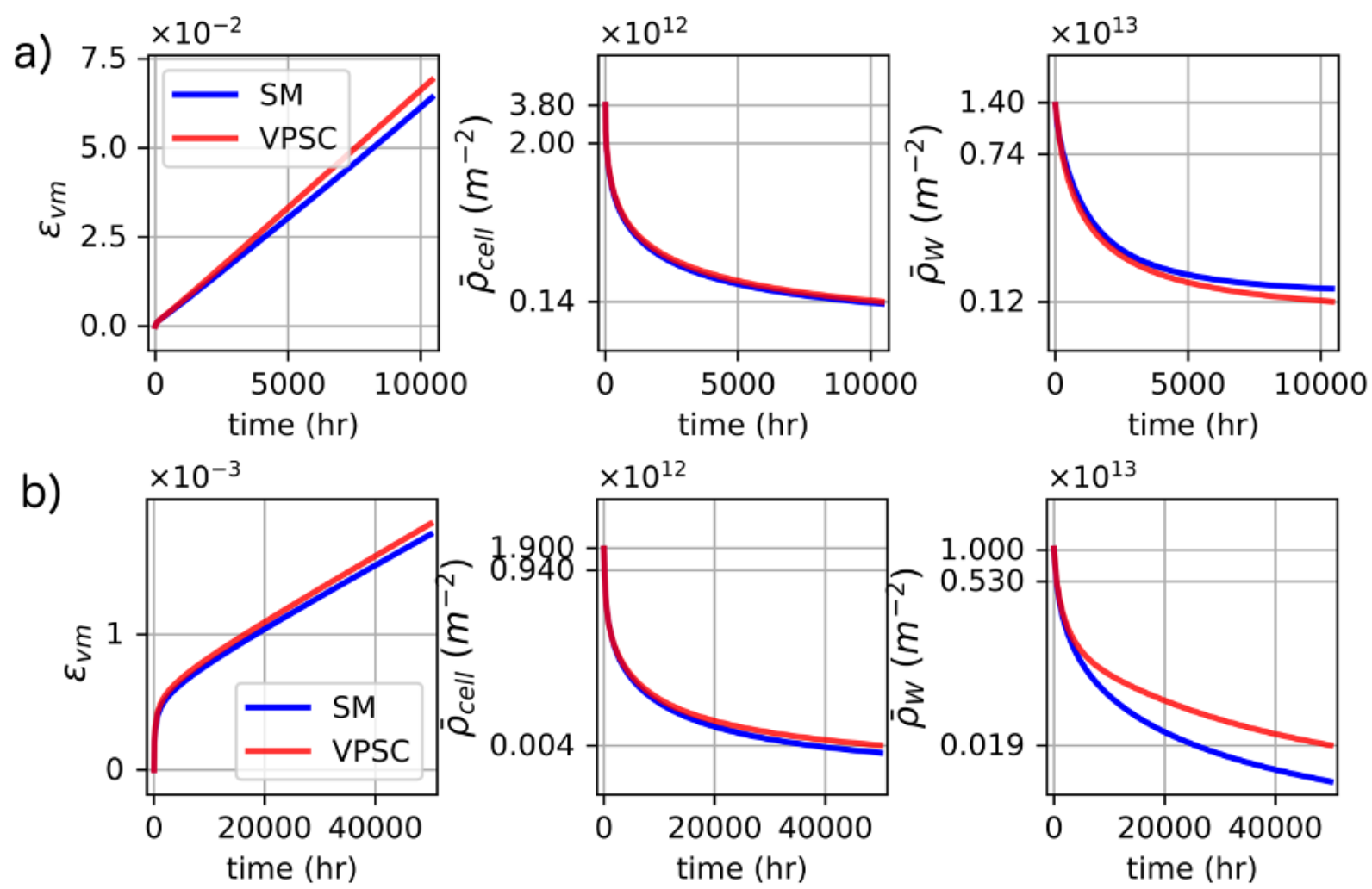

Figure 3.3: A comparison of the SM predictions with VPSC for two validation creep test simulations. From left to right, the evolution of $\bar{\varepsilon}_{v m}, \bar{\rho}_{c e l l}$, and $\bar{\rho}_{W}$ are shown, with $\bar{\rho}_{c e l l}$ and $\bar{\rho}_{W}$ values given on a $\log$ scale. Inputs for case (a) are $\left[\bar{\rho}_{\text {cell }}=3.8 \times 10^{12} \mathrm{~m}^{-2}, \bar{\rho}_{W}=1.36 \times 10^{13} \mathrm{~m}^{-2}, \mathrm{~T}=869 \mathrm{~K}, \bar{\sigma}_{v m}=\right.$ $\left.134 \mathrm{MPa}, \phi_{M X}=0.083\right]$, and for case (b) $\left[\bar{\rho}_{\text {cell }}=1.88 \times 10^{12} \mathrm{~m}^{-2}, \bar{\rho}_{W}=1.03 \times 10^{13} \mathrm{~m}^{-2}, \mathrm{~T}=886\right.$ $\left.\mathrm{K}, \bar{\sigma}_{v m}=49 \mathrm{MPa}, \phi_{M X}=0.0076\right]$ 


\subsection{Surrogate Model Validation}

The validation of the SM is performed in two parts, beginning with the SM replication of CP creep test simulations spanning the input domain. Following analysis of these results, the SM is applied to complex loading conditions, constant stress with a gradual increase in temperature, a jump in applied stress, a ramping up of applied stress, and a cyclic stress condition. These complex loadings are never used in training the SM. The predictions of the SM in these cases are given here to interrogate the capabilities and limitations of the SM. These cases are also used to discuss the fidelity of the SM when used in FEM simulations.

\subsubsection{Creep Test Validation}

Validation of the SM is performed using 300 CP-based creep test simulations reserved separate from the training and testing data. The SM predicts the entire creep test simulation from the values of the inputs at $t=0$ and the magnitudes of the time-steps. These validation cases test the accuracy of the SM over interpolated regions of the targeted operating conditions. Further, the simulations present a chance for errors in one time-step to propagate to subsequent time-steps. The validation tests are critical to identifying such errors. Two validation cases are shown in Figure 3.3. The cases show the SM has a high fidelity to the VPSC results. There is a large difference in the magnitude of the primary creep between the two cases. The SM accurately captures this difference. The steady-state creep rate is increasingly important at longer lifetimes, and in both cases the SM matches this steady-state rate well. The full validation of the SM involves 300 simulations similar to the simulations in Figure 3.3. To summarize the results, the mean squared relative error (MSRE) is used. For the predicted strains in Figure 3.3 (a) and (b), the MSRE values are 0.0044 and 0.0146, respectively. This value is presented for all 300 cases in Figure 3.4. In Figure 3.4 (a) and (b), each creep test simulation is plotted as a point with respect to the $T$ and $\bar{\sigma}_{v m}$ imposed for that test. In (a) these points indicate $M S R E\left(\bar{\varepsilon}_{v m}\right)$ by color, and in (b) the points indicate the ratio of the predicted creep rate at steady state to that calculated with VPSC. The plots show excellent fidelity, excluding the triangular region of high $T$ and high $\bar{\sigma}_{v m}$, and excluding the extreme low $\bar{\sigma}_{v m}$ at high $T$.

\subsubsection{Complex Loading Conditions}

The SM is trained on only creep test simulations, however it is desired that the SM can be applied under more complex conditions. In FEM, boundary conditions are subject to change over the course of the simulation. To support the use of the SM in FEM, the SM is validated against VPSC simulations in which either temperature or applied stress are allowed to vary with time. Four such complex loadings are defined for this purpose: a stress jump condition, a stress ramp condition, a temperature ramp condition, and a stress cycle condition.

The validation of the SM in a stress-jump condition is shown in Figure 3.5. The stress jump refers to an instantaneous doubling of the applied stress from 33.5 MPa to $67 \mathrm{MPa}$ after $70 \mathrm{hrs}$ of an otherwise monotonic creep test simulation. The applied stress is shown as a function of time in Figure 3.5. The SM replicates the VPSC simulation results with an excellent level of fidelity. The SM performance in a stress-ramp simulation is shown in Figure 3.6. The stress-ramp refers to a gradual increase in applied stress after an initial constant stress period of $28 \mathrm{hrs}$. The stress is increased from $30.9 \mathrm{MPa}$ to $61.8 \mathrm{MPa}$ at a rate of $3.09 \times 10^{-3} \mathrm{MPa} / \mathrm{s}$. The SM demonstrates an excellent fidelity in the stress ramp case. These results support the use of the SM when applied stress is subject to changes, whether rapid or gradual.

A simulation in which the temperature is rapidly increased $(0.5 \mathrm{~K} / \mathrm{s})$ from an initial value of $790 \mathrm{~K}$ to $890 \mathrm{~K}$ is shown in Figure 3.7, referred to as a temperature ramp. The SM and the VPSC simulations show

initial agreement. As the temperature is increased, the expected increases in rates $\dot{\bar{\varepsilon}}_{v m}, \dot{\bar{\rho}}_{c e l l}$, and $\dot{\bar{\rho}}_{W}$ are 

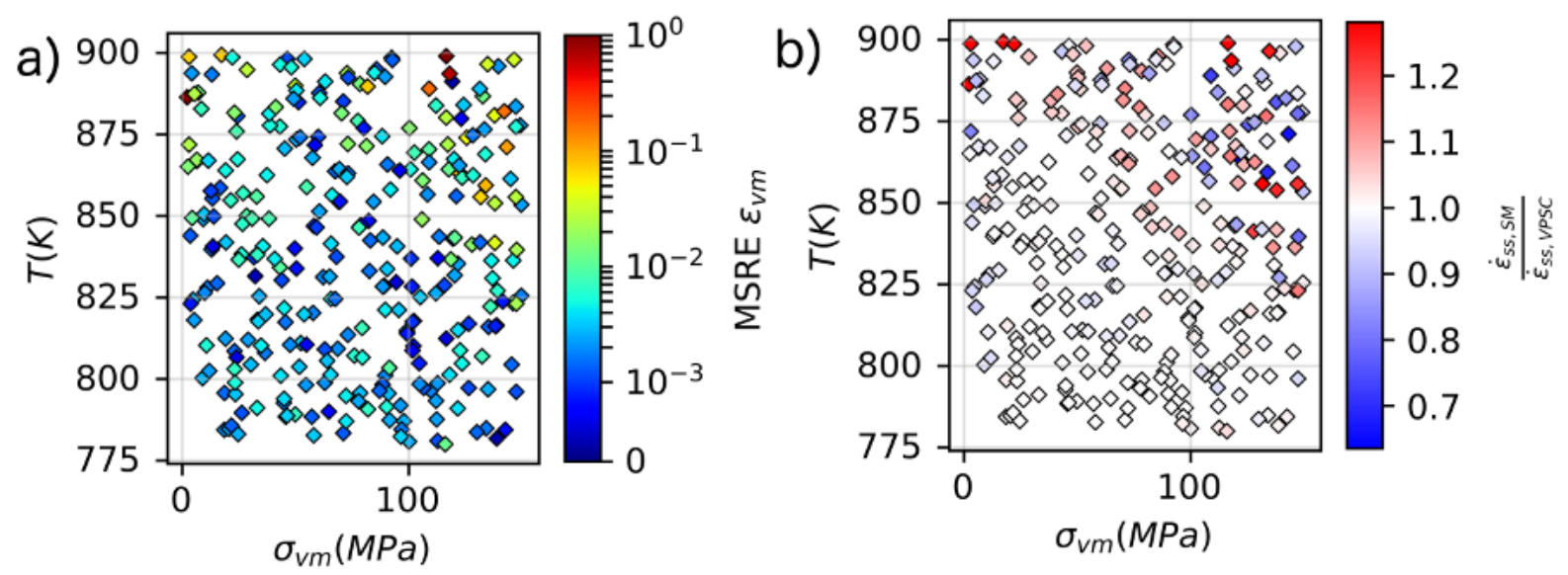

Figure 3.4: The (a) MSRE and (b) ratio of creep rate at steady-state for SM and VPSC simulations, plotted by the imposed and conditions for each of 300 validation creep test simulations.
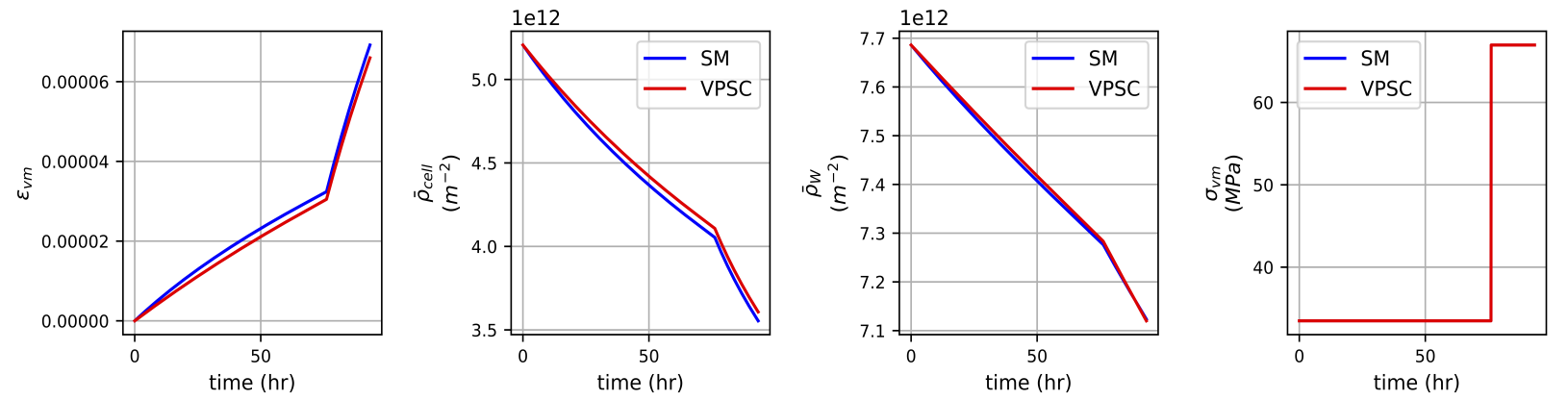

Figure 3.5: A validation of the SM in a stress-jump loading condition. The SM is shown in blue and the VPSC result is shown in red. The initial input values are $\left[\bar{\rho}_{\text {cell }}=5.2 \times 10^{12} \mathrm{~m}^{-2}, \bar{\rho}_{W}=7.7 \times 10^{12} \mathrm{~m}^{-2}\right.$, $\left.\mathrm{T}=867 \mathrm{~K}, \bar{\sigma}_{v m}=33.5 \mathrm{MPa}, \phi_{M X}=0.0596\right]$ The stress instantaneously doubles after a period of 70 hrs.
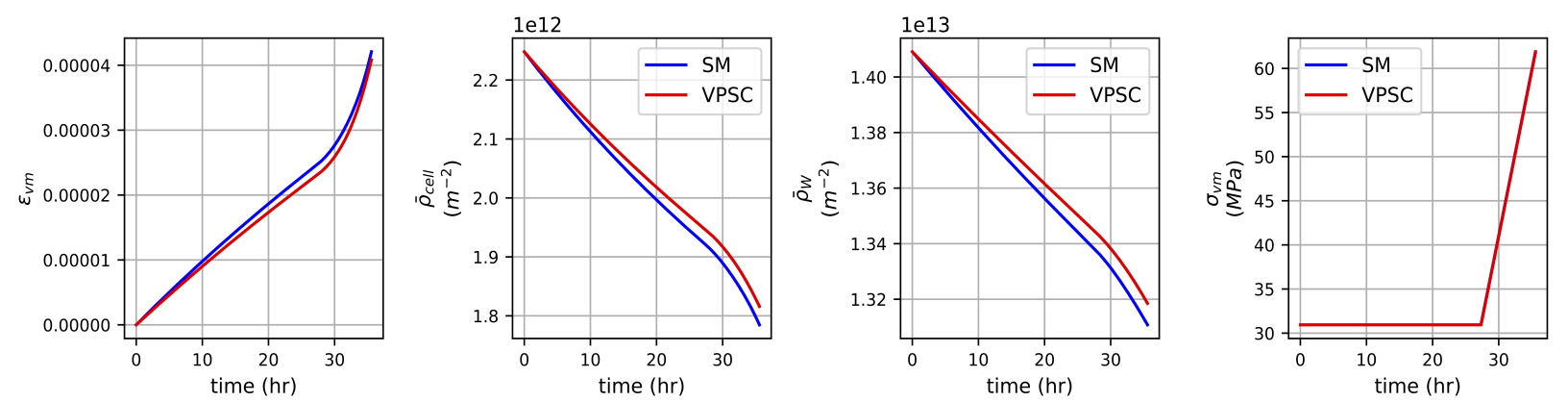

Figure 3.6: A validation of the SM in a stress-jump loading condition. The SM is shown in blue and the VPSC result is shown in red. The initial input values are $\left[\bar{\rho}_{\text {cell }}=2.3 \times 10^{12} \mathrm{~m}^{-2}, \bar{\rho}_{W}=1.41 \times 10^{13} \mathrm{~m}^{-2}\right.$, $\mathrm{T}=889 \mathrm{~K}, \bar{\sigma}_{v m}=30.9 \mathrm{MPa}, \phi_{M X}=0.0513$ ] The stress doubles gradually after a period of $28 \mathrm{hrs}$ at a rate of $3.09 \times 10^{-3} \mathrm{MPa} \cdot \mathrm{s}^{-1}$. 

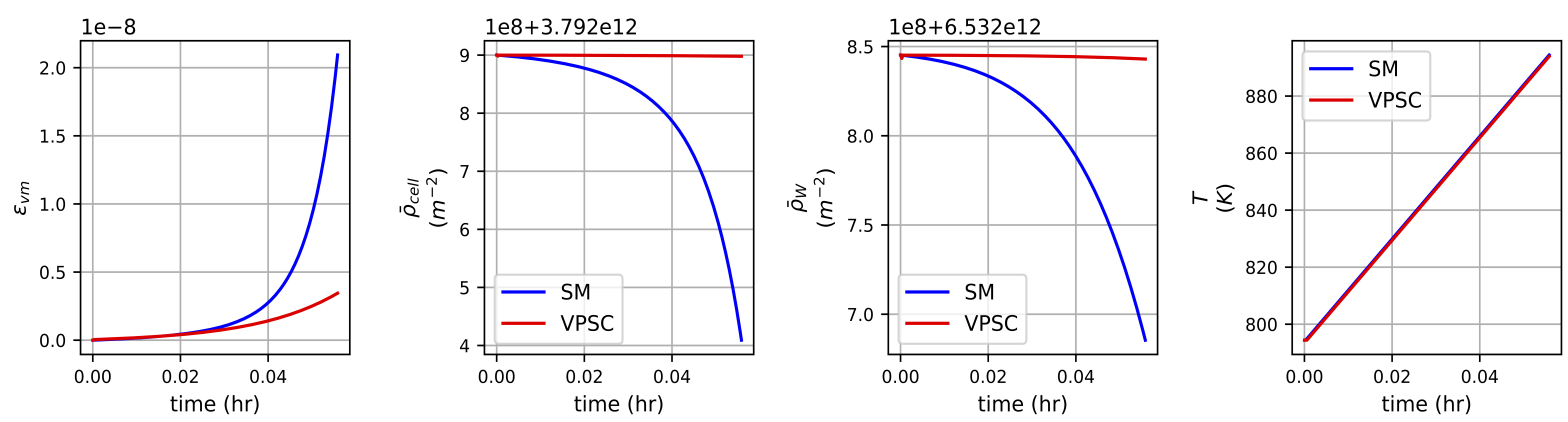

Figure 3.7: A validation of the SM in a temperature ramp loading condition. The SM is shown in blue and the VPSC result is shown in red. The initial input values are $\left[\bar{\rho}_{\text {cell }}=1.77 \times 10^{12} \mathrm{~m}^{-2}, \bar{\rho}_{W}=\right.$ $\left.1.35 \times 10^{13} \mathrm{~m}^{-2}, \mathrm{~T}=790 \mathrm{~K}, \bar{\sigma}_{v m}=38.7 \mathrm{MPa}, \phi_{M X}=0.0925\right]$
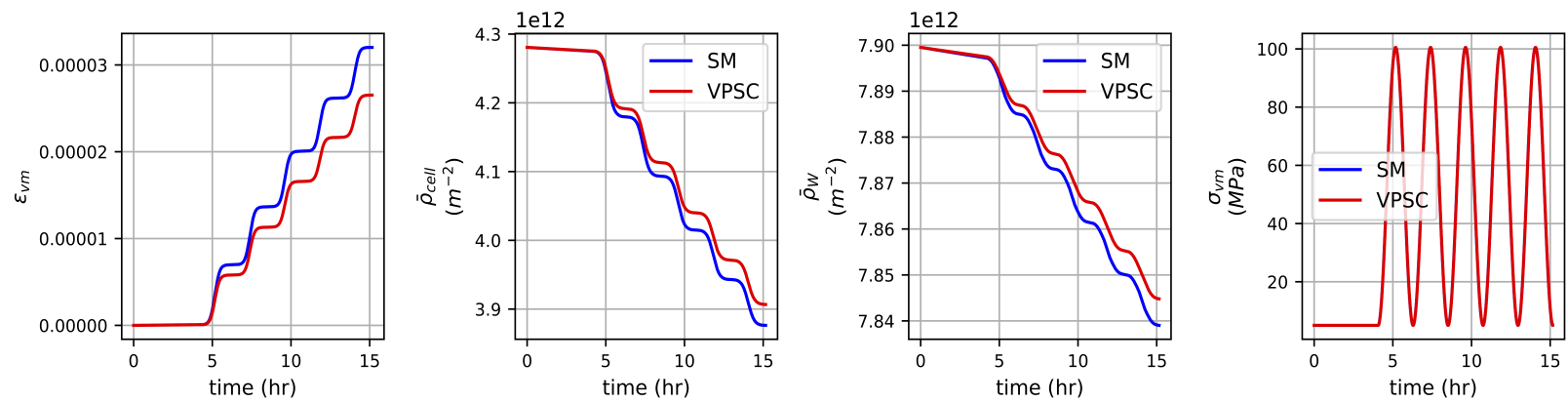

Figure 3.8: A validation of the SM in a stress-cycling loading condition. The SM is shown in blue and the VPSC result is shown in red. The initial input values are. After $4 \mathrm{hrs,} \mathrm{a} \mathrm{sinusoidal} \mathrm{stress} \mathrm{cycle}$ is imposed, with a maximum stress of and a period of $100 \mathrm{~m}$, for 5 cycles.

produced by both VPSC and SM. The rate increase with temperature is overpredicted by the SM in this case. The fidelity of the SM to VPSC in this simulation is notably lower than in previous cases. Multiple factors distinguish this simulation case from those previously shown. In addition to the introduction of a temperature change, the total duration of the simulation is very short, much shorter than $1 \mathrm{hr}$. The fidelity of the SM under temperature changes is an area of ongoing development.

The suitability of the SM for cyclic stress applications is investigated. A loading is designed in which an initially low stress $(5.0 \mathrm{MPa})$ is applied for $4 \mathrm{hrs}$. Following this, a sinusoidal stress cycle with maximum stress of $100 \mathrm{MPa}$ and period of $100 \mathrm{~m}$ is imposed 5 times. The SM shows good agreement with the VPSC results. 


\section{Bibliography}

[1] P.E. O’Donoghue C.O Murch, S.B. Leen and R.A. Barrett. A physically-based creep damage model for effects of different precipitate types. Mater Sci. Eng. A, 682:714-722, 2017.

[2] W. Wen, L. Capolungo, A. Patra, and C. N. Tomé. A. physics-based crystallographic modeling framework for describing the thermal creep behavior of fe-cr alloys,. Metall Mater. Trans. A., 48, 2017.

[3] W. Wen, A. Kohnert, M. Arul Kumar, L. Capolungo, and C. N. Tomé. Mechanism-based modeling of thermal and irradiation creep behavior: An application to ferritic/martensitic ht9 steel,. Int J. Plast., 126(10263):3, 2020.

[4] R. A. Lebensohn and C. N. Tomé. A self-consistent anisotropic approach for the simulation of plastic deformation and texture development of polycrystals: Application to zirconium alloys,. Acta Metall Mater., 41:2611-2624, 1993.

[5] R. A. Lebensohn, C. N. Tomé, and P. P. Casta Neda. Self-consistent modelling of the mechanical behaviour of viscoplastic polycrystals incorporating intragranular field fluctuations,. Philos Mag., 87:4287-4322, 2007.

[6] H. Wang, L. Capolungo, B. Clausen, and C. N. Tomé. Int a crystal plasticity model based on transition state theory,. J. Plast., 93:251-268, 2017.

[7] P. Franciosi and A. Zaoui. Multislip tests on copper crystals: A junctions hardening effect. Acta Metall., 30:2141-2151, 1982.

[8] E. Arzt and D. S. Wilkinson. Threshold stresses for dislocation climb over hard particles: The effect of an attractive interaction. Acta Metall., 34:1893-1898, 1986.

[9] E. Arzt and J. R"osler. The kinetics of dislocation climb over hard particles-ii. Effects of an attractive particle-dislocation interaction, Acta Metall., 36:1053-1060, 1988.

[10] K. Kitayama, C. N. Tomé, E. F. Rauch, J. J. Gracio, and F. Barlat. A crystallographic dislocation model for describing hardening of polycrystals during strain path changes application to low carbon steels. Int J. Plast, 46:54-69, 2013.

[11] J.-F. Wen, S.-T. Tu, F.-Z. Xuan, X.-W. Zhang, and X.-L. Gao. Effects of stress level and stress state on creep ductility: evaluation of different models,. J Mater. Sci Tech, 32:695-704, 2016.

[12] Y. Estrin. Dislocation theory based constitutive modelling: foundations and applications,. J Mater. ProcessTechnol., 80:33-39, 1998.

[13] M. Basirat, T. Shrestha, G. P. Potirniche, I. Charit, and K. Rink. A study of the creep behavior of modified 9cr-1mo steel using continuum-damage modeling,. Int J. Plast., 37:95-107, 2012.

[14] T. Shrestha, M. Basirat, I. Charit, G. P. Potirniche, K. K. Rink, and U. Sahaym. Creep deformation mechanisms in modified 9cr-1mo steel,. J Nucl. Mater, 423:110-119, 2012. 
[15] K. Kimura, M. Tabuchi, Y. Takahashi, K. Yoshida, and Koichi Yagi. Long-term creep strength and strength reduction factor for welded joints of asme grades 91, 92 and 122 type steels, int. J. Microstruct. Mater Prop., 6:72-90, 2011.

[16] K. Kimura, K. Sawada, H. Kushima, and Y. Toda. Microstructural stability and long term creep strength of grade 91 steel. Energy Mater, 4:176-183, 2009.

[17] G. Golański, J. Jasak, A. Zieliński, C. Kolan, M. Urzynicok, and P. Wieczorek. Quantitative analysis of stability of 9\% cr steel microstructure after long-term ageing,. Arch Metall. Mater, 62, 2017.

[18] C. G. Panait, W. Bendick, A. Fuchsmann, A.-F. Gourgues-Lorenzon, and J. Besson. Study of the microstructure of the grade 91 steel after more than $100,000 \mathrm{~h}$ of creep exposure at $600{ }^{\circ} \mathrm{c}$, . Int $J$. Press Vessels Pip, 87:326-335, 2010.

[19] K. Maruyama, K. Sawada, and J. Koike. Strengthening mechanisms of creep resistant tempered martensitic steel. ISIJ Int, 41:641-653, 2001.

[20] M. Li, K. Natesan, and W. Chen. Report on understanding and predicting effects of thermal aging on microstructure and tensile properties of grade 91 steel for structural components, argonne national lab. (anl), argonne, il (united states). 2017.

[21] J. L. Deutsch and C. V. Deutsch. Latin hypercube sampling with multidimensional uniformity,. J Stat, 142:763-772, 2012. 


\section{Uncertainty Quantification of Engineering-Scale Component Life Predictions}

In this chapter we present the problems used to evaluate the high temperature creep behavior of the Grade 91 alloy material models, described in the previous sections, and the response of the models to material uncertainties. Prior work [1] evaluated the response of the NEML and LAROMANCE Grade 91 material models for a single set of input properties as applied to a 2D and full 3D model. In this work we focus on the statistical high temperature response by sampling the material inputs over the full range of valid parameters for these models. To sample over a large parameter space for multiple parameters, we limited our analysis to $2 \mathrm{D}$ axisymmetric models. Both the 2D axisymmetric spherical nozzle and the pipe cross section shown in the inset are shown in Figure 4.1 and are modeled with the NEML and LAROMANCE Grade 91 materials. The simplified pipe cross section model is fast-running and allows us to evaluate material models over a range of stress states found for a thick walled pressure vessel. The larger sphere nozzle geometry model tests the material models under a more complicated stress state and is a refined 2D version of the 3D coarse sphere nozzle mesh used in [1]. Each geometry is simulated under a constant pressure and temperature for either twenty years or until the maximum principal strain reaches a critical level, whichever occurs first.

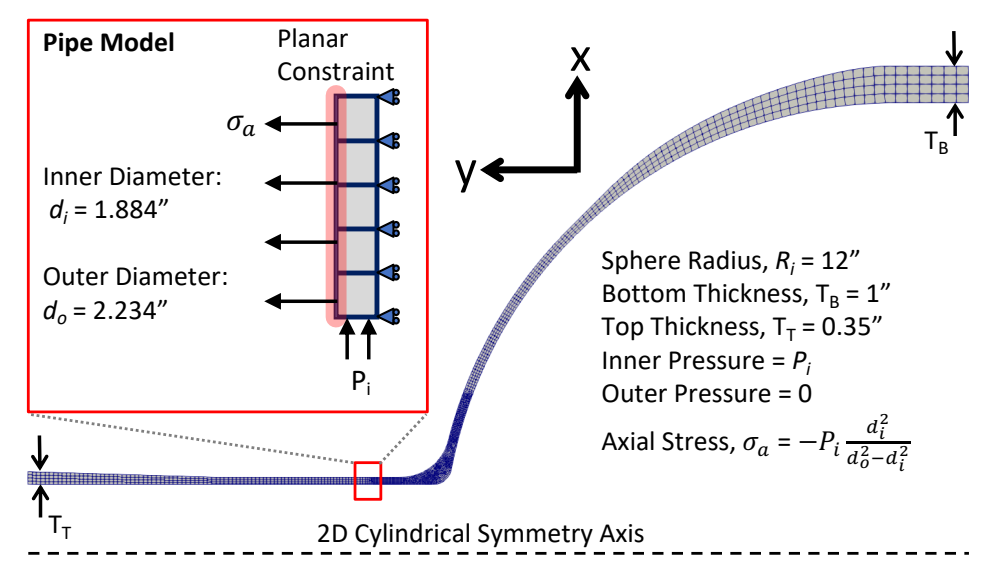

Figure 4.1: Internally pressurized axisymmetric sphere-nozzle problem, with simplified pipe model shown in inset.

The dimensions for the simplified 2D pipe cross section model are taken from the sphere nozzle geometry at the location indicated by the red box in Figure 4.1. Dimensions and boundary conditions are given in Figure 4.1. The pipe cross section is modeled with five 2D axisymmetric QUAD8 elements through the thickness. The boundary conditions applied to the model are RZ-symmetry (i.e., zero x-displacement along the $y$-axis and zero $y$-displacement along the $\mathrm{x}$-axis). The pipe cross section model is pressurized with an internal pressure, $\mathrm{P}$, zero external pressure, and axial stress, $\sigma_{a}$, given by the equation for a long thick walled pressure vessel. To approximate an infinitely long pipe, the nodes on the positive y-surface are constrained to remain planar while the nodes on the bottom surface are constrained to only move along the $\mathrm{x}$-axis. The simulations were initialized with a constant internal pressure ranging from 5-8 MPa and a uniform temperature 
of either $550{ }^{\circ} \mathrm{C}$ or $600{ }^{\circ} \mathrm{C}$.

The maximum von Mises stress in an internally pressurized thick walled cylinder is largest near the internal surface and will be the location of the maximum creep strain. The maximum principal strain we use as the critical measure in the simulation response is derived from the total strain which will include elastic and creep components; however, for the pressure ranges we use in our analysis, the elastic component of strain should be negligible when compared to the creep strain when the overall principal strain reaches $1 \%$.

The sphere nozzle problem shown in Figure 4.1 is also modeled with 2D axisymmetric QUAD8 elements. The model consists of 3,282 elements and 11,044 nodes. Most of the model is meshed with 4 elements through the thickness. The mesh density is then doubled near the nozzle pipe junction where it reaches a maximum of 13 elements through the thickness. The same boundary conditions are applied here as were used for the simplified pipe cross section model (i.e., RZ-symmetry, zero displacement at the negative yboundary, axial stress at the positive y-boundary with nodes constrained to remain planar, varying internal pressure on the negative $\mathrm{x}$-surface and zero pressure along the outer positive $\mathrm{x}$-surface). The dimensions given in Figure 4.1 and used in the 2D axisymmetric model are the same as those used for the 3D sphere nozzle mesh presented in [1]. The simulations were initialized with a constant internal pressure ranging from 1.5-3 MPa and a uniform temperature of either $550{ }^{\circ} \mathrm{C}$ or $600{ }^{\circ} \mathrm{C}$.

The sphere nozzle provides a more complex stress-strain response as opposed to the nearly analytical response of the simplified pipe cross section model. The main complexities come from the stress concentration created by the transition of the nozzle into the pipe wall and from the nonuniform deformation. Due to these two factors the maximum principal strain is not guaranteed to be located at the inner surface and the direction of the maximum principal strain will change spatially.

\subsection{NEML Material Model Results}

In this work, we consider the statistical nature of the models response due to material uncertainties. In our prior work [1] we considered the strain and pressure driven response of the NEML and LAROMANCE P91 models for only a single set of input material parameters. As was done in our prior work, we only consider monotonic loading and therefore do not use any of the NEML material properties fit to cyclic loading experimental data presented in the previous section. For the NEML P91 material model, the MCMC posterior distributions shown in Figure 2.2 with mean values given in Table 2.2 were previously fit to Beta distributions.

We use the MOOSE Stochastic Tools Monte Carlo sampler to efficiently run the simulations and manage the sampling of the material properties for this work. The Beta distribution is currently not supported by the MOOSE Stochastic Tools Monte Carlo sampler; therefore, we refit the posterior distributions for the material properties to truncated normal distributions shown by the magenta lines in Figure 4.2. Values for the truncated normal distributions are given in Table 4.2. We also do not consider damage in these high temperature creep simulations under low stress conditions and therefore do not include the damage model given by Equation 2.5, reducing the number of parameters we fit in Figure 4.2 to five. The NEML P91 model also uses the temperature dependent elastic constants given in Table 4.1. All of our simulations assume homogeneous material properties at the sampled values for a given random realization.

Table 4.1: Temperature dependent elastic constants used for NEML and LAROMANCE P91 model

\begin{tabular}{|c|c|c|c|}
\hline \multicolumn{2}{|c|}{$550^{\circ} \mathrm{C}$} & \multicolumn{2}{|c|}{$600^{\circ} \mathrm{C}$} \\
\hline Young's Modulu & $74 \mathrm{GPa}$ & Young's Modulu & $68 \mathrm{GPa}$ \\
\hline Poisson's Ratio & 0.31 & Poisson's ratio & 0.31 \\
\hline
\end{tabular}


Table 4.2: Posterior MCMC distribution of the NEML model parameters.

\begin{tabular}{cccccc}
\hline \multirow{2}{*}{ parameter } & \multicolumn{2}{c}{$550^{\circ} \mathrm{C}$} & & \multicolumn{2}{c}{$600^{\circ} \mathrm{C}$} \\
\cline { 2 - 3 } \cline { 5 - 6 } & $(\mu, \sigma)$ & Bounds & & $(\mu, \sigma)$ & Bounds \\
\hline$n$ & $(11.09,0.37)$ & {$[10,12]$} & & $(8.455,0.47)$ & {$[6.9,10]$} \\
$\eta$ & $(832.4,23)$ & {$[760,909]$} & & $(750.6,41.9)$ & {$[613,884]$} \\
$\sigma_{0}$ & $(3.769,0.83)$ & {$[1.2,6.5]$} & & $(3.547,0.66)$ & {$[2.0,5.8]$} \\
$Q$ & $(106.9,8.7)$ & {$[78,135]$} & & $(112.6,8.19)$ & {$[87,140]$} \\
$b$ & $(47.99,7.7)$ & {$[30,70]$} & & $(44.00,8.28)$ & {$[21,71]$} \\
\hline
\end{tabular}

Input Parameters from Posterior MCMC ( $\mathrm{T}=550 \mathrm{C})$
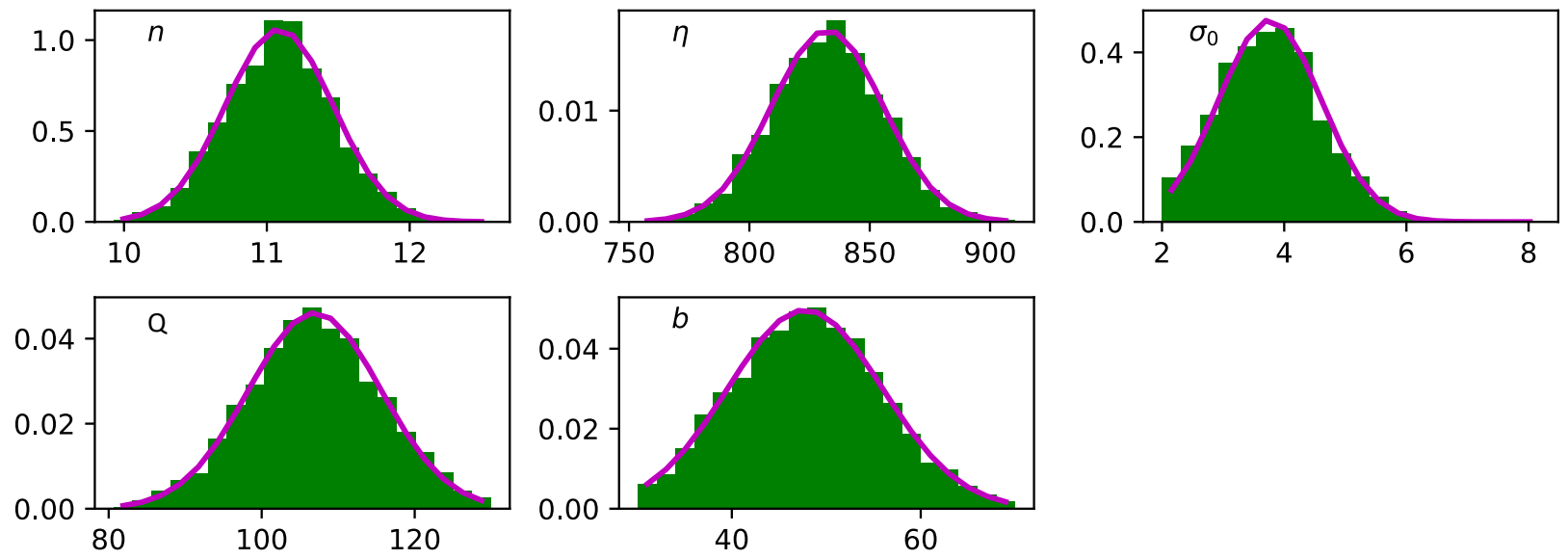

a)

Input Parameters from Posterior MCMC ( $\mathrm{T}=600 \mathrm{C})$
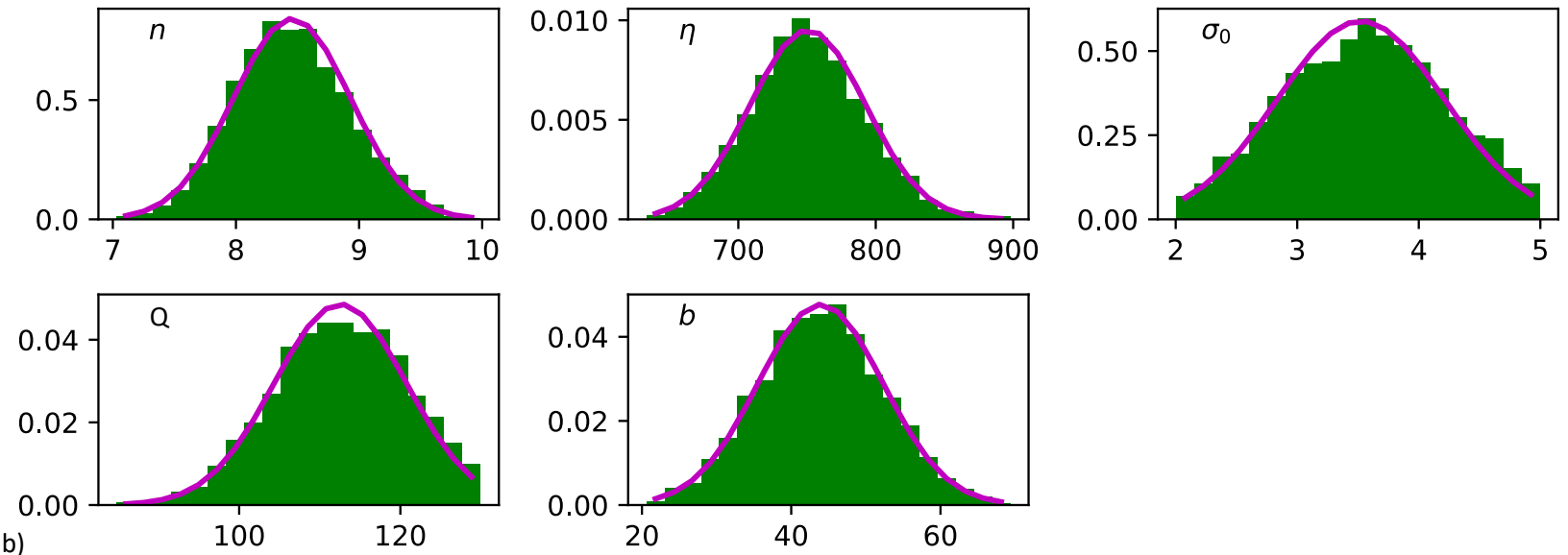

Figure 4.2: Input material parameters for the NEML model. MCMC posterior material property distributions in green fit to truncated normal distributions (magenta) for (a) $550{ }^{\circ} \mathrm{C}$ and (b) $600{ }^{\circ} \mathrm{C}$. Truncated normal distribution properties given in Table 4.2. 


\subsubsection{NEML Simplified Pipe Model Results}

The statistical results for the pressurized pipe cross section model from the inset of Figure 4.1, using the NEML material model are given in this section below. The maximum von Mises stress in an internally pressurized cylinder is highest near the internal surface and will be the location of the maximum creep strain. The maximum principal strain as a function of time is shown in Figure 4.3a for 2000 Monte Carlo samples from the distributions shown in Figure 4.2 and Table 4.2. We see that as the pressure increases, the spread in maximum principal strain increases. As the temperature increases to $600{ }^{\circ} \mathrm{C}$ in Figure $4.2 \mathrm{~b}$, we switch between two different fitted distributions, and the distributions for $\mathrm{T}=600{ }^{\circ} \mathrm{C}$ lead to a larger range of maximum strains. The lines in the plot are semi-transparent, so the more darkly filled regions indicate a higher density of samples following a strain path through that region. Using the line darkness as a guide, we see that although the strain distributions and their slope are increasing for $\mathrm{T}=550{ }^{\circ} \mathrm{C}$, most of the samples still follow a flat line. This observation does not hold for $\mathrm{T}=600{ }^{\circ} \mathrm{C}$ (Figure $4.2 \mathrm{~b}$ ), where the principal strain histories for most samples deviate considerably from a flat line.
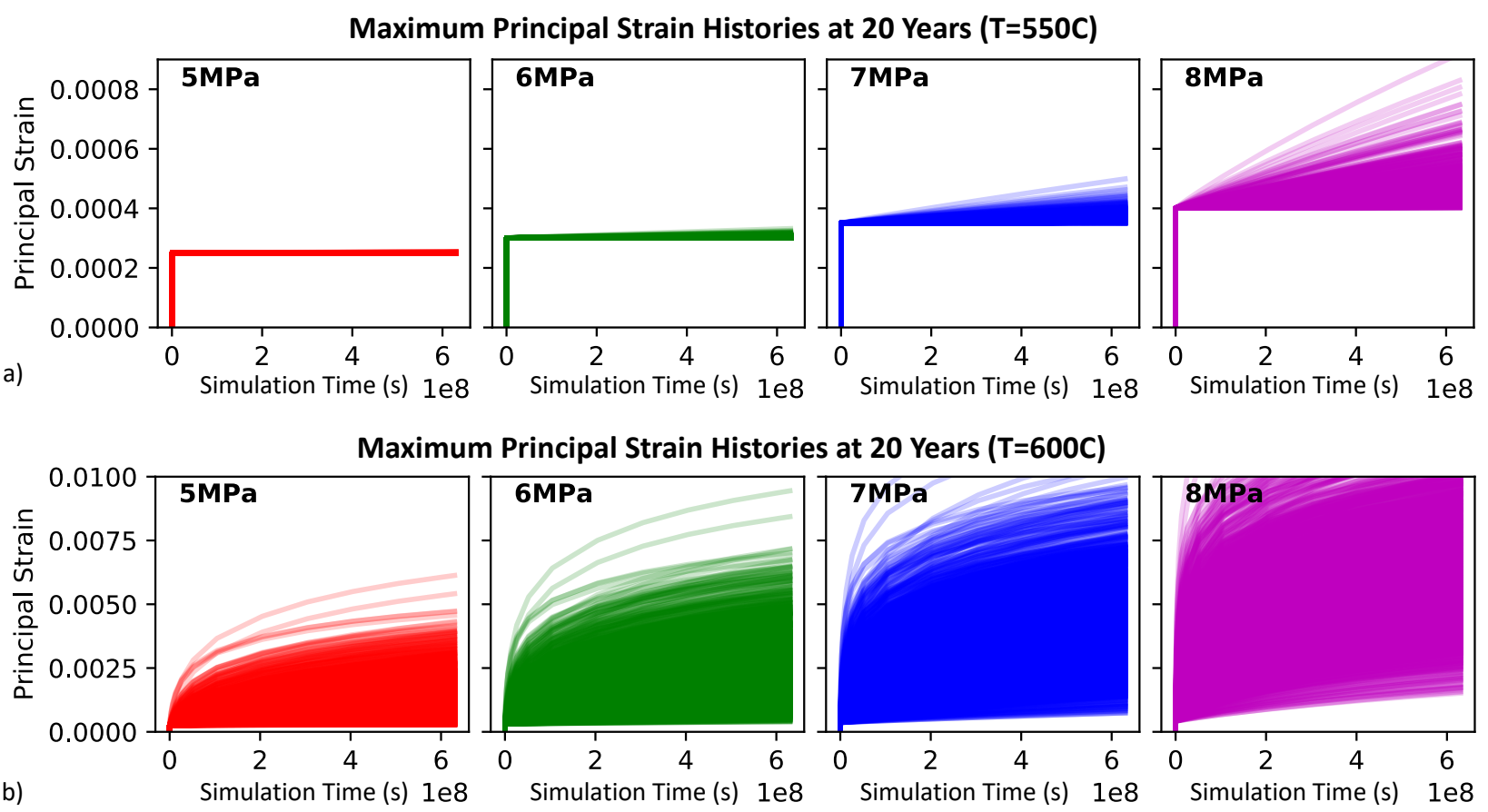

Figure 4.3: Maximum principal strain as a function of time for up to 20 years or until $1 \%$ strain was reached for 2000 Monte Carlo samples of posterior truncated normal distributions shown in Figure 4.2.

The final states of the maximum principal strain at the end of 20 years for the time series of simulations given in Figure 4.3 are shown in Figure 4.4 for the same range of pressures and temperatures. 500,000 Monte Carlo samples of the material properties were used to produce these plots. Simulations were also ended if a critical maximum principal strain of $1 \%$ was reached. At $\mathrm{T}=550{ }^{\circ} \mathrm{C}$, as shown in the top row of Figure 4.4, none of the simulations reach the critical strain in 20 years. At this lower temperature, we also see that most of the material samples resulted in the lowest final strains and that only a low number of material samples demonstrated higher strains. The distribution of principal strains also increases with increase pressure. Note that the limits on the $\mathrm{x}$-axis Figure 4.4 are adjusted to fit each pressure's strain distribution. The strain distribution at the lowest pressure of $5 \mathrm{MPa}$ has a range of $1 \times 10^{-4}$ while at the $8 \mathrm{MPa}$ the range increases to $1 \times 10^{-3}$. Because of the logarithmic scale, some high strain outliers do not appear in the plots.

At $\mathrm{T}=600{ }^{\circ} \mathrm{C}$, as shown in Figure $4.4 \mathrm{~b}$, larger principal strain distributions are observed with all of the 
Final Maximum Principal Strain Distributions at 20 Years $(T=550 \mathrm{C})$

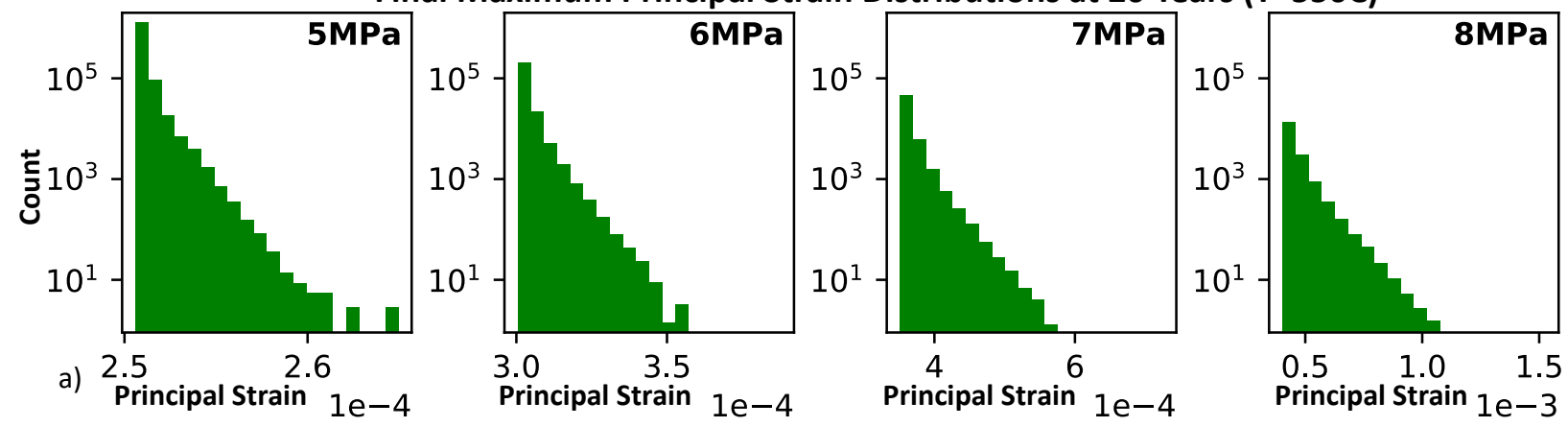

Final Maximum Principal Strain Distributions at 20 Years $(T=600 \mathrm{C})$

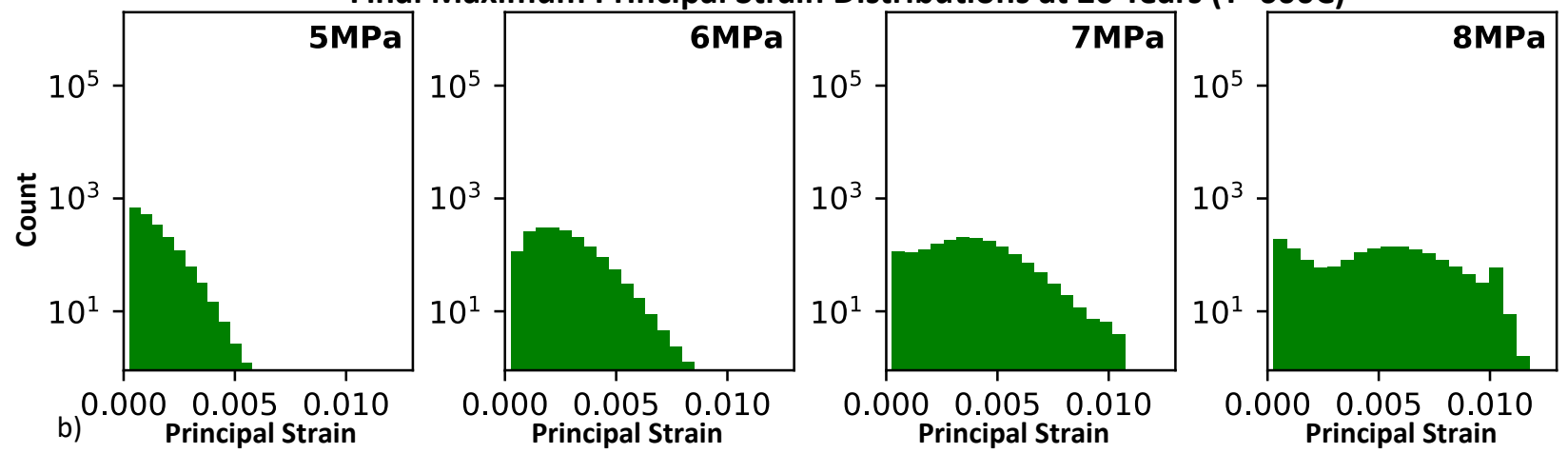

Figure 4.4: Final maximum principal strain reached after 20 years for 500,000 Monte Carlo samples of the posterior material distributions. The y-axis for all pressures and temperatures remains the same while in the top row, labeled (a), for $\mathrm{T}=550^{\circ} \mathrm{C}$ the $\mathrm{x}$-axis is adjusted to each pressure's distribution of maximum principal strain to account for the large variation. In the bottom row, labeled (b), for $\mathrm{T}=600{ }^{\circ} \mathrm{C}$, the larger distributions in strains allow us to use the same limits on the $\mathrm{x}$-axis for all pressures.

distributions, plotted with the same limits on the x-axis. In every distribution at least one sample of the material properties leads to a maximum principal strain above the critical level of $1 \%$. As the pressure increases at $\mathrm{T}=600{ }^{\circ} \mathrm{C}$, the distributions become larger and flatten out. The maximum strains at the higher pressures would continue to increase but the simulations were at the time step at which the critical strain exceeds $1 \%$, leading to the histograms reporting values slightly larger than $1 \%$. Some simulations reach strains slightly higher than $1 \%$ because the simulation is terminated on the next time step after the principal strain exceeds $1 \%$ strain.

The final strain states from Figure 4.4 are used to estimate a probability of failure at twenty years for a critical principal strain of $1 \%$. The probability of failure at twenty years is given by evaluating the cumulative density function $(\mathrm{CDF})$ for reaching this strain criterion at twenty years. For $\mathrm{T}=600^{\circ} \mathrm{C}$, the failure probability for $\mathrm{P}=6 \mathrm{MPa}$ is $1.2 \times 10^{-4}$, for $\mathrm{P}=7 \mathrm{MPa}$ it is $4.4 \times 10^{-3}$, and for $\mathrm{P}=8 \mathrm{MPa}$ it is $4.2 \times 10^{-2}$. Only a single sample reaches $1 \%$ strain for $\mathrm{T}=600{ }^{\circ} \mathrm{C}$ and $\mathrm{P}=5 \mathrm{MPa}$, making the probability of failure $2 \times 10^{-6}$ but more samples would be required to provide an accurate estimate of such a low probability of failure. None of the samples fail at $\mathrm{T}=550{ }^{\circ} \mathrm{C}$. The probability density function (PDF) and $\mathrm{CDF}$ for the time to failure are shown in Figure 4.5 for $\mathrm{T}=600{ }^{\circ} \mathrm{C}$ and two pressures; $6 \mathrm{MPa}$ and $7 \mathrm{MPa}$.

To correlate failure with the input material properties, we plot the distributions of the input parameters for the cases that failed in blue in Figure 4.6 and compare them to the total distribution of input parameters shown in green. We provide these plots for only the two pressures presented in Figure 4.5. We see that 

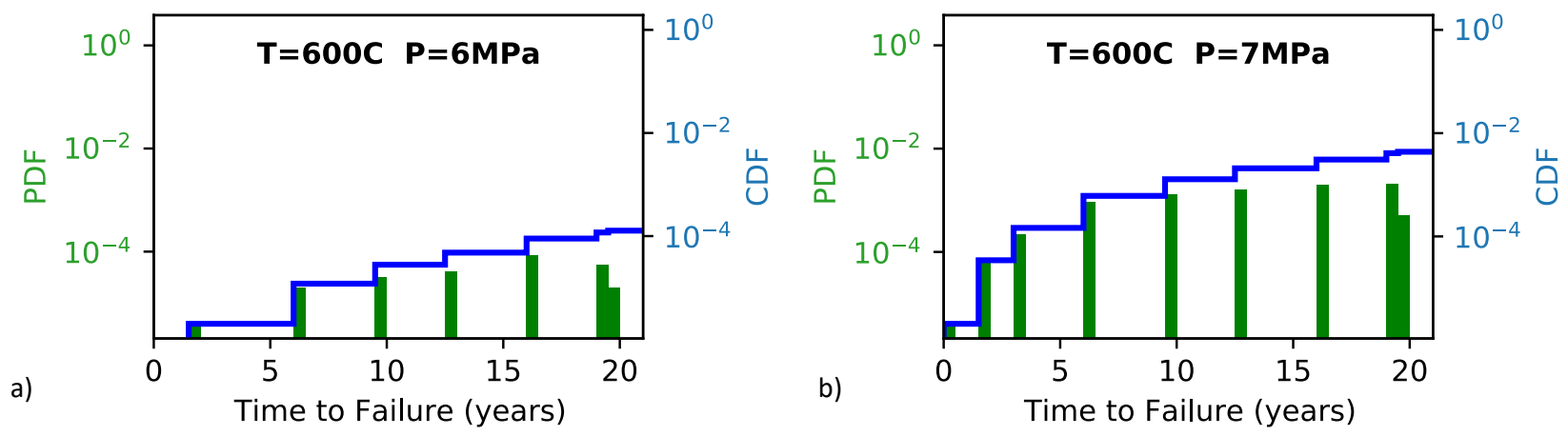

Figure 4.5: Probability density function (PDF, green histogram) and cumulative distribution functions (CDF, blue line) for simulations in Figure 4.4 that reach a critical maximum principal strain of $1 \%$ at $\mathrm{T}=600{ }^{\circ} \mathrm{C}$.

lower values for $n$ and $b$ are most closely correlated with failure, and that pressure does not significantly effect the shape of these two distributions for the failed samples. For the other material properties, the failed sample distributions closely match the original input parameter value distributions and thus do not have a large correlation with failure.

Since none of the samples reach a critical strain of $1 \%$ at any pressure for $\mathrm{T}=550{ }^{\circ} \mathrm{C}$, we choose an alternative critical strain criterion for plotting the distributions of parameters for "failed" cases: the top $1 \%$ of strain reached for all samples in Figure 4.3 is considered as the failure strain. For this adjusted criterion, we plot the distributions of material properties leading to the top $1 \%$ of strain in magenta in Figure 4.7 versus the original distributions of material properties in green. We only plotted the material distributions for the highest pressure tested, $\mathrm{P}=8 \mathrm{MPa}$, because we noted very little variation in the shapes of the material distributions that lead to the top $1 \%$ of final strains for all of the pressures tested. At the lower temperature we again see that $n$ leads to higher strains while the other material properties are shown to have very little effect. This pattern holds true even for $b$, which we saw was correlated with a higher probability of failure for $\mathrm{T}=600{ }^{\circ} \mathrm{C}$ in Figure 4.6. 


\section{Input Parameters $(\mathrm{T}=600 \mathrm{C}, \mathrm{P}=6 \mathrm{MPa})$}
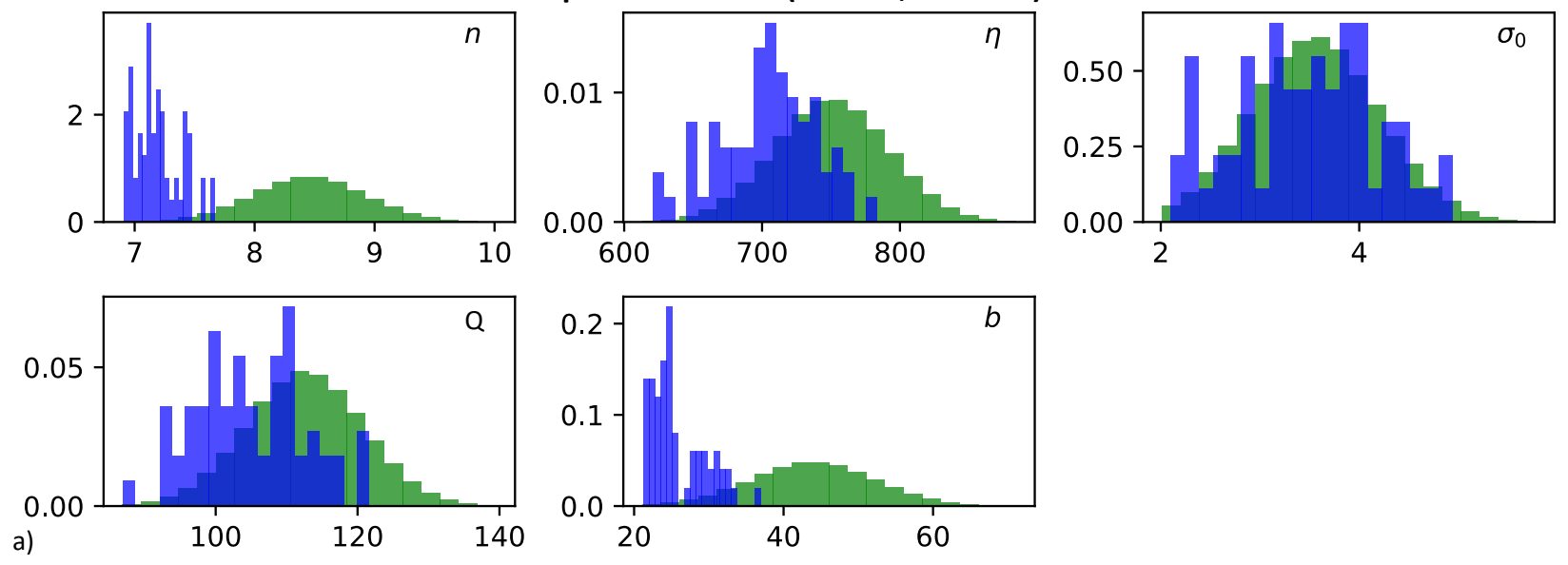

Input Parameters $(\mathrm{T}=600 \mathrm{C}, \mathrm{P}=7 \mathrm{MPa})$
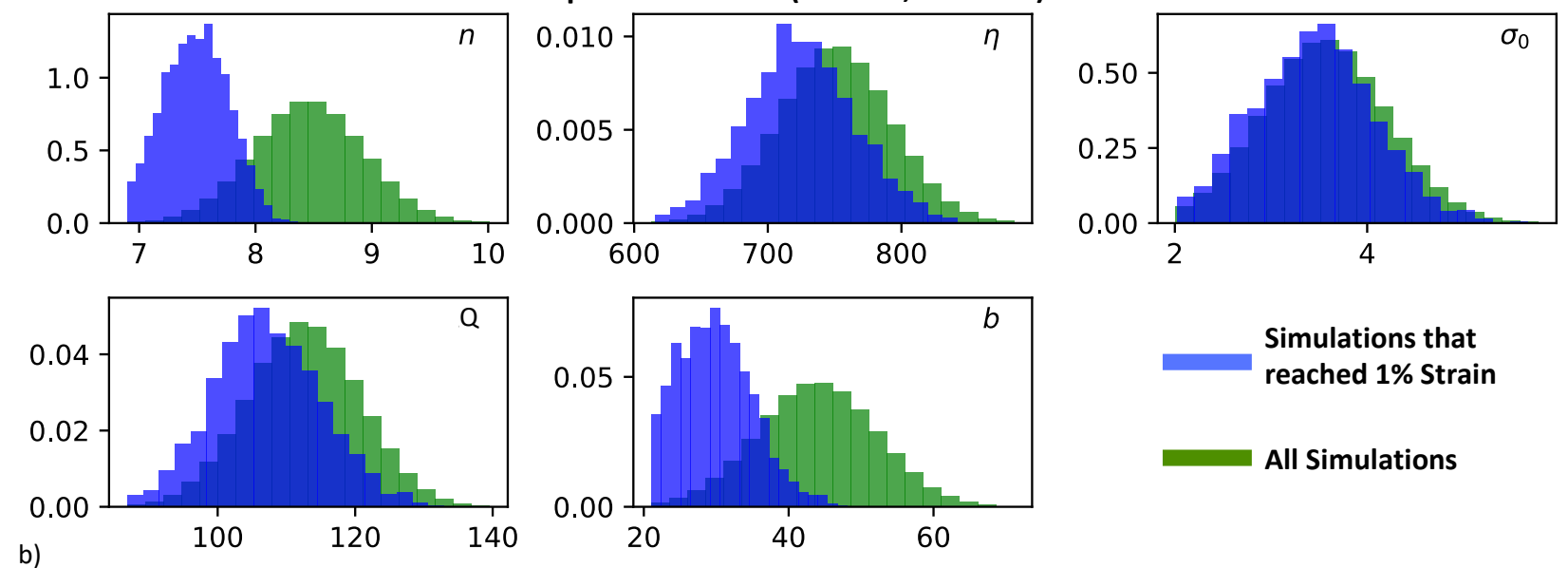

Simulations that reached $1 \%$ Strain

All Simulations

Figure 4.6: Normalized histograms of input material parameters for all 500,000 samples in green and those that failed within twenty years in blue at $\mathrm{T}=600^{\circ} \mathrm{C}$. The blue histogram in (a) contains 64 samples and (b) contains 2144 samples.

\section{Input Parameters ( $\mathrm{T}=550 \mathrm{C}, \mathrm{P}=\mathbf{8 \mathrm { MPa } )}$}
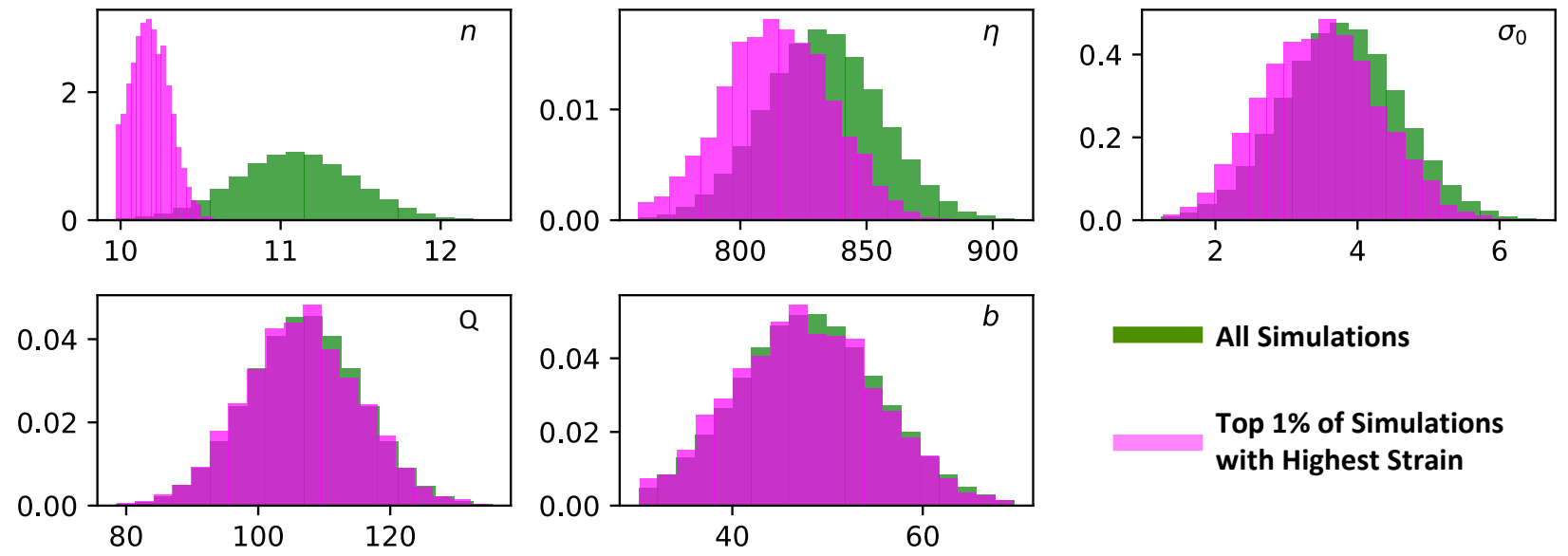

\section{All Simulations}

Top $1 \%$ of Simulations with Highest Strain

Figure 4.7: Normalized histograms of input material parameters for all 500,000 samples in green and the top $1 \%$ of samples with the highest strain after twenty years in magenta at $\mathrm{T}=550^{\circ} \mathrm{C}$ and $\mathrm{P}=8 \mathrm{MPa}$. 


\subsubsection{NEML Sphere Nozzle Model Results}

In this section we present the statistical results from the NEML Grade 91 material model applied to the pressurized sphere nozzle model from Figure 4.1 simulated at constant pressures and temperature. The stress and strain distribution for the sphere nozzle model, using the mean values of the material properties in Table 4.2 for $\mathrm{T}=600{ }^{\circ} \mathrm{C}$, is shown in Figure 4.8 with an applied internal pressure of $3 \mathrm{MPa}$. We see the stress and strain being concentrated near the junction of where the nozzle and pipe connect. We observe two locations near the sphere nozzle junction, on the inner and outer surface, where the von Mises stress and maximum principal strain reach their highest values. This simulation was terminated after 303 days when the critical strain of $1 \%$ was reached. The stress concentration in the nozzle sphere geometry leads to higher stresses than those observed in the simplified pipe model. For this reason, we perform our simulations over a lower, smaller pressure range of 1.5-3 $\mathrm{MPa}$ and the same temperatures.

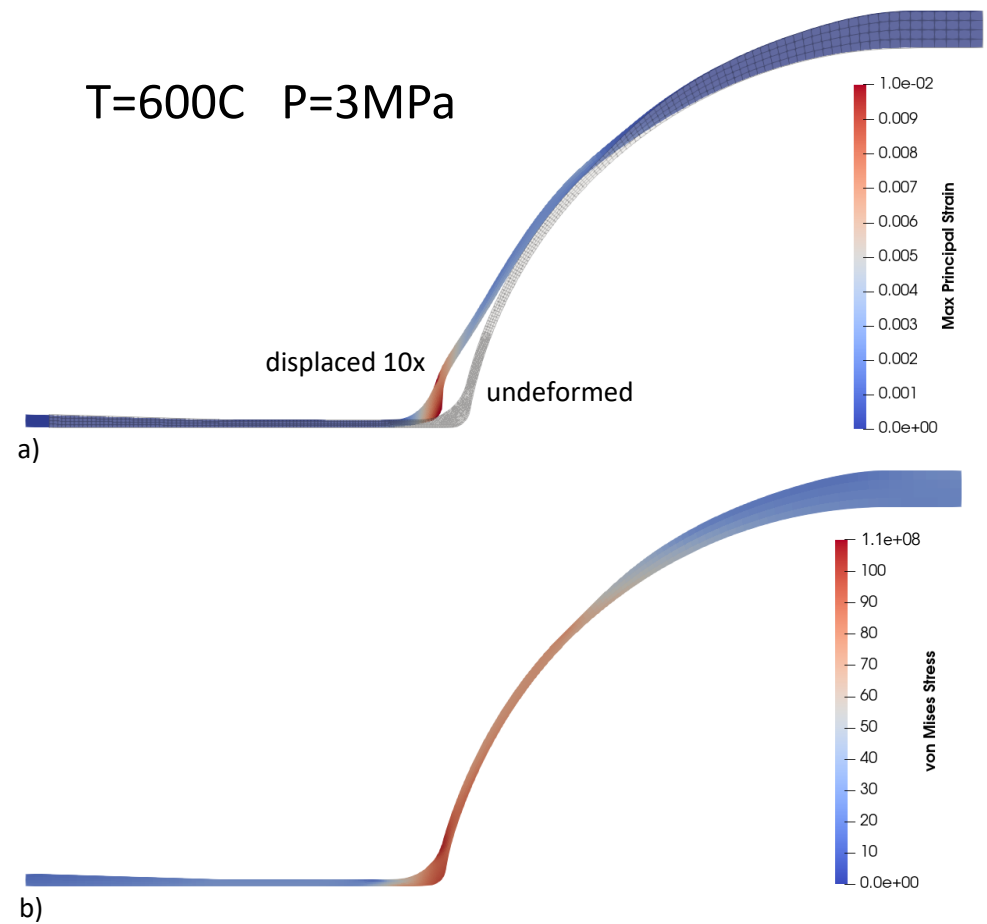

Figure 4.8: Constant pressure simulation results at $3 \mathrm{MPa}$ for mean NEML Grade 91 material properties at $\mathrm{T}=600{ }^{\circ} \mathrm{C}$ from Table 4.2 . The results shown are for a simulation time of 303 days at which point the maximum principal strain in one of the elements reached 1\%. (a) Final maximum principal strain contours on $10 \times$ displaced mesh, compared to the undeformed mesh in gray. (b). Final von Mises stress contours.

At each temperature and pressure we took 100,000 Monte Carlo samples from the truncated normal material distributions given in Table 4.2. The final states for the maximum principal strain at the end of 20 years for these simulations are shown in Figure 4.9. Overall the nozzle simulation results show similar behavior to that observed for the pipe model shown in Figure 4.4: none of the samples fail at $\mathrm{T}=550{ }^{\circ} \mathrm{C}$, the distributions at $\mathrm{T}=550{ }^{\circ} \mathrm{C}$ are clustered at low strain for each pressure and decrease exponentially toward higher strains, and increasing pressure increases the final strain distribution for both temperatures. Several of the simulations for $\mathrm{T}=600^{\circ} \mathrm{C}$ and pressures of $1.75,2$, and $2.5 \mathrm{MPa}$ were terminated early because they reached the critical strain of $1 \%$, causing the distributions to be artificially skewed toward $1 \%$ strain. Again, the simulations are terminated on the time step where the principal strain exceeds $1 \%$, allowing the maximum strain 
Final Maximum Principal Strain Distributions at 20 Years ( $T=550 \mathrm{C})$
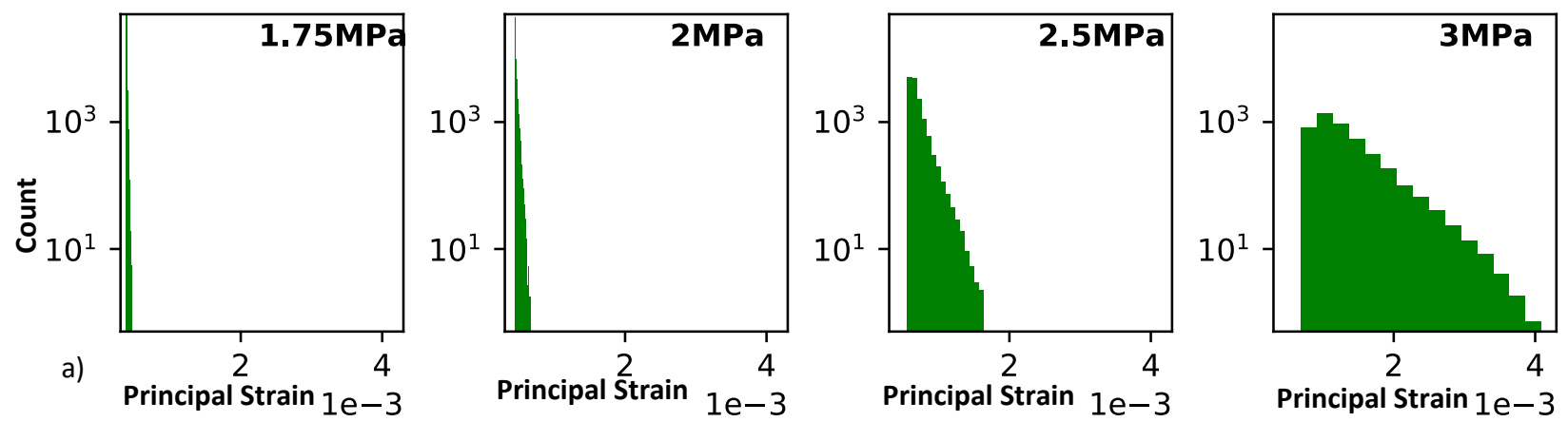

Final Maximum Principal Strain Distributions at 20 Years $(T=600 \mathrm{C})$
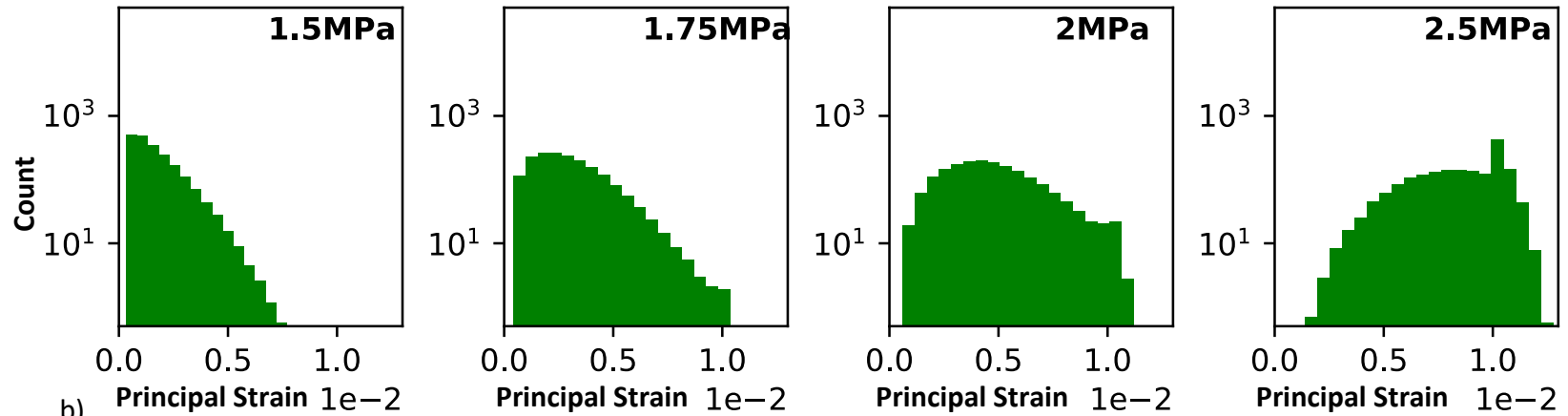

Figure 4.9: Final maximum principal strain reached after 20 years for 100,000 Monte Carlo samples of the posterior material distributions are compared to similar results given for the simplified pipe model in Figure 4.4. The y-axis for all pressures and temperatures remains the same. (a) In the top row for $\mathrm{T}=550{ }^{\circ} \mathrm{C}$, all pressures use the same $\mathrm{x}$-axis. (b) In the bottom row for $\mathrm{T}=600{ }^{\circ} \mathrm{C}$, the entire strain range from 0 to the critical strain of $1 \%$ is used for all pressures because of the large final strain distribution.

in some simulations to exceed $1 \%$.

The final strain states from Figure 4.9 are then used to estimate a probability of failure at twenty years for a critical principal strain of 1\%. The PDF and CDF for the time to failure are shown in Figure 4.10 for T=600 ${ }^{\circ} \mathrm{C}$ and two pressures; 1.75 and $2 \mathrm{MPa}$. The probability of failure at twenty years is given by evaluating the $\mathrm{CDF}$ for reaching this strain criterion at twenty years. For $\mathrm{T}=600^{\circ} \mathrm{C}$, the failure probability for $\mathrm{P}=1.75 \mathrm{MPa}$ is $1 \times 10^{-3}$ and for $\mathrm{P}=2 \mathrm{MPa}$ is $1.8 \times 10^{-2}$ and for $\mathrm{P}=2.5 \mathrm{MPa}$ is $3.5 \times 10^{-1}$. For $\mathrm{P}=3 \mathrm{MPa}$ and $\mathrm{T}=600{ }^{\circ} \mathrm{C}$, the conditions used for the simulation presented in Figure 4.8, the probability of failure is 0.89 , and it is not surprising that the simulation using mean material properties reached $1 \%$ strain.

In Figure 4.11, the material properties that resulted in simulations with principal strains above $1 \%$ for $\mathrm{P}=2$ $\mathrm{MPa}$ and $\mathrm{T}=600{ }^{\circ} \mathrm{C}$ are shown in blue compared to the entire distribution of material properties in green. As was previously observed in the pipe model, lower values of $n$ and $b$ are seen to be most closely correlated with the simulations that reach the critical principal strain. The low probability of failure and the small number of samples for $\mathrm{P}=1.75 \mathrm{MPa}$ and $\mathrm{T}=600{ }^{\circ} \mathrm{C}$ results in only about 100 simulations failing, resulting in a much sparser, noisier distribution that that of the full set of samples. However, this distribution does show similar trends to those observed in Figure 4.11. As for the simple pipe model, the other material properties do not have a strong correlation with failure, even with the more complicated stress distribution in this model.

We do not observe any failed samples at any pressure for $\mathrm{T}=550{ }^{\circ} \mathrm{C}$, so again we choose the same alternative critical strain criterion used in the pipe simulations as the top $1 \%$ of strain reached for all samples in 


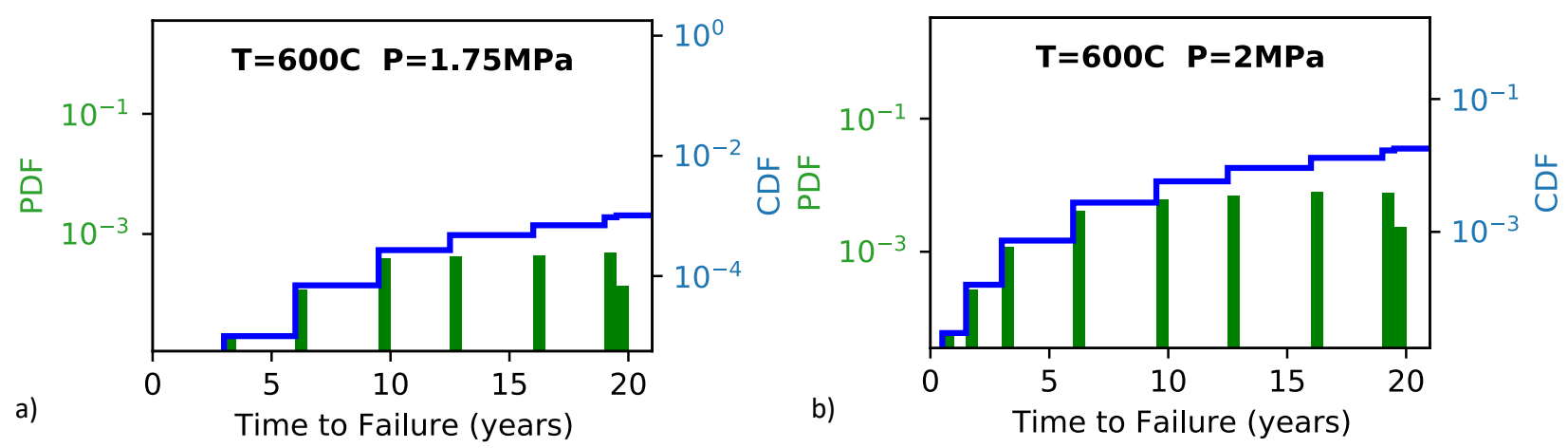

Figure 4.10: Probability density function (green histogram) and cumulative distribution functions (blue line) for simulations in Figure 4.5 that reach a critical maximum principal strain of $1 \%$ at $\mathrm{T}=600{ }^{\circ} \mathrm{C}$.

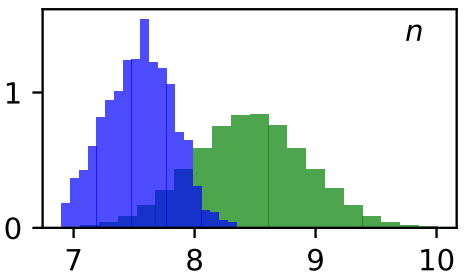

Input Parameters $(\mathrm{T}=600 \mathrm{C}, \mathrm{P}=\mathbf{2} \mathrm{MPa})$
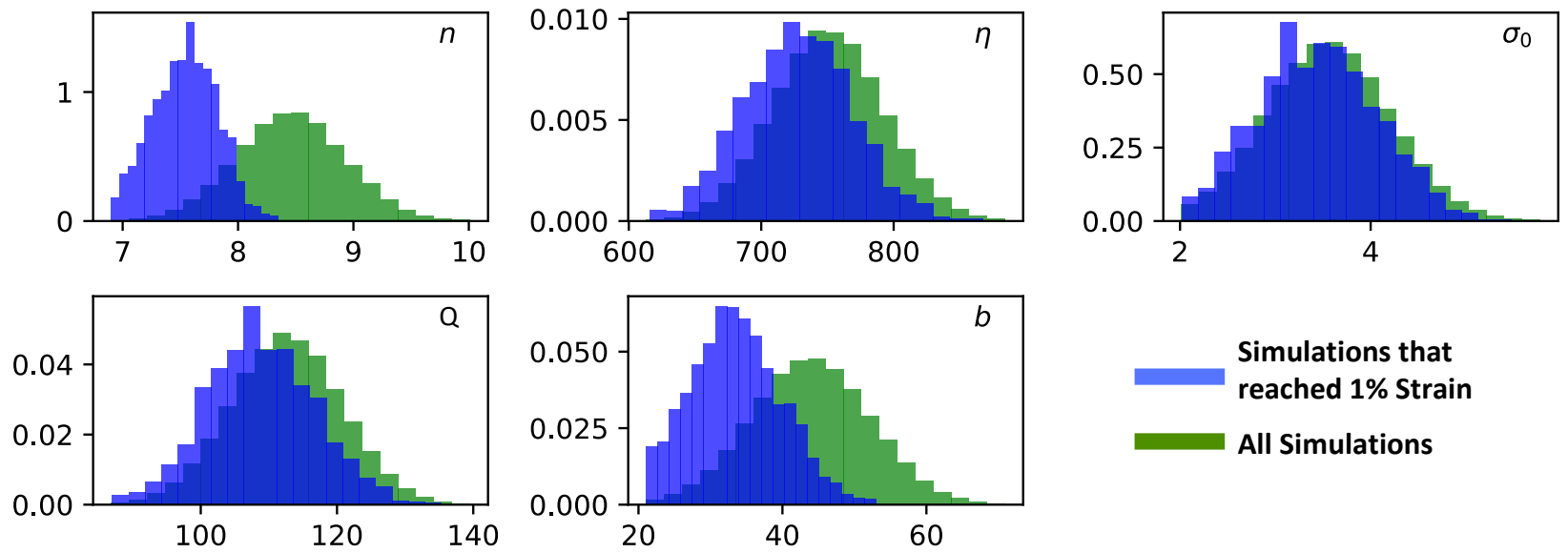

Simulations that reached $1 \%$ Strain All Simulations

Figure 4.11: Normalized histograms of input material parameters for all 100,000 samples in green and those that failed within twenty years in blue at $\mathrm{T}=600^{\circ} \mathrm{C}$. The blue histogram contains 1797 samples. 
Figure 4.9. For this criterion, we plot the distributions of material properties leading to the top $1 \%$ of strain in magenta in Figure 4.12 versus the original distributions of material properties in green. The distribution of material properties leading to failure in Figure 4.12 are for $\mathrm{P}=2.5 \mathrm{MPa}$ and are representative of the other pressures simulated at $\mathrm{T}=550{ }^{\circ} \mathrm{C}$. The material property distribution most closely correlated to high principal strains for all pressures and temperatures with the NEML Grade 91 material model is $n$. We observed that $b$ only affects the strain at the higher temperature of $\mathrm{T}=600^{\circ} \mathrm{C}$. These observations were true for both models, so the complexity of the strain distribution does not appear to make a difference.

Input Parameters ( $\mathrm{T}=550 \mathrm{C}, \mathrm{P}=\mathbf{2} .5 \mathrm{MPa})$
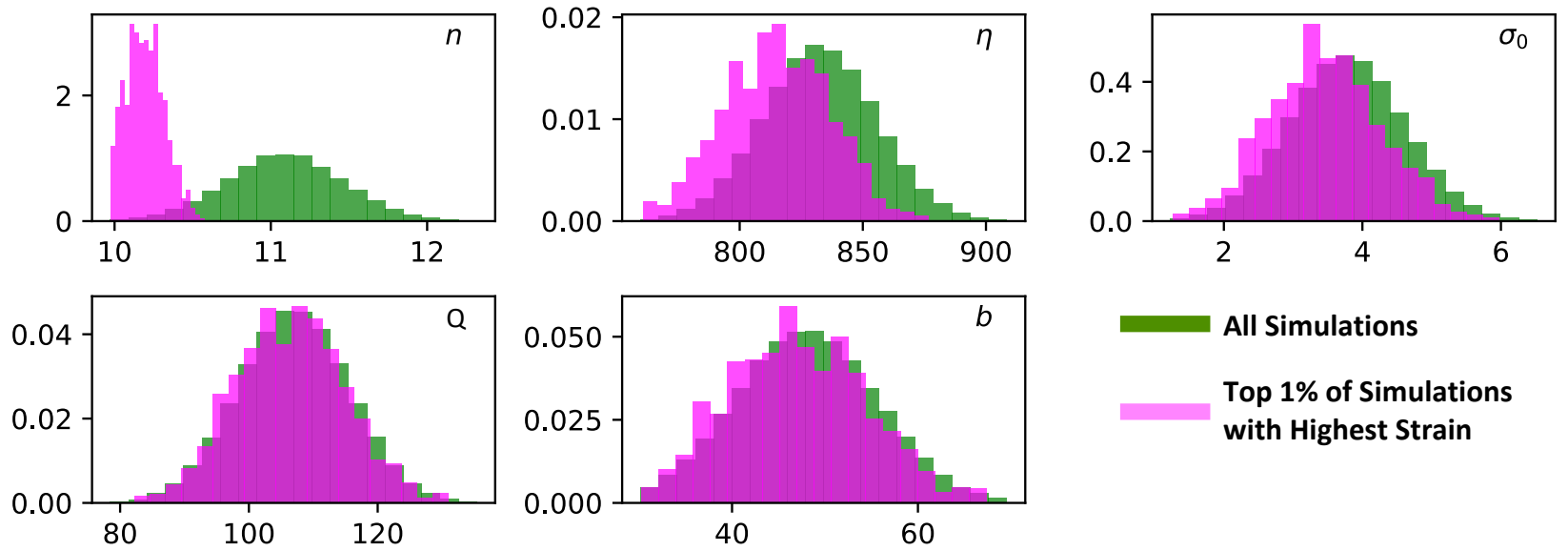

All Simulations

Top 1\% of Simulations with Highest Strain

Figure 4.12: Normalized histograms of input material parameters for all 100,000 samples in green and the top $1 \%$ of samples with the highest strain after twenty years in magenta at $\mathrm{T}=550{ }^{\circ} \mathrm{C}$ and $\mathrm{P}=2.5$ MPa. Similar trends were observed for $\mathrm{P}=1.75,2$, and $3 \mathrm{MPa}$.

\subsection{LAROMANCE Material Model Results}

In this section, the LAROMANCE material model developed for Grade 91 is used to model the simplified pipe and nozzle sphere shown in Figure 4.1 over a range of material inputs for initial cell and initial wall dislocation densities and MX precipitate phase content. We use the same loading and boundary conditions as used for the evaluation of the NEML Grade 91 material model in the previous section. For consistency between the NEML and LAROMANCE Grade 91 material model simulations, the same initial elastic constants given in Table 4.1 are used here. Truncated normal distributions are used to define the LAROMANCE P91 input material parameters and are given in Table 4.3. Values defining these distributions were chosen to span the range of the reduced order model's applicability given in Table 3.2, except for the maximum MX phase, which we limit to $\phi_{M X}=0.02$. The same distributions for initial material properties are used for both temperatures; $550{ }^{\circ} \mathrm{C}$ and $600{ }^{\circ} \mathrm{C}$. All of our simulations assume homogeneous material properties at the sampled values for the entire model.

Table 4.3: LAROMANCE material property distributions from Table 3.2.

\begin{tabular}{|c|c|c|}
\hline parameter & $(\mu, \sigma)$ & Bounds \\
\hline Cell Dislocation Density $\left(10^{12} \mathrm{~m}^{-2}\right)$ & $(4,1)$ & {$[1,6]$} \\
Wall Dislocation Density $\left(10^{12} \mathrm{~m}^{-2}\right)$ & $(12,3)$ & {$[6,18]$} \\
MX Phase Fraction $\left(\phi_{M X}\right)$ & $(0.012,0.003)$ & {$[0.006,0.02]$} \\
\hline
\end{tabular}




\subsubsection{LAROMANCE Simplified Pipe Model Results}

For the simplified pipe model, the maximum principal strain as a function of time is plotted in Figure 4.13 together with the dislocation density evolution for two different temperatures and MX phase contents, $\phi_{M X}$. From the large number of simulations completed for the following analysis, we found that the final strain response was most sensitive to $\phi_{M X}$. For these simulations we we selected values of $\phi_{M X}$ slightly lower than the mean $\left(\phi_{M X}=0.008\right)$ and at at the upper cut-off in Table $4.3\left(\phi_{M X}=0.02\right)$. At the lower temperature of $\mathrm{T}=550^{\circ} \mathrm{C}, \phi_{M X}$ has almost no effect on the strain evolution and only has a slight effect on the evolution of the cell dislocation density. However at $\mathrm{T}=600^{\circ} \mathrm{C}$, the higher $\phi_{M X}$ value results in a larger principal strain and a smaller decrease in cell dislocation density.

Increasing precipitate size can either increase or decrease creep lifetime in ductile metals [2]. Beneath a critical precipitate size, increasing precipitate sizes have been observed to lower creep rates. Above the critical precipitate size this trend reverses, and larger MX precipitates instead increase steady-state creep rates. Chaturvedi and Han [2] referred to microstructures as under- or overaged in relation to this critical precipitate size. Comeli et al. [3] found the middle of three annealing temperatures to result in the lowest minimum creep rate in a CrMoV steel with precipitate content similar to that of P91. In general, these precipitates act as obstacles to dislocation glide, typically reducing creep rates. At high temperature creep conditions, dislocations must bypass precipitates via dislocation climb or by bowing out between obstacles. As precipitate size increases, the bowing-out mechanism becomes more dominant. In addition, dislocation annihilation occurs during dislocation creep, depleting dislocations during the transition from primary to secondary creep. If dislocation annihilation is prevented during primary creep, a larger density of dislocations would exist during secondary creep. The thermally assisted motion of more dislocations would in turn lead to higher creep rates in the secondary creep regime. This is consistent with the creep rates in P92 steel observed by Han et al. [4], for which the longest aging led to the highest steady-state creep rate and a reduced initial creep rate.

In this section, we carry out an analysis similar to that completed for the NEML material model. Again, we take 500,000 Monte Carlo samples over the range of input material properties provided in Table 4.3. The final maximum principal strain from these samples is shown in Figure 4.14 for a simulation time of 20 years at two temperatures; $550{ }^{\circ} \mathrm{C}$ and $600{ }^{\circ} \mathrm{C}$, and at four pressures ranging from 5-7 MPa. These simulations were run for either 20 years or until a critical strain level of $0.5 \%$ was reached. The LAROMANCE and NEML material models are fit to very different types of experimental data, leading to different predicted responses under similar loading conditions. Overall, the LAROMANCE model predicts lower strains with less variability than the NEML model. For this reason, we reduced the critical strain measure from $1 \%$ to $0.5 \%$ in order to differentiate the samples that are more likely to fail first. Comparing Figure 4.14 (a) and (b), we see that as temperature increases, so does the spread in total principal strain. We also note that as pressure increases, at each temperature, the strain also increases. The critical strain cutoff value explains the spikes in the failures at $0.5 \%$ strain in Figure 4.14 for $\mathrm{T}=600{ }^{\circ} \mathrm{C}$ and two pressures; $6.5 \mathrm{MPa}$ and $7 \mathrm{MPa}$.

At the lower temperature and lower pressures, no simulations reach the critical strain level of $0.5 \%$. To analyze the effect of initial material properties on the resulting levels of strain, we again use an alternative critical strain measure to filter our results. As was previously done, we define the alternative critical strain as the top $1 \%$ of strains reached for all samples, and we plot the material properties leading to these high strains in magenta in Figure 4.15. Figure 4.15 (a) shows that at $550{ }^{\circ} \mathrm{C}$, maximum strain is strongly correlated with a high initial dislocation content in the cells and a low obstacles content in the cell walls and precipitates. In addition, as we had discussed, with increasing temperature the relative effect of precipitate strengthening is more complex. Indeed, at $600{ }^{\circ} \mathrm{C}$, shown in Figure 4.15 (b), an increase in precipitate content does not necessarily reduce the maximum strain achieved. This is due to the fact that if the dislocations can easily glide at the onset of loading they will annihilate much faster (this process is thermally activated) such that as time passes, there will be fewer dislocations available to carry out plastic work, leading to a smaller total 

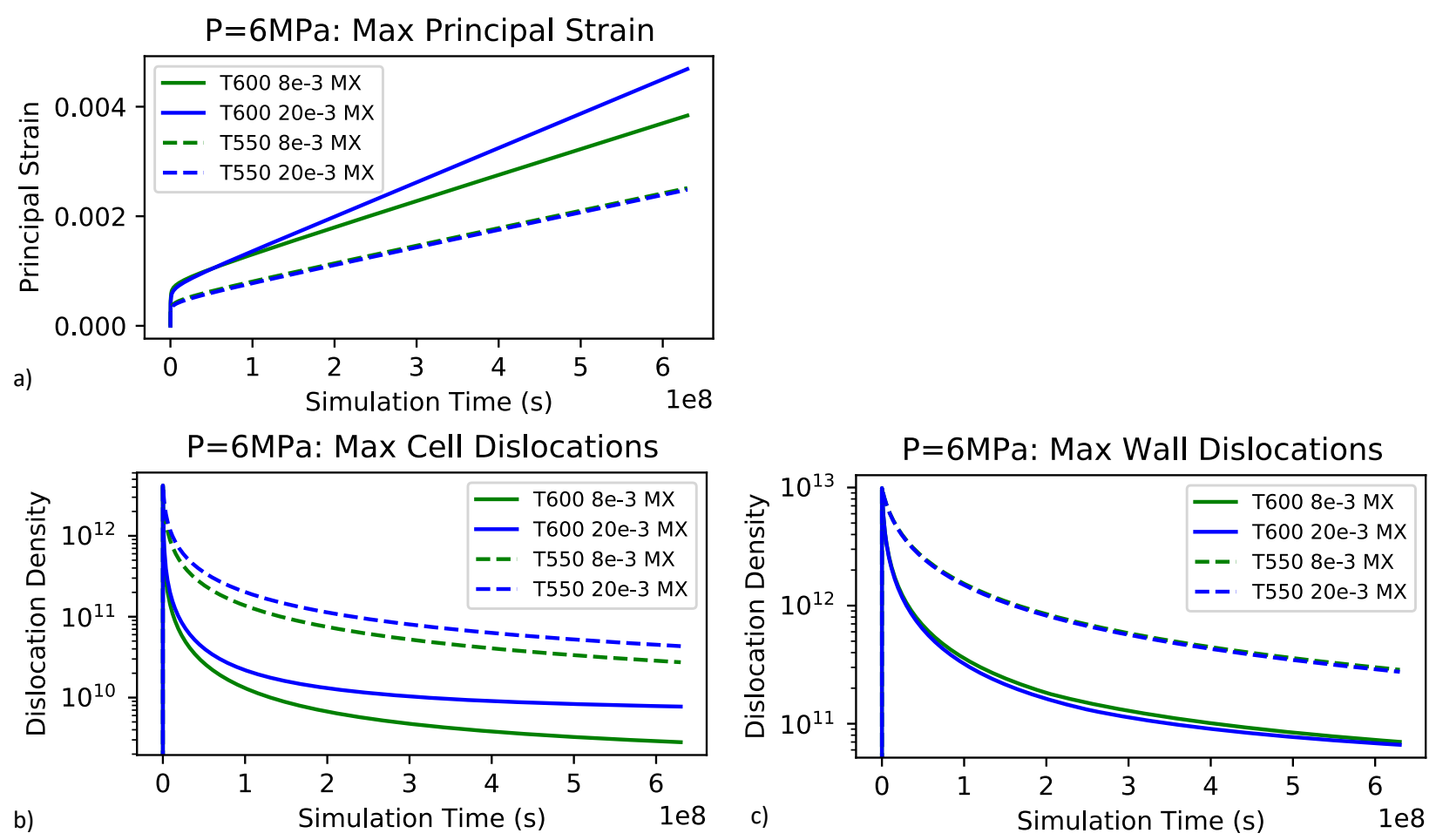

Figure 4.13: (a) Maximum principal strain and (b) cell and (c) wall dislocation density evolution as a function of simulation time for two different temperatures and MX phase content.

strain than that reached with a higher initial precipitate content.

The annihilation of dislocations over the course of the simulation is shown by the two-orders-of-magnitude reduction between the initial dislocation densities in Figure 4.15 and the final values in Figure 4.16. We note that at $\mathrm{T}=600{ }^{\circ} \mathrm{C}$ in Figure 4.16 (b), the simulations reaching the highest strains are those with the highest final cell dislocation densities. We did not see this same strong correlation between the initial cell dislocation density and high strains in Figure 4.15 (b). This observation reinforces the fact that a higher precipitate content inhibits dislocations from annihilating, making them available for creep deformation. The large change in correlation between the initial and final dislocation density and high strains indicates that the reduction in dislocation annihilation due to precipitates more than makes up for an initially low cell dislocation density.

The final strain states from Figure 4.14 are also used to estimate a probability of failure at twenty years for a critical strain of $0.5 \%$. The probability density function and cumulative distribution function for the time to failure are shown in Figure 4.17 for $\mathrm{T}=600{ }^{\circ} \mathrm{C}$ and two pressures; $6.5 \mathrm{MPa}$ and $7 \mathrm{MPa}$. The probability of failure at 20 years for $\mathrm{P}=6.5 \mathrm{MPa}$ is 0.11 and for $\mathrm{P}=7 \mathrm{MPa}$ it is 0.82 .

To correlate failure of the model with the input material properties, we plot the distributions of input parameters that lead to failure in blue in Figure 4.18 and compare them to the total distribution of input parameters shown in green. We see that high values for $\phi_{M X}$ are most closely correlated with failure. Even at $\mathrm{P}=7 \mathrm{MPa}$ where almost $80 \%$ of samples failed, we see that a low $\phi_{M X}$ is correlated with those samples that do not fail.

In Figure 4.18, the initial dislocations densities are not as strongly correlated to large strains as the precipitate content and it is difficult to tell what role it plays in this set of simulations. A stronger correlation may have been achieved by holding the precipitate content constant and varying only the initial dislocation density. The final wall and cell dislocation densities in Figure 4.19 show the evolution of dislocations from their 
Final Maximum Principal Strain Distributions at 20 Years ( $T=550 \mathrm{C})$
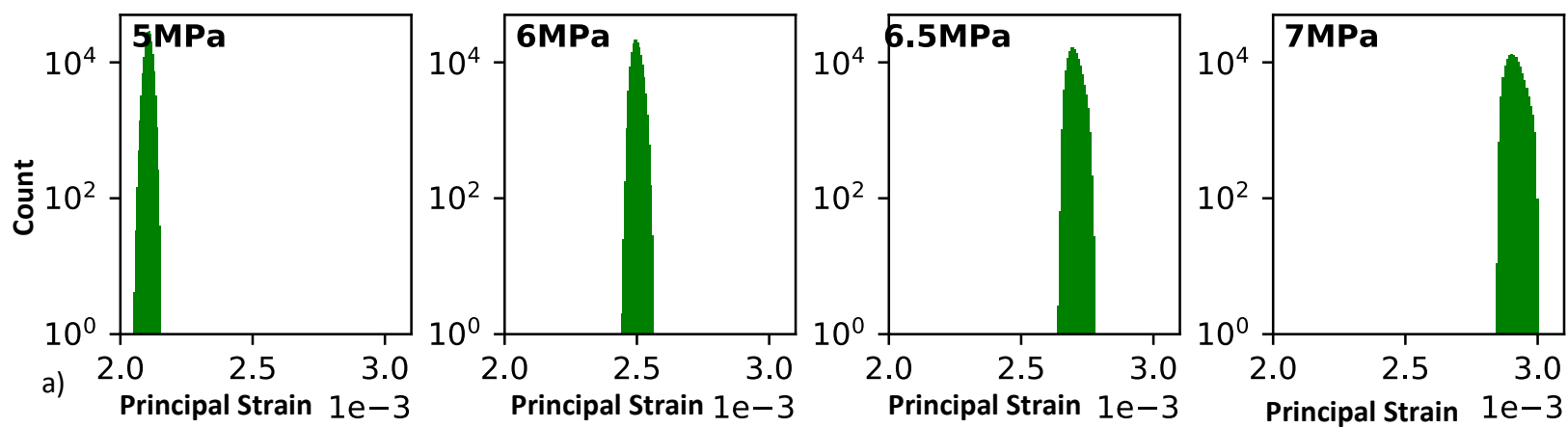

Final Maximum Principal Strain Distributions at 20 Years $(T=600 \mathrm{C})$
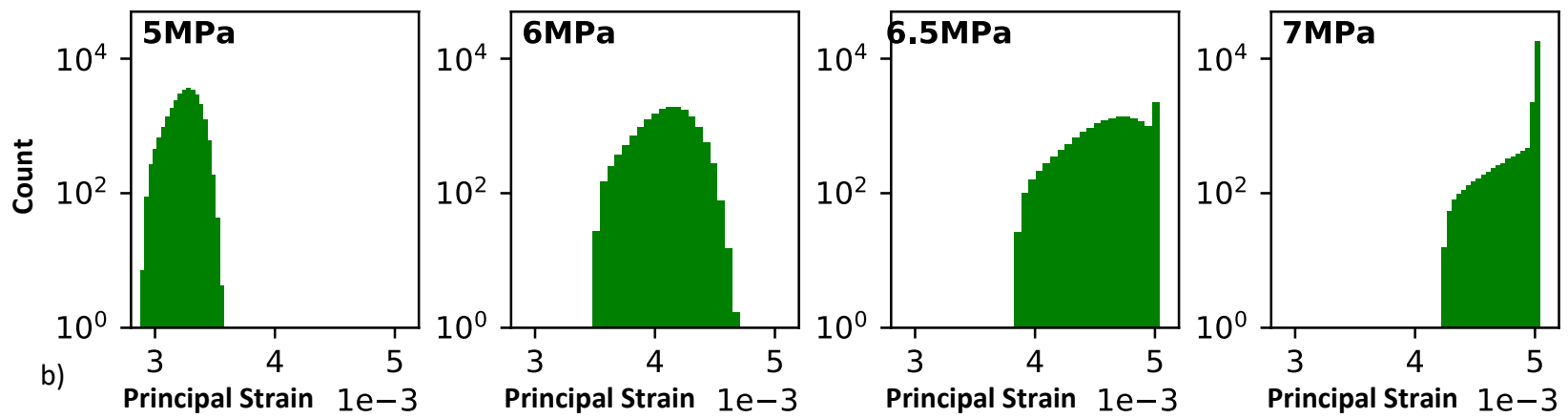

Figure 4.14: Final maximum principal strain reached after 20 years for 500,000 Monte Carlo samples of the material distributions with in the range of properties the LAROMANCE model was fit for temperatures (a) $550{ }^{\circ} \mathrm{C}$ and (b) $600{ }^{\circ} \mathrm{C}$.

\section{Input Parameters ( $\mathrm{T}=550 \mathrm{C}, \mathrm{P}=6 \mathrm{MPa})$}
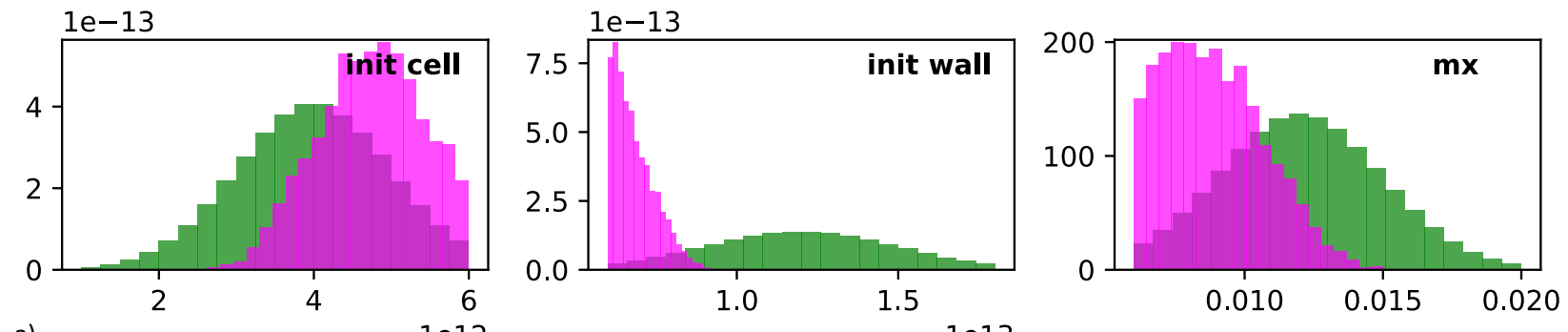

a)
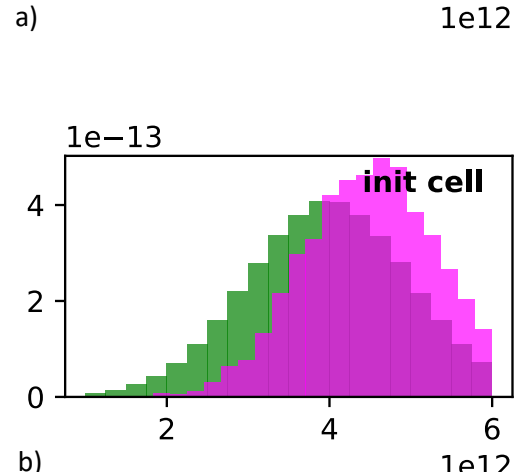

Input Parameters $(\mathrm{T}=600 \mathrm{C}, \mathrm{P}=6 \mathrm{MPa})$

All Simulations
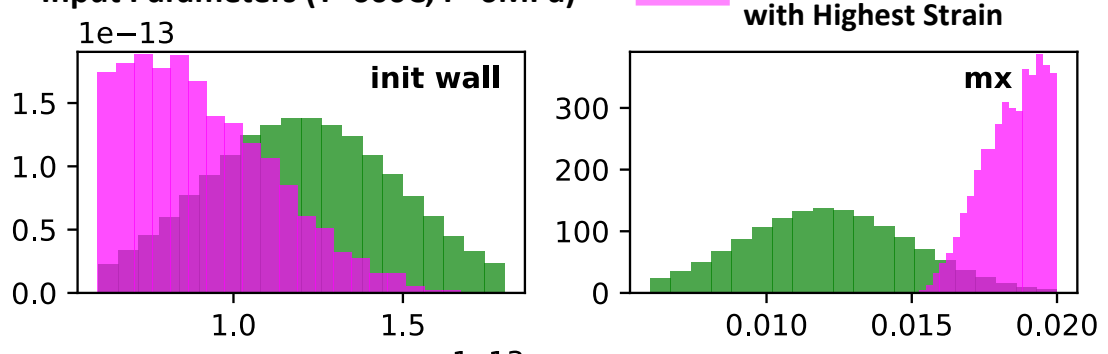

Figure 4.15: Normalized histograms of input material parameters for all 500,000 samples in green and those with the top $1 \%$ of strain in magenta for $6 \mathrm{MPa}$ and temperature (a) $550{ }^{\circ} \mathrm{C}$ and (b) $600{ }^{\circ} \mathrm{C}$. 
Input Parameters $(\mathrm{T}=550 \mathrm{C}, \mathrm{P}=6 \mathrm{MPa})$

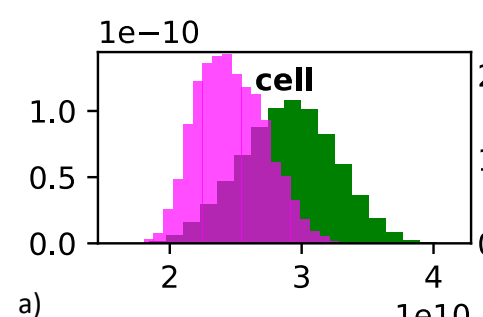

a)

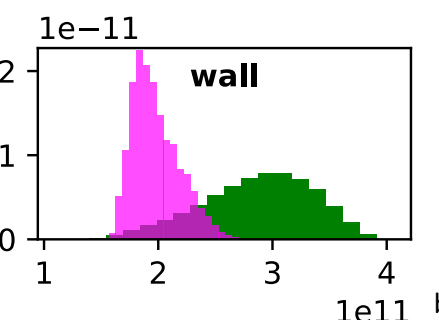

Input Parameters $(\mathrm{T}=600 \mathrm{C}, \mathrm{P}=6 \mathrm{MPa})$

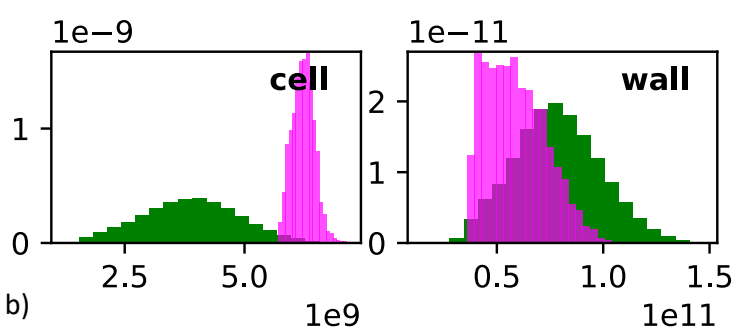

Top 1\% of Simulations with Highest Strain

Figure 4.16: Normalized histograms of the final wall and cell dislocation density evolution for all 500,000 samples in green and those with the top $1 \%$ of strain in magenta for $6 \mathrm{MPa}$ and temperature (a) $550{ }^{\circ} \mathrm{C}$ and (b) $600{ }^{\circ} \mathrm{C}$.
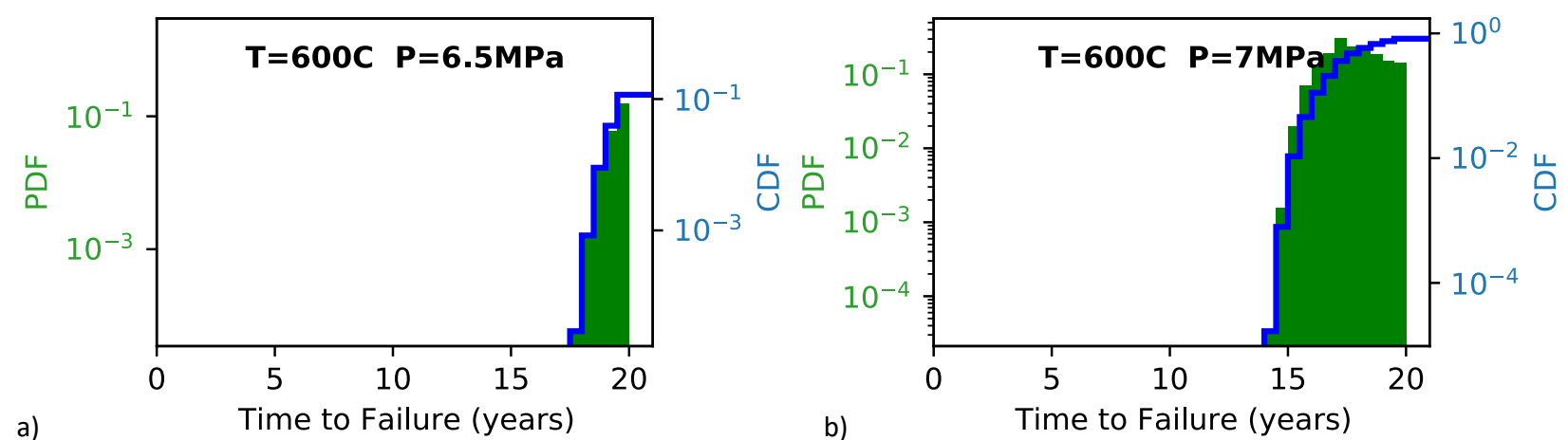

Figure 4.17: Normalized probability density function (green histogram) and cumulative distribution functions for simulations in Figure 4.14 that reach a critical maximum principal strain of $0.5 \%$ at $\mathrm{T}=600{ }^{\circ} \mathrm{C}$ for pressures (a) $6.5 \mathrm{MPa}$ and (b) $7 \mathrm{MPa}$. 
Input Parameters $(\mathrm{T}=600 \mathrm{C}, \mathrm{P}=6.5 \mathrm{MPa})$
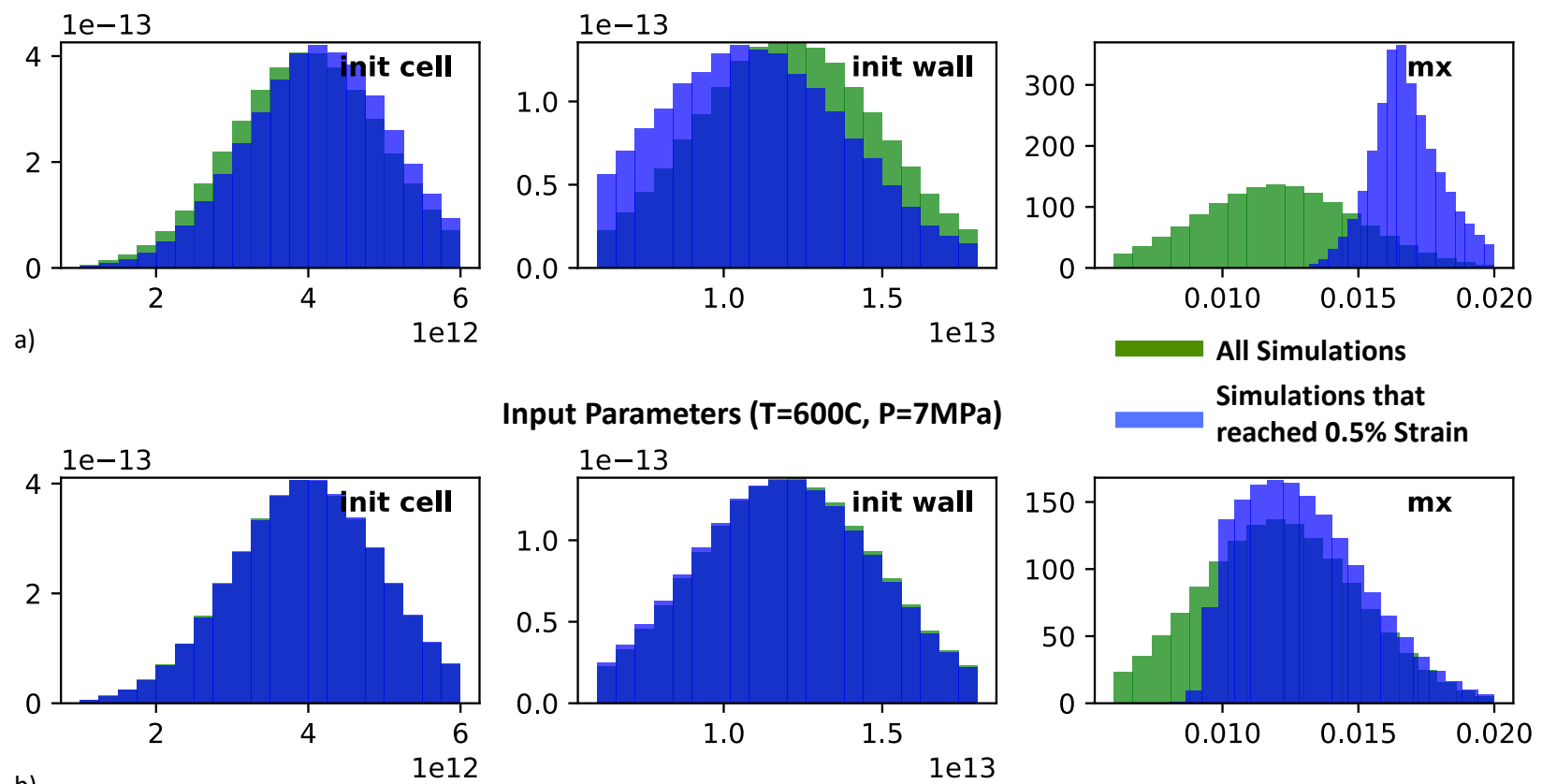

Input Parameters $(\mathrm{T}=600 \mathrm{C}, \mathrm{P}=7 \mathrm{MPa})$
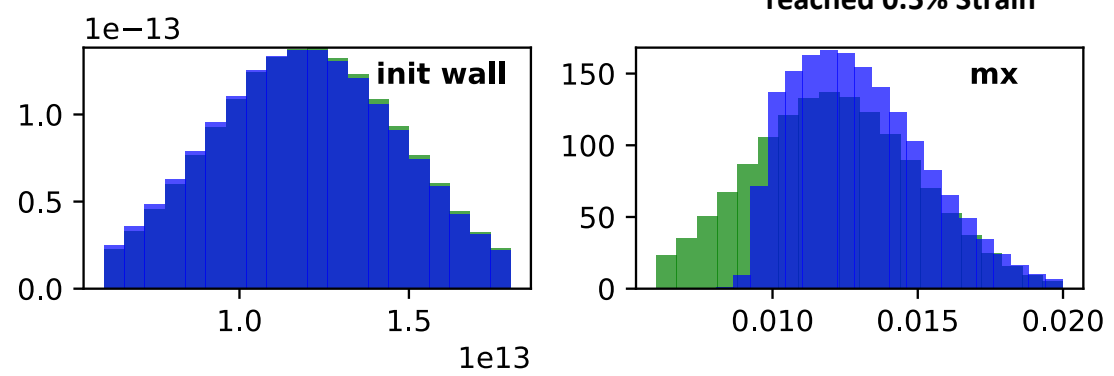

Figure 4.18: Normalized histograms of input material parameters for all 500,000 samples in green and those that failed within twenty years in blue at $\mathrm{T}=600{ }^{\circ} \mathrm{C}$. The blue histogram in (a) for $\mathrm{P}=6.5 \mathrm{MPa}$ contains 56,000 samples and (b) for $\mathrm{P}=7 \mathrm{MPa}$ contains 390,000 samples.

initial distributions shown in Figure 4.18, where the blue distributions are from the samples that reached 0.5\% strain and the green distributions are for all of the samples. For every sample, the cell and wall dislocation density are reduced by 2 orders of magnitude over 20 years for every pressure and temperature simulated. As previously mentioned, the initial dislocation densities shown in Figure 4.18 are not strongly correlated with a high strain; however, a higher final cell dislocation density is shown to be strongly correlated failure and with a high $\phi_{M X}$. 


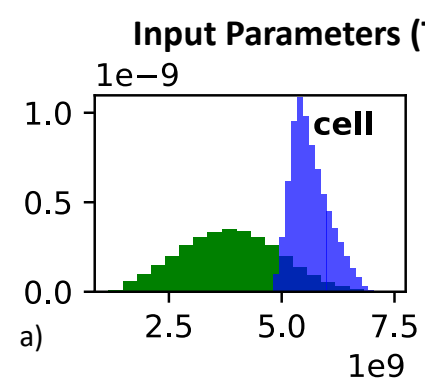

$(\mathrm{T}=600 \mathrm{C}, \mathrm{P}=6.5 \mathrm{MPa})$

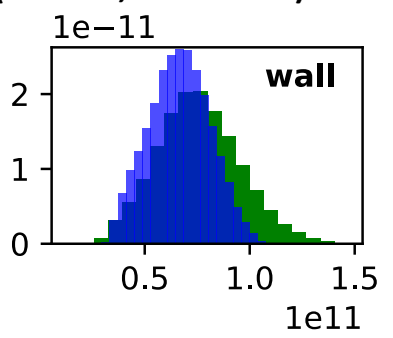

All Simulations
Input Parameters ( $\mathrm{T}=600 \mathrm{C}, \mathrm{P}=7 \mathrm{MPa})$

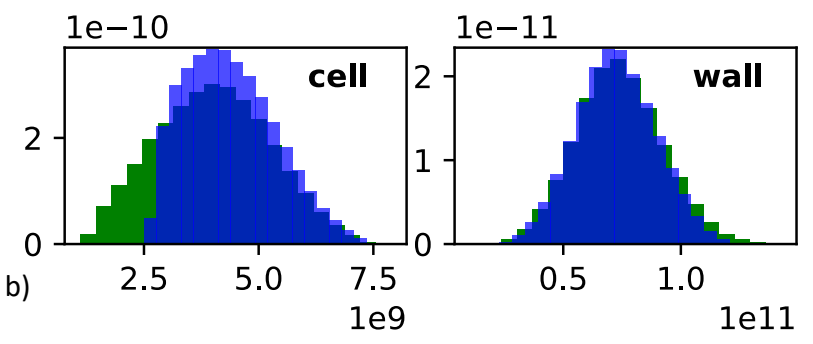

Simulations that

reached $0.5 \%$ Strain

Figure 4.19: Normalized histograms of the final wall and cell dislocation density evolution for all 500,000 samples in green and those that failed within twenty years in blue at $\mathrm{T}=600{ }^{\circ} \mathrm{C}$. The blue histogram in (a) contains 56,000 samples and (b) contains 390,000 samples.

\subsubsection{LAROMANCE Sphere Nozzle Model Results}

In this section the LAROMANCE Grade 91 material model is applied to the pressurized sphere nozzle model from Figure 4.1. We observed the LAROMANCE model to be most sensitive to MX phase content for the simplified pipe model in the previous section. This sensitivity was observed in the strain history in Figure 4.13 as well as the histogram of material properties leading to a critical strain in Figure 4.17. In this section we will use the same analysis procedure to determine the sensitivity of the sphere nozzle model to input material properties and determine if the more complicated model follows the same trends observed in the pipe model.

We begin by first plotting the evolution of maximum principal strain and dislocation densities over the duration of a twenty year simulation for two temperatures, $\mathrm{T}=550^{\circ} \mathrm{C}$ and $600^{\circ} \mathrm{C}$ and two $\mathrm{MX}$ phase contents, $\phi_{M X}=8 \times 10^{-3}$ and $20 \times 10^{-3}$. We notice the same trend as previously observed in Figure 4.19: higher $\phi_{M X}$ leads to larger strains at $\mathrm{T}=600{ }^{\circ} \mathrm{C}$. At the lower temperature of $\mathrm{T}=550^{\circ} \mathrm{C}, \phi_{M X}$ does not have an effect on the strain evolution as was also noted in the pipe model. The dislocation densities also evolve in a similar manner to that observed for the pipe model, where the higher $\phi_{M X}$ leads to higher cell dislocation densities and does not affect the density of wall dislocations.

The stress and strain distribution for the sphere nozzle model using the mean values of the material properties in Table 4.3 for $\mathrm{T}=600{ }^{\circ} \mathrm{C}$ are shown in Figure 4.21 with an applied internal pressure of $2 \mathrm{MPa}$. As expected, the stress and strain are concentrated near the transition of the nozzle into the sphere. In the closeup view of the transition region, we see that the maximum principal strain value occurs on the inner surface near the corner while the maximum von Mises stress occurs at two locations on the inner and outer surface. This is a much more complicated stress state than that created by the pipe model, where the maximum von Mises stress is always at the inner surface and decreases monotonically through the thickness. The final dislocation densities are plotted in Figure 4.22, and we see that the regions with the highest von Mises stress have the lowest dislocation density. We also note that the contours for both dislocation densities are similar in appearance, although the cell dislocation density is over an order of magnitude smaller than the wall dislocation density. This simulation was terminated after 11.9 years when the critical strain of $0.5 \%$ was reached.

The LAROMANCE Grade 91 model, which has a unique ability to capture the temperature dependent precipitate strengthening and dislocation evolution, comes at an increase in computational cost relative to the NEML Grade 91 material model. Due to this increased run time, only 4800 samples of the sphere nozzle model were simulated here. In Figure 4.23 we plot the final strain distributions at both temperatures at two different pressures at each temperature. For $\mathrm{T}=550{ }^{\circ} \mathrm{C}$ we see no samples reaching $0.5 \%$ strain for $\mathrm{P}=2 \mathrm{MPa}$ 

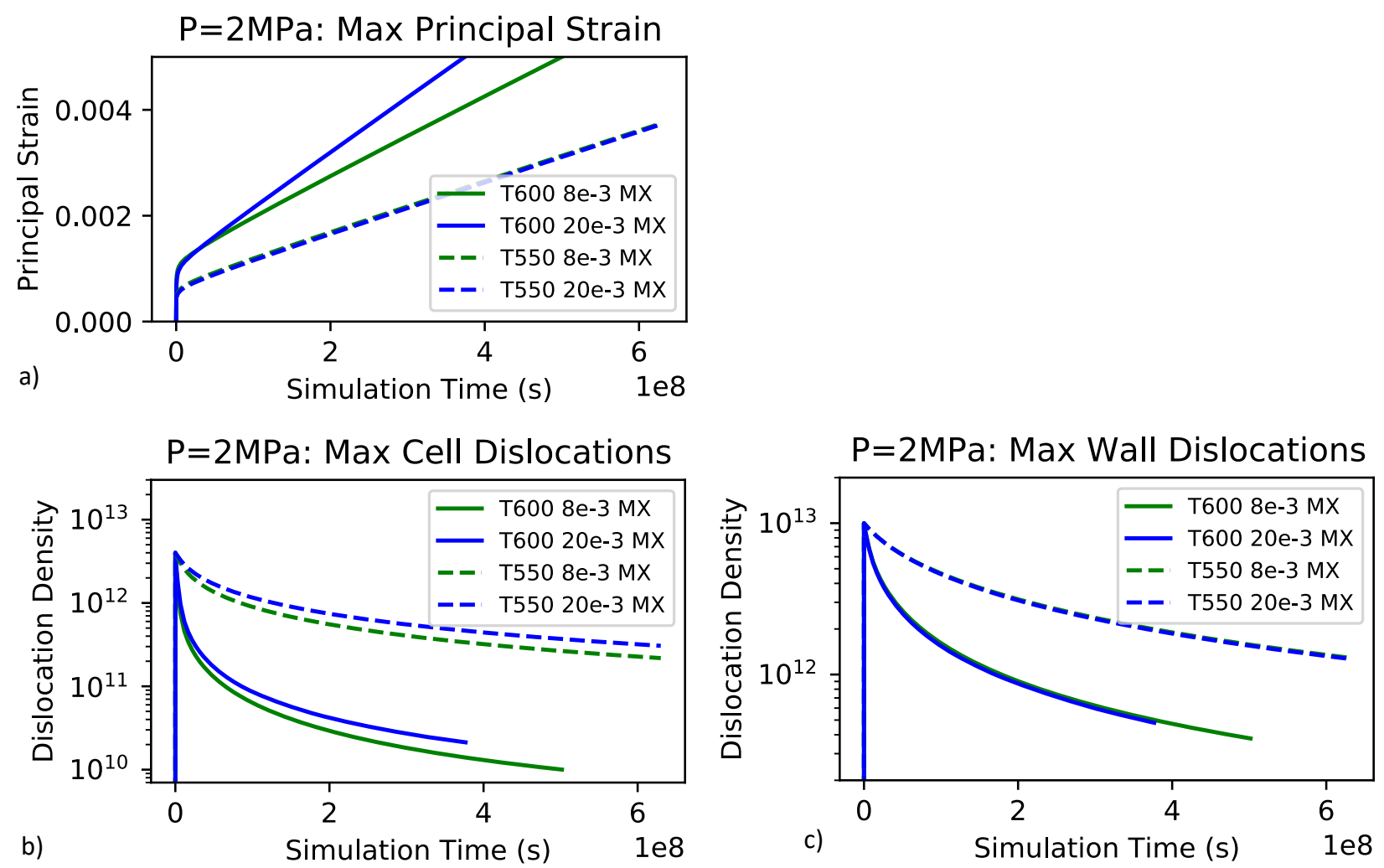

Figure 4.20: Maximum principal strain and dislocation density evolution as a function of simulation time for two different temperatures and $\mathrm{MX}$ phase content. Both simulations at $\mathrm{T}=600^{\circ}$ reach the critical principal strain of $0.5 \%$ prior to 20 years for $\mathrm{P}=2 \mathrm{MPa}$. Plotted values are the maximum elemental values found anywhere in the simulation at a particular time step. 


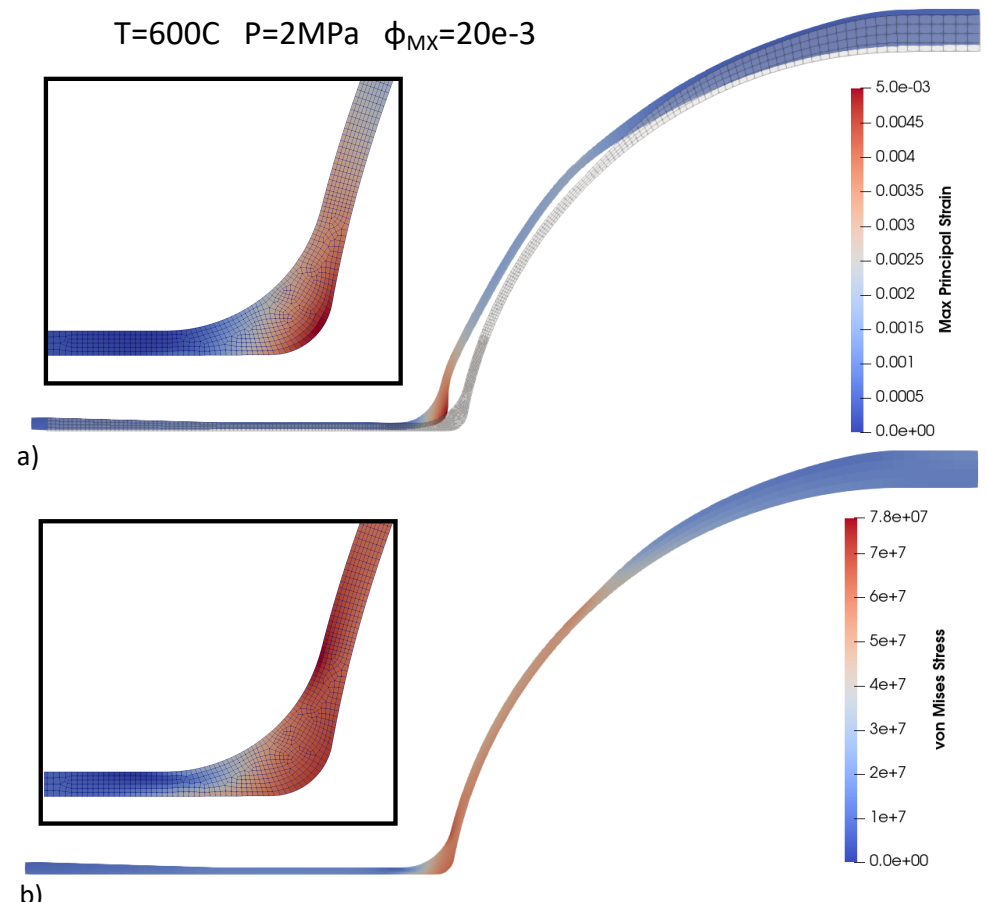

Figure 4.21: Constant pressure simulation results at $2 \mathrm{MPa}$ and $600{ }^{\circ} \mathrm{C}$ for LAROMANCE Grade 91 for mean dislocation density inputs (wall $=12 \times 10^{12} \mathrm{~m}^{-2}$ cell $=4 \times 10^{12} \mathrm{~m}^{-2}$ ) from Table 4.3 and a high MX phase content $\left(\phi_{M X}=20 \times 10^{-3}\right)$. The results shown are for a simulation time of 11.9 years at which point the maximum principal strain in one of the elements reached $0.5 \%$. (a) Final maximum principal strain contours on $20 \times$ displaced mesh, compared to the undeformed mesh in gray. (b) Final von Mises stress contours. Inset shows a close up of the transition region. 


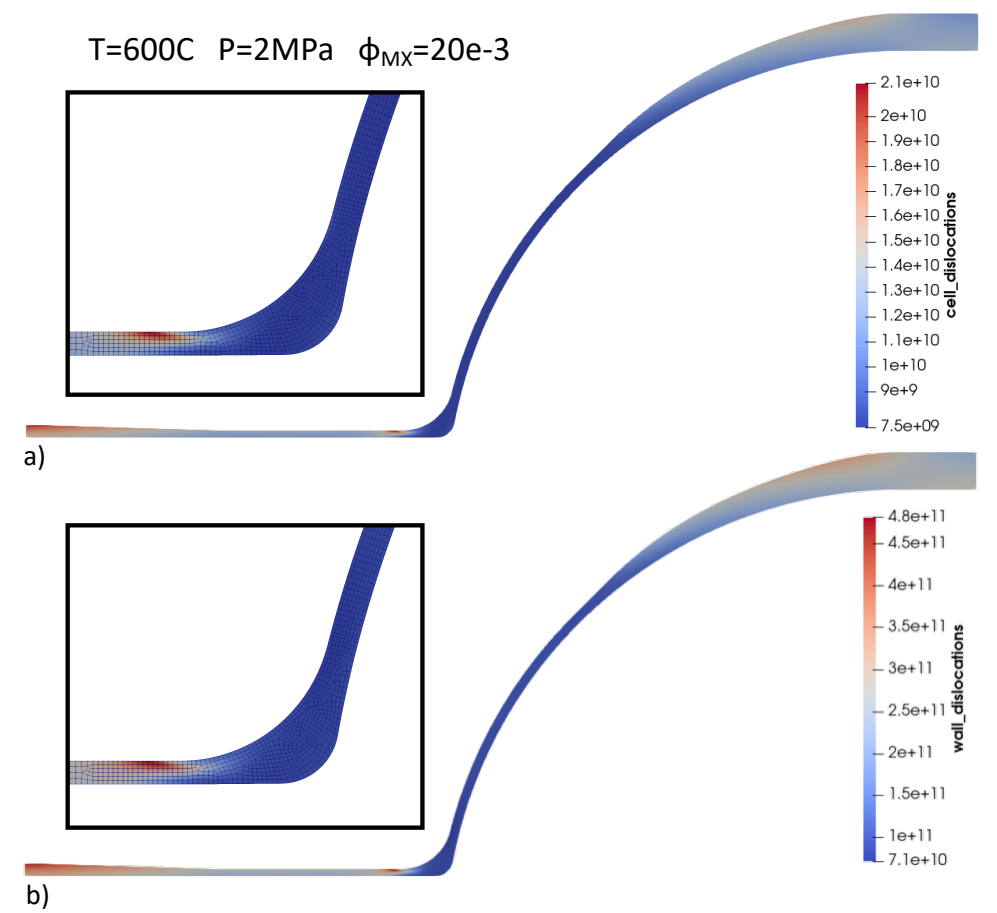

Figure 4.22: Constant pressure simulation results at $2 \mathrm{MPa}$ and $600{ }^{\circ} \mathrm{C}$ for LAROMANCE Grade 91 with mean values for the initial dislocation density inputs (wall $=12 \times 10^{-12} \mathrm{~m}^{-2}$ cell $=4 \times 10^{-12} \mathrm{~m}^{-2}$ ) from Table 4.3 and a high MX phase content $\left(\phi_{M X}=20 \times 10^{-3}\right)$. The results shown are for a simulation time of 11.9 years at which point the maximum principal strain in one of the elements reached $0.5 \%$. (a) Cell and (b) wall final dislocation density contours. Inset shows a close up of the transition region. 
while at $3 \mathrm{MPa}$ we see $100 \%$ of samples reach the critical strain. We see a similar trend for $\mathrm{T}=600{ }^{\circ} \mathrm{C}$, where no samples fail at $1.5 \mathrm{MPa}$ and $100 \%$ of the samples fail with a $0.5 \mathrm{MPa}$ increase in pressure.

Final Maximum Principal Strain Distributions at 20 Years $(\mathrm{T}=550 \mathrm{C})$

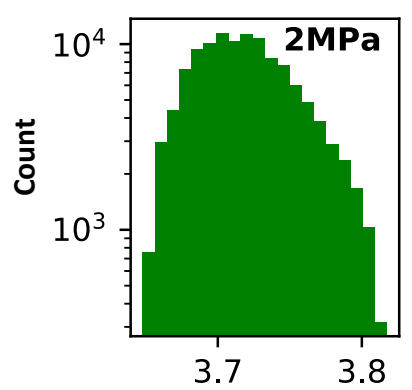

a) Principal Strain $1 e-3$

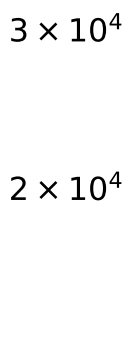

5.000

Principal Strain $1 \mathrm{e}-3$
Final Maximum Principal Strain Distributions at 20 Years $(\mathrm{T}=600 \mathrm{C})$

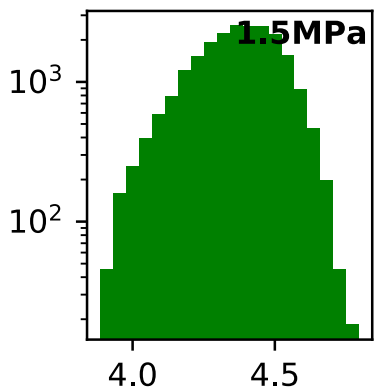

b) Principal Strain $1 \mathrm{e}-3$

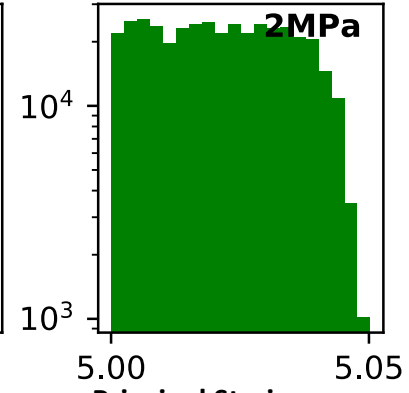

Principal Strain $1 \mathrm{e}-3$

Figure 4.23: Final maximum principal strain reached after 20 years for 4800 Monte Carlo samples of the material distributions with in the range of properties the LAROMANCE model was fit. (a) Two pressures at $\mathrm{T}=550^{\circ} \mathrm{C}(\mathrm{b})$ Two pressures at $\mathrm{T}=600^{\circ} \mathrm{C}$

In Figure 4.24 we plot the probability density function and cumulative distribution functions for the two samples that show $100 \%$ failure in Figure 4.23 . For $\mathrm{T}=550{ }^{\circ} \mathrm{C}$, there is a very tight distribution on the failure time, with all 4800 samples failing within about a year of each other at about 16 years. At $\mathrm{T}=600{ }^{\circ} \mathrm{C}$, the distribution of failure time is broader. Some samples begin failing after 10 years and all of the samples have failed in 20 years. These narrow failure distributions and narrow windows of pressures where failure goes from 0 to $100 \%$ were not observed in the pipe model in the previous section.
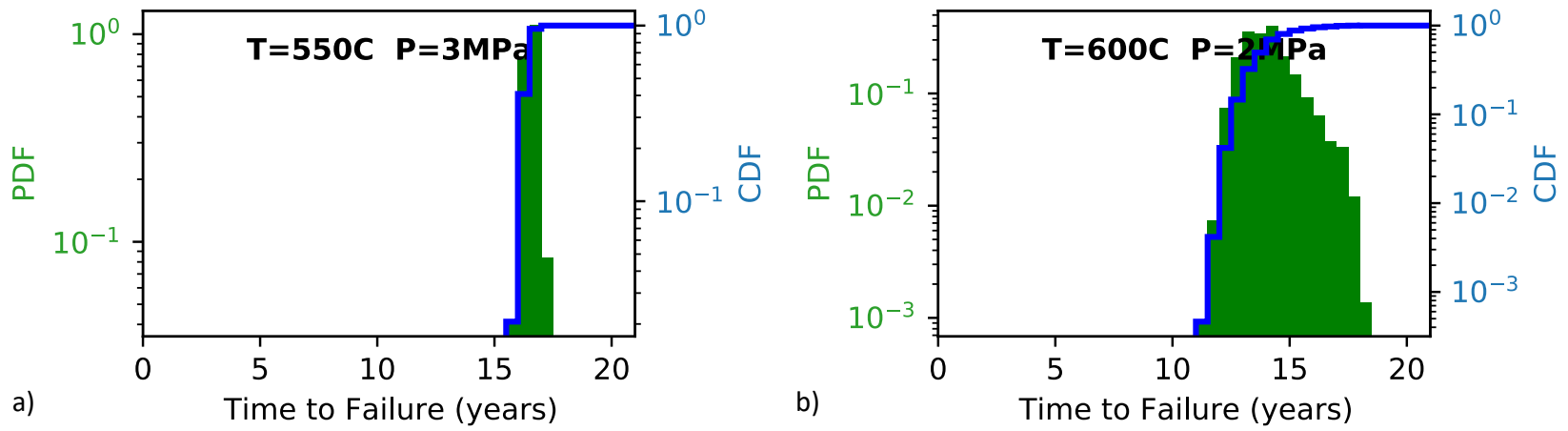

Figure 4.24: Probability density function (green histogram) and cumulative distribution functions for simulations in Figure 4.23 that reach a critical maximum principal strain of $0.5 \%$. (a) $\mathrm{T}=550{ }^{\circ} \mathrm{C}$, $\mathrm{P}=3 \mathrm{MPa}$, (b) $\mathrm{T}=600{ }^{\circ} \mathrm{C}, \mathrm{P}=2 \mathrm{MPa}$

We find that all of the samples reach more than $0.5 \%$ strain in 20 years. To establish correlations between microstructure and deformation kinetics we adopt an alternate criterion for differentiating the samples that are more likely to fail first. To do this, we consider the amount of time for the sample to reach the critical strain of $0.5 \%$. For this criterion, we find the first $1 \%$ of samples to fail for the pressures and temperature shown in Figure 4.24. We have plotted the material distributions leading to the earliest $1 \%$ of failure times in gold and compared this material distribution to the original distribution of material properties in green. It is interesting to note that although this is the first observation of failure for $\mathrm{T}=550{ }^{\circ} \mathrm{C}$, the material properties leading to the critical strain measure are similar to those of the pipe model for $\mathrm{T}=550{ }^{\circ} \mathrm{C}$ in Figure 4.15 . We 
see $\phi_{M X}$ has a negligible affect while a low initial wall dislocation density seems to be most closely correlated with earliest failure times. At the higher temperature of $\mathrm{T}=600^{\circ} \mathrm{C}$, we again see high $\phi_{M X}$ leading to higher strains.

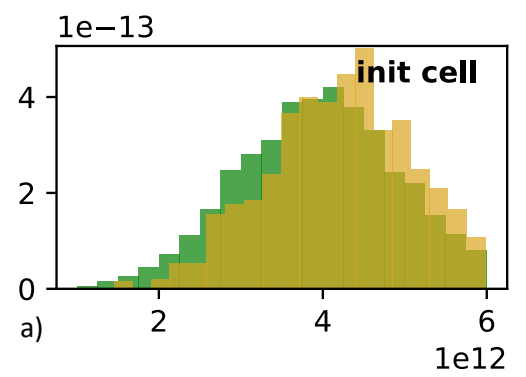

Input Parameters ( $\mathrm{T}=550 \mathrm{C}, \mathrm{P}=3 \mathrm{MPa})$
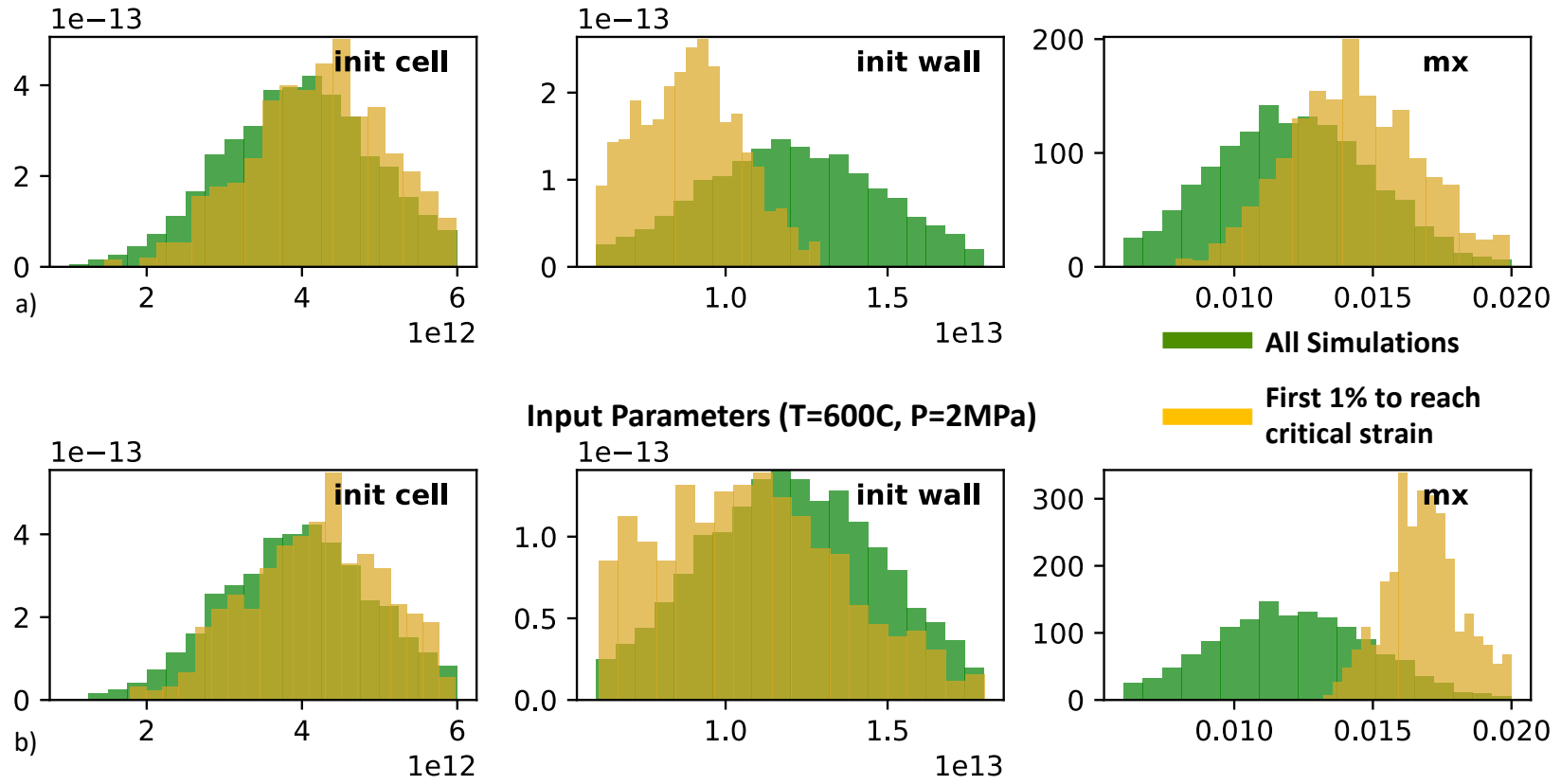

Figure 4.25: Normalized histograms of input material parameters for all 4800 samples in green and the earliest $1 \%$ of simulations to reach the critical strain of $0.5 \%$ in Figure 4.24 shown in gold. (a) $\mathrm{T}=550{ }^{\circ} \mathrm{C}, \mathrm{P}=3 \mathrm{MPa}$. (b) $\mathrm{T}=600{ }^{\circ} \mathrm{C}, \mathrm{P}=2 \mathrm{MPa}$

The evolution of dislocations in Figure 4.26 is similar to that observed previously for the pipe model and shown in Figures 4.15 and 4.16. At $\mathrm{T}=550{ }^{\circ} \mathrm{C}$, the lower initial wall dislocation density continues to evolve to a lower than average final state. At $\mathrm{T}=600{ }^{\circ} \mathrm{C}$, we again see a lower final cell dislocation density, and it is unclear if the lower cell dislocation density is correlated with the higher $\phi_{M X}$ or with failure.

$\mathrm{T}=550 \mathrm{C} . \mathrm{P}=3 \mathrm{MPa}$

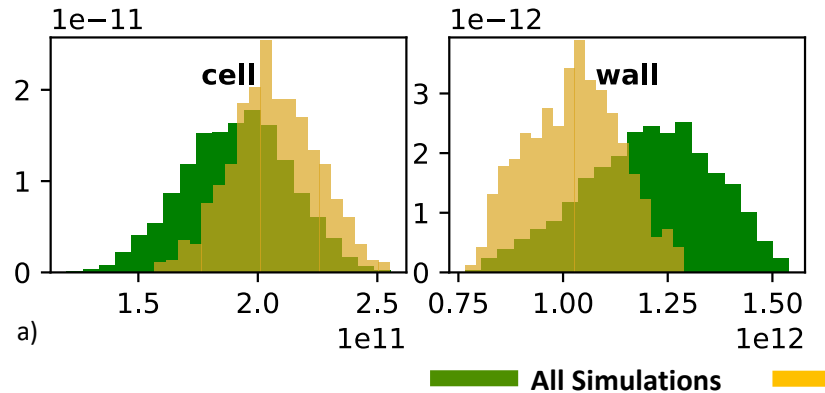

$\mathrm{T}=600 \mathrm{C} . \mathrm{P}=2 \mathrm{MPa}$

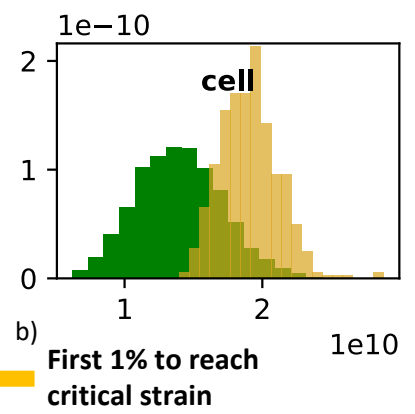

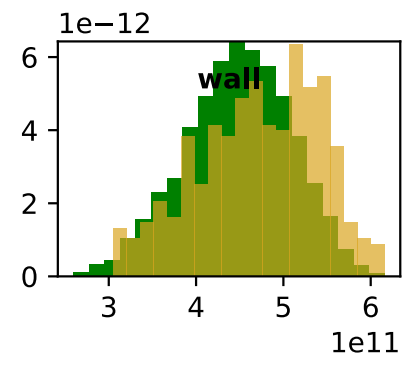

Figure 4.26: Normalized histograms of the final dislocation densities for all 4800 samples in green and the earliest $1 \%$ of simulations to reach the critical strain of $0.5 \%$ in Figure 4.24 shown in gold. 


\subsection{Discussion}

We presented a statistical characterization of the NEML and LAROMANCE Grade 91 material models over their ranges of applicability in two fairly straightforward applications. We performed these analyses for a pressure range between $1-8 \mathrm{MPa}$ and two temperatures, $550{ }^{\circ} \mathrm{C}$ and $600{ }^{\circ} \mathrm{C}$. The simplified pipe cross section model provided a fast-running model that allowed us to test the bounds of the material distributions being sampled and boundary conditions being applied. It also provided a rapid assessment of the sensitivity of the strain to certain material inputs. The fast-running model uncovered the same material sensitivities as those found in the more complicated sphere nozzle model. The more complicated sphere nozzle model provided a test of robustness for both material models under realistic conditions of interest for engineering applications, with more complex geometry, more refined meshes, and stress gradients in the nozzle transition region. The two models robustly ran for over a million simulations for a range of input parameters sampled over their fitted distributions.

We note that the NEML Grade 91 material model is a phenomenological model fit to macroscopic experimental data with a large variation in input parameters resulting in a large range of measured strains at the end of the 20 year simulation. The LAROMANCE Grade 91 model is a reduced order model built up from mesoscale simulations fit to microstructural data and resulted in a much smaller range of measured strains over the twenty year simulation. These two Grade 91 material models are quantifying very different effects. The NEML model predicted larger strain ranges due to the uncertainty in the model parameters as they were fit to available experimental data. The strain range calculated by the LAROMANCE model is due the variability in the initial microstructure and not on phenomenological model parameters.

Future component level modeling work should include:

- Importance sampling of statistical data to reduce the number of Monte Carlo samples required to achieve statistically relevant failure probabilities. This will be important for cyclic loading scenarios in both the NEML and LAROMANCE models.

- Variability of microstructure of the component model and its effect on the strain range calculated by the LAROMANCE model.

- Reduced order and surrogate models of component-level response to reduce computational complexity and simulation time. 


\section{Bibliography}

[1] A. Casagranda, S. A. Pitts, B. W. Spencer, A. Chakraborty, M. C. Messner, and L. Capolungo. Evaluation of high temperature material models for high temperature advanced reactor component analysis. In Proceedings of ASME 2020 Pressure Vessels and Piping Conference, Paper No. 21614, Minneapolis, Minnesota, July 2020.

[2] M. C. Chaturvedi and Y. Han. Effect of particle size on the creep rate of superalloy Inconel 718. Materials Science and Engineering, 89(89):L7-L10, 1987.

[3] W. C. Comeli, A. D. S. Rocha, C. A. S. D. Oliveira, G. Lemos, and R. D. M. Castro. Effects of tempering temperature on the microstructure and creep resistance of X22CrMoV12-1 steel used on steam turbine blades. Journal of Materials Science, 8(4):65-72, 2018.

[4] H. Han, J. Shen, and J. Xie. Effects of Precipitates Evolution on Low Stress Creep Properties in P92 Heat-resistant Steel. Scientific Reports, 8(1):1-10, 2018. 


\section{Summary and Future Work}

This report summarizes the development and calibration of two complementary constitutive modeling approaches for the viscoplastic response of Grade 91 alloy in high temperature environments prototypical of those that would be experienced in structural components of advanced reactors.

- Development of uncertain parameters for phenomenological NEML models (ANL) A Bayesian approach was developed and used to derive distributions of uncertain parameters for a classical model implemented in the NEML library based on a data set that included a wide range of treatments of Grade 91 alloy.

- Development and parameterization of a reduced order (LAROMANCE) model (LANL) A reduced order model suitable for engineering-scale analysis that is based on the results of a large set of mesoscale simulations of a subset of experimental data for a specific treatment of Grade 91 alloy. The underlying mesoscale models allow the characteristics of a particular alloy to be taken into account, while representing their response using a reduce order model allows for practical engineering-scale simulations with reasonable computational expense.

- Stochastic engineering-scale simulations of component lifetime (INL) Both the NEML and LAROMANCE models have been integrated into the Grizzly code, and used for proof-of-concept uncertainty quantification analyses of a simple component under prototypical conditions. The built-in stochastic analysis capabilities in the MOOSE framework are used here to run large sets of simulations for this uncertainty quantification analysis.

As would be expected, because the reduced order models are developed for a much more tightly defined alloy, they predict tighter distributions of the time to failure than the phenomenological models, which are calibrated to a broader set of data. Also important is that these simulations demonstrate that a reduced order modeling approach can be successfully deployed to propagate uncertainties from the material scale to practical engineering-scale component simulations.

Details of logical follow-on work have been provided in the individual sections of this report where they were discussed. These tasks are summarized at a high level here:

- The NEML Grade 91 material model requires improvements for cyclic loading. These improvements should include an improved representation of kinematic hardening in the plasticity models for cyclic loading. The Bayesian MCMC method should also be improved to better fit experimental scatter.

- The LAROMANCE Grade 91 material models will provide a better estimate of the structure's variability if the component geometry is stochastically seeded with randomize material properties.

- The LAROMANCE model does not yet include tertiary creep and damage. By basing the material response on a spatially resolved microstructure model that includes these effects, this model can be expanded to include those effects.

- This work has demonstrated the applicability of the LAROMANCE model under relatively narrow conditions. The robustness of this constitutive modeling approach under a broader range of conditions can be improved with further development. 
- Further development of the stochastic sampling tools used in Grizzly, including the use of more sophisticated adaptive importance sampling, will permit accurate sampling in the tails of the distributions with lower computational resource demands.

- The engineering scale analysis tools need further development to include the effects of damage.

- More realism is needed in the engineering-scale component models and in the failure criteria. This study has demonstrated a reasonable proof of concept, but models that push the constitutive models into more numerically challenging regimes will better demonstrate the robustness of this approach on more representative real-life problems. 


\section{Acknowledgments}

The submitted manuscript has been authored by a contractor of the U.S. Government under Contract DEAC07-05ID14517. Accordingly, the U.S. Government retains a non-exclusive, royalty free license to publish or reproduce the published form of this contribution, or allow others to do so, for U.S. Government purposes.

The work of Messner and Chakraborty was sponsored by the U.S.Department of Energy, under Contract No. DEAC02-06CH11357 with Argonne National Laboratory, managed and operated by UChicago Argonne LLC.

This research made use of the resources of the High Performance Computing Center at Idaho National Laboratory, which is supported by the Office of Nuclear Energy of the U.S. Department of Energy and the Nuclear Science User Facilities under Contract No. DE-AC07-05ID14517. 